\title{
Effects of Hazelnut Consumption on Cardiometabolic Risk Factors and Acceptance: A Systematic Review
}

\author{
Rachel Brown (D, Lara Ware and Siew Ling Tey *(D) \\ Department of Human Nutrition, University of Otago, Dunedin 9054, New Zealand; \\ rachel.brown@otago.ac.nz (R.B.); lara.ware@otago.ac.nz (L.W.) \\ * Correspondence: siewling.tey@otago.ac.nz
}

check for updates

Citation: Brown, R.; Ware, L.; Tey, S.L. Effects of Hazelnut Consumption on Cardiometabolic Risk Factors and Acceptance: A Systematic Review. Int. J. Environ. Res. Public Health 2022, 19, 2880. https://doi.org/10.3390/ ijerph19052880

Academic Editor: Lorrene D. Ritchie

Received: 30 November 2021

Accepted: 12 February 2022

Published: 1 March 2022

Publisher's Note: MDPI stays neutral with regard to jurisdictional claims in published maps and institutional affiliations.

Copyright: (C) 2022 by the authors. Licensee MDPI, Basel, Switzerland. This article is an open access article distributed under the terms and conditions of the Creative Commons Attribution (CC BY) license (https:// creativecommons.org/licenses/by/ $4.0 /)$.

\begin{abstract}
Despite being rich sources of monounsaturated fat and a number of vitamins, minerals, and phytonutrients, hazelnuts have received less attention than some other nut types. A qualitative systematic review was carried out to determine the effects of hazelnut consumption on acceptance and markers of cardiometabolic health, including blood lipids and lipoproteins, apolipoproteins A1 and B100, body weight and composition, blood pressure, glycemia, antioxidant status, oxidative stress, inflammation, and endothelial function. In total, 22 intervention studies (25 publications) met our inclusion criteria. The findings indicate some improvements in cardiometabolic risk factors; however, limitations in study design mean interpretation is problematic. The inclusion of hazelnuts in the diet did not adversely affect body weight and composition. Acceptance of hazelnuts remained stable over time confirming nut consumption guidelines are feasible and sustainable. Future studies using more robust study designs in a variety of populations are required to draw more definitive conclusions on the health benefits of hazelnut consumption.
\end{abstract}

Keywords: hazelnuts; blood lipids and lipoproteins; apolipoproteins; body weight and composition; blood pressure; glycaemia; oxidative stress; inflammation; endothelial function; acceptance

\section{Introduction}

Observations from large cohort studies indicate regular nut consumption is associated with a reduction in the risk of total mortality and a number of chronic diseases, such as cardiovascular disease and certain cancers [1-3]. Studies on diabetes, hypertension, and stroke are equivocal, with the majority showing no significant associations [4-7]. Although nuts are high in energy and fat, observational studies report that nut consumers are leaner than non-nut consumers [8,9]. Additionally, longitudinal studies report nut consumption is associated with a lower risk of overweight and obesity, weight gain, and deposition of abdominal adiposity [10,11].

Randomised controlled trials have shown improvements in risk factors of chronic disease with regular nut consumption. For example, total cholesterol and low-density lipoprotein cholesterol (LDL-C) are consistently lowered by regular nut consumption, with reductions more pronounced in those with elevated cholesterol concentrations [12-14]. Findings on blood pressure and biomarkers of oxidation, inflammation, and endothelial function are mixed, with some showing positive effects, while others report no effect [13,15-18]. In support of observational studies, intervention studies have found that adding nuts to the usual diet results in no weight gain or less than expected weight gain given the additional calories provided by nuts [11,19].

Despite being the second-largest nut produced worldwide, hazelnuts have received less attention regarding their health benefits than some other nut types [20,21]. Hazelnuts are high in monounsaturated fats and are a source of fibre, vitamin E, folate, potassium, copper, manganese, phosphorous, magnesium, and phytosterols [21]. They also contain high amounts of flavonoids and phenolic compounds, especially in their skin [22,23]. 
While there are many recent comprehensive systematic reviews and meta-analyses on the health effects of almonds [24], cashews [25], pistachios [26,27], and walnuts [28-30], only one systematic review and meta-analysis has reviewed the evidence on hazelnuts. This review published in 2016 reported the effects of hazelnut consumption on blood lipids and body weight [31]. Only three of the nine studies included in this review were randomised controlled trials. The meta-analysis of these three studies showed a significant reduction in LDL-C and a tendency for a reduction in total cholesterol, but no significant changes in high-density lipoprotein cholesterol (HDL-C), triglycerides (TAG), or body mass index (BMI).

We aimed to extend this review to include studies that have been published since its publication and to expand the outcomes to also include apolipoproteins, blood pressure, glycaemic response, acceptance, and markers of inflammation, oxidation, and endothelial function.

\section{Materials and Methods}

\subsection{Search Strategy}

The protocol for this systematic review was registered with PROSPERO (registration number CRD42020203171). Medline (via Ovid), PubMed, Scopus, and Google Scholar databases were searched on 29 July 2020. The search was updated on 28 November 2021, but no further studies meeting our eligibility criteria were identified. The search strategy was limited to human studies and articles written in the English language. Reference lists from publications identified by our searches were manually searched to identify relevant research not found in the database searches. Search terms are outlined in Supplementary Material Table S1.

Study selection was then conducted by SLT and RB using Rayyan [32], and any disagreements were resolved by consultation.

\subsection{Inclusion and Exclusion Criteria}

Studies were included if they met the following criteria: were intervention studies in human participants, included hazelnuts, and evaluated at least one of the study outcomes (see Table 1). Studies were excluded if they were non-English language, reviews, expert opinions, theses, animal, or in vitro studies, if the independent effects of hazelnuts could not be assessed, or if hazelnut oil was used as the test food. Our PICOS statement is outlined in Table 1.

Table 1. PICOS criteria for inclusion and exclusion of studies.

\begin{tabular}{cl}
\hline Parameter & Criterion \\
\hline Participants & Humans \\
\hline Intervention & Consumption of hazelnuts \\
\hline Comparator & No nut control, control food, baseline \\
\hline Outcomes & $\begin{array}{l}\text { Total cholesterol, LDL-C, HDL-C, TAG, apolipoprotein A1, apolipoprotein B100, } \\
\text { body weight, blood pressure, glycaemic control, antioxidant status, vitamin E, } \\
\text { oxidative stress, inflammatory markers, endothelial function, acceptance }\end{array}$ \\
\hline Study design & $\begin{array}{l}\text { Intervention studies in peer-reviewed journals where hazelnuts were the dietary } \\
\text { component under study. }\end{array}$ \\
\hline
\end{tabular}

\subsection{Data Extraction}

Data extracted included authors, year, study design, participant characteristics, intervention period, treatments (including dose), and outcomes.

\subsection{Study Quality}

This review was undertaken using the principles outlined in the PRISMA 2020 statement [33]. The risk of bias for each study was assessed by all authors using the Cochrane Collaboration Risk of Bias Tool for randomised controlled intervention studies [34] and the 
Risk of Bias in Non-Randomised Studies—of Interventions (ROBINS-I) for non-randomised intervention studies [35].

The Cochrane Collaboration Risk of Bias Tool considers the following domains: selection bias, reporting bias, performance bias, detection bias, attrition bias, and any other identified biases [34]. Each domain was classified as low, high, or unclear risk of bias. Studies with low risk for $\leq$ one domain were classified as poor, studies with a low risk of bias for two domains were classified as fair, and studies with a low risk of bias in at least three domains were classified as good.

The ROBINS-I tool considers bias in the following domains: confounding, selection of study participants, classification of interventions, deviations from intended interventions, missing data, measurement of outcomes, selection of reported results [35]. Each domain was classified as low, moderate, serious, or critical. Studies for low risk of bias for all domains were classified as low, studies with low or moderate risk of bias for all domains were classified as moderate, studies with serious risk of bias in at least one domain, but not at critical risk of bias in any domain were classified as serious, and studies with critical risk of bias in at least one domain were classified as critical.

\section{Results}

The search criteria returned a total of 787 articles. A total of 475 were excluded as duplicates. After abstract review, 58 were included for review. After retrieval of the selected papers, 25 papers (22 studies) were included in the present review (Figure 1). Seven of the studies were conducted in New Zealand, seven in Italy, six in Turkey, one in Iran, and one in the USA.

\subsection{Risk of Bias}

The quality of the methods for the studies is presented in Tables 2 and 3. Overall, 10 randomised trials were rated as good, one as fair, and one as poor (Table 2). For nonrandomised trials, the overall risk of bias for two studies was rated as moderate, and eight were rated as critical (Table 3 ). 


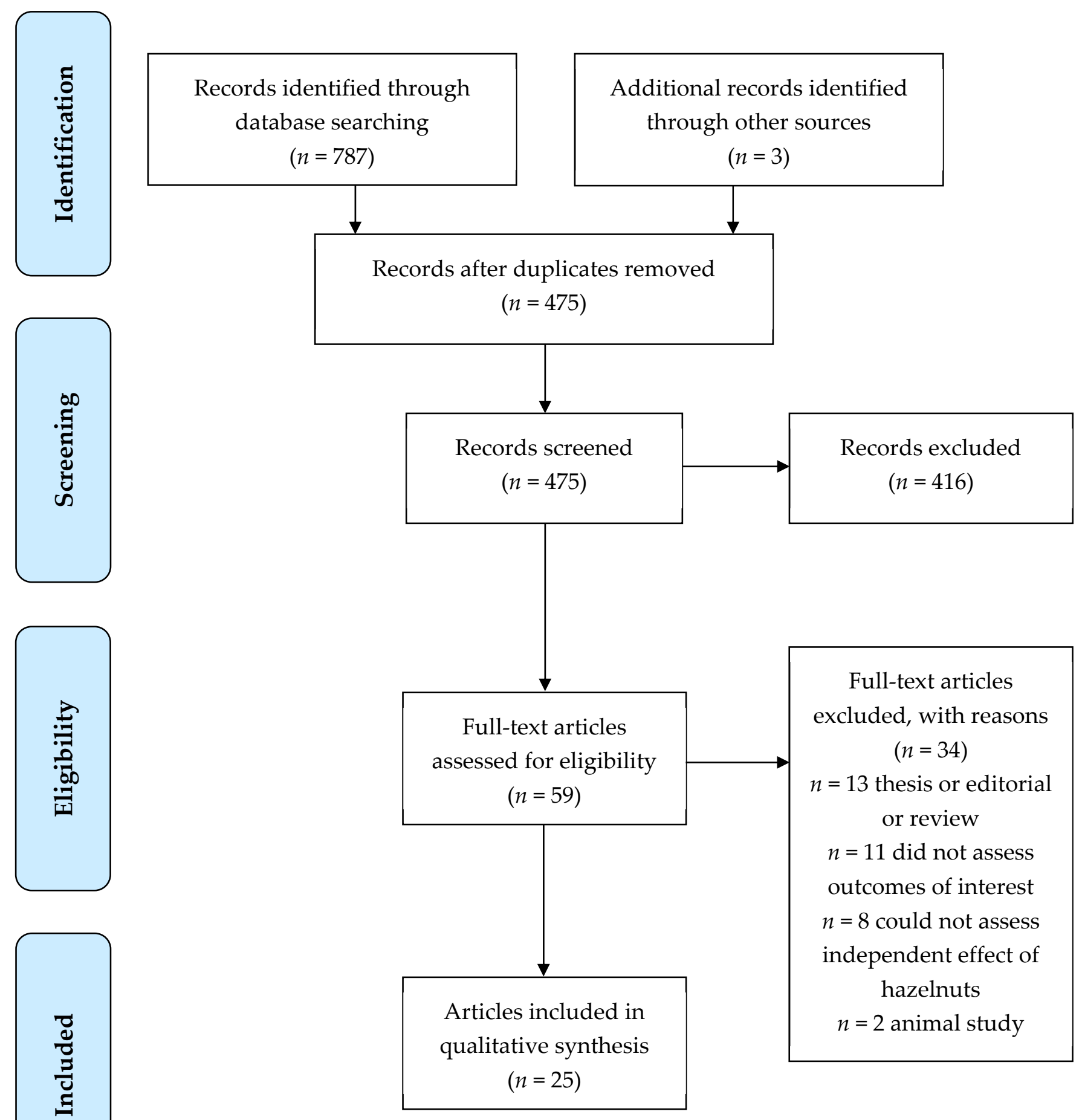

Figure 1. Flow diagram of the literature search process. 
Table 2. Study quality and risk of bias for randomised trials $(n=12)^{1}$.

\begin{tabular}{|c|c|c|c|c|c|c|c|}
\hline $\begin{array}{c}\text { Author, Year } \\
\text { (Study Location) }\end{array}$ & $\begin{array}{c}\text { Random Sequence } \\
\text { Generation }\end{array}$ & $\begin{array}{c}\text { Allocation } \\
\text { Concealment }\end{array}$ & Selective Reporting & Blinding & $\begin{array}{c}\text { Blinding of Outcome } \\
\text { Assessment }\end{array}$ & $\begin{array}{l}\text { Incomplete Outcome } \\
\text { Assessment }\end{array}$ & Overall Quality \\
\hline Adamo et al., 2018 [36] (Italy) & low & unclear & high & high & high & low & poor \\
\hline Damavandi et al., 2012 [37] (Iran) & low & unclear & low & high & low & low & good \\
\hline Damavandi et al., 2013 [38] (Iran) & low & unclear & low & high & low & low & good \\
\hline Devi et al., 2016 [40] (New Zealand) & low & low & low & high & low & low & good \\
\hline Di Renzo et al., 2017 [41] (Italy) & low & low & low & high & low & low & good \\
\hline Guaraldi et al., 2018 [42] (Italy) & low & low & low & high & low & low & good \\
\hline Tey et al., 2011 [43] (New Zealand) & low & low & low & high & low & low & good \\
\hline Tey et al., 2011 [44] (New Zealand) & low & low & low & high & low & low & good \\
\hline Tey et al., 2012 [46] (New Zealand) & low & low & low & high & low & low & good \\
\hline Tey et al., 2013 [47] (New Zealand) & low & low & low & high & low & low & good \\
\hline Tey et al., 2015 [48] (New Zealand) & low & low & low & high & low & low & good \\
\hline Tey et al., 2017 [49] (New Zealand) & low & low & low & high & low & low & good \\
\hline Yilmaz et al., 2019 [50] (Turkey) & unclear & unclear & low & high & high & low & fair \\
\hline
\end{tabular}

${ }^{1}$ Overall quality: good (low risk of bias in at least three domains), fair (low risk of bias in at least two domains), poor (low risk of bias in one or less domain). There were three studies with two publications, each reporting different study outcomes, i.e., the first study [37,38], the second study [43,44], and the third study [45,46].

Table 3. Study quality and risk of bias for non-randomised trials $(n=10)^{1}$.

\begin{tabular}{|c|c|c|c|c|c|c|c|c|}
\hline $\begin{array}{c}\text { Author, Year } \\
\text { (Study Location) }\end{array}$ & $\begin{array}{l}\text { Bias Due to } \\
\text { Confounding }\end{array}$ & $\begin{array}{l}\text { Bias in Selection } \\
\text { of Participants } \\
\text { into the Study }\end{array}$ & $\begin{array}{l}\text { Bias in Classification } \\
\text { of Interventions }\end{array}$ & $\begin{array}{l}\text { Bias Due to Deviations } \\
\text { from Intended } \\
\text { Interventions }\end{array}$ & $\begin{array}{c}\text { Bias Due to } \\
\text { Missing Data }\end{array}$ & $\begin{array}{l}\text { Bias in Measurement } \\
\text { of Outcomes }\end{array}$ & $\begin{array}{l}\text { Bias in Selection of the } \\
\text { Reported Result }\end{array}$ & Overall Risk of Bias \\
\hline Alphan et al. 1997 [51] (Turkev) & critical & no information & low & low & no information & moderate & serious & critical \\
\hline Di Renzo et al., 2014 [52] (Italy) & critical & low & low & low & moderate & moderate & serious & critical \\
\hline Di Renzo et al., 2019 [53] (Italy) & critical & low & low & low & moderate & moderate & low & critical \\
\hline Durak et al., 1999 [54] (Turkey) & critical & moderate & low & low & no information & serious & serious & critical \\
\hline Mercanligil et al., 2007 [55] (Turkey) & critical & low & low & low & low & serious & low & critical \\
\hline $\begin{array}{l}\text { Michels et al., } 2018 \text { [56] (USA) } \\
\text { (USA) }\end{array}$ & critical & moderate & low & low & moderate & moderate & low & critical \\
\hline Orem et al., 2013 [57] (Turkey) & serious & moderate & low & low & no information & moderate & moderate & moderate \\
\hline Santi et al., 2017 [58] (Italy) & serious & moderate & low & low & no information & moderate & moderate & moderate \\
\hline Tey et al., 2015 [59] (New Zealand) & critical & low & low & low & moderate & moderate & low & critical \\
\hline Yucesan et al., 2010 [60] (Turkey) & critical & moderate & low & low & no information & moderate & moderate & critical \\
\hline
\end{tabular}

${ }^{1}$ Overall risk of bias judgement: low (low risk of bias for all domains), moderate (low or moderate risk of bias for all domains), serious (serious risk of bias in at least one domain, but not at critical risk of bias in any domain), critical (critical risk of bias in at least one domain). 


\subsection{Blood Lipids and Lipoproteins}

In total, 17 studies examined the effects of hazelnut consumption on blood lipids and lipoproteins (Table 4). Different study designs included: randomised parallel $(n=6)$, randomised crossover $(n=2)$, sequential $(n=2)$, double control sandwich $(n=2)$, and single intervention $(n=5)$. Interventions ranged in duration from 2 to 16 weeks. Sample sizes ranged from 15 to 118 and were heterogeneous in nature. For example, nine samples comprised healthy participants, five included those with hyperlipidaemia (including one with children), two included those with type 2 diabetes, and one specifically recruited people with overweight or obesity.

Nine studies compared hazelnut consumption to a no-nut control. Of these, two reported significantly lower total cholesterol and LDL-C $[57,58]$, and three reported significantly higher HDL cholesterol $[36,38,57]$ in the hazelnut group compared to the control. Only one study reported a significant reduction in TAG in the hazelnut group compared to the control [57]. Four studies reported no significant differences in any of the outcomes $[39,45,47,50]$.

Two studies included a hazelnut and high carbohydrate treatment. Alphan et al. [51] reported significant decreases in total and LDL-C in the hazelnut group, with significant increases in LDL-C in the high carbohydrate group. However, they failed to report between-group differences. Mercanligil et al. [55] reported significantly higher HDL-C in the hazelnut group compared to the high carbohydrate control.

Of the single intervention studies, three observed reductions in total cholesterol $[53,54,60]$, four in LDL-C [53,54,56,60], while one reported an increase in HDL-C [54], and one an increase in TAG [54].

Two randomised crossover studies compared different forms of hazelnuts. One study compared ground vs. sliced vs. whole nuts [43]. There were no significant differences between treatments, but all three forms were associated with significant reductions in total cholesterol and LDL-C, and significant increases in HDL-C. A further study compared raw vs. roasted hazelnuts [49]. HDL-C was significantly higher following raw hazelnuts, while TAG was significantly lower following the roasted hazelnuts. There were no between-group differences for total cholesterol and LDL-C. Within-group, changes included a significant decrease in LDL-C and a significant increase in HDL-C with raw hazelnut consumption.

Overall, 9 (4 RCTs, 3 single arm, 2 different forms) and 10 (4 RCTs, 4 single arm, 2 different forms) of the 16 studies reported statistically significant reductions in total and LDL-C with hazelnut consumption, respectively. For HDL-C, 7 (4 RCTs, 1 single intervention, 2 different forms) studies reported statistically significant increases. Two (1 RCT, 1 different forms) studies reported significant reductions in TAG while 1 (single intervention) reported a significant increase.

\subsection{Apolipoproteins $A$ and $B$}

Eight studies examined the effects of hazelnut consumption on apolipoproteins (apo) A and B (Table 5). Study design included randomised parallel $(n=1)$, randomised crossover $(n=2)$, sequential $(n=2)$, double control sandwich $(n=1)$, and single intervention $(n=2)$. Most of the studies were 4 weeks in duration, with one being 2 weeks and one 12 weeks. Sample sizes ranged from 15 to 107 participants. Three studies included healthy participants, three included those with hyperlipidaemia (including mild hyperlipidaemia), one included those with type 2 diabetes, and one specifically recruited people with overweight or obesity. 
Table 4. Effects of hazelnut consumption on blood lipids and lipoproteins ( $n=17$ )

\begin{tabular}{|c|c|c|c|c|c|c|c|c|c|}
\hline Author, Year & $\begin{array}{c}\text { Study } \\
\text { Design }\end{array}$ & $\begin{array}{c}\text { Participant } \\
\text { Characteristics }\end{array}$ & Duration & Treatment & $\begin{array}{c}\mathrm{TC} \\
\mathrm{mmol} / \mathrm{L}\end{array}$ & $\begin{array}{l}\mathrm{LDL}-\mathrm{C} \\
\mathrm{mmol} / \mathrm{L}\end{array}$ & $\begin{array}{l}\mathrm{HDL}-\mathrm{C} \\
\mathrm{mmol} / \mathrm{L}\end{array}$ & $\begin{array}{c}\mathrm{TAG} \\
\mathrm{mmol} / \mathrm{L}\end{array}$ & Between Treatments \\
\hline \multirow{10}{*}{ Adamo et al., 2018 [36] } & \multirow{10}{*}{$\begin{array}{l}\text { Randomised parallel } \\
6 \text { treatments }\end{array}$} & \multirow{10}{*}{$\begin{array}{c}61(31 \mathrm{M}, 30 \mathrm{~F}) \mathrm{BMI} \\
18.5-24.9 \mathrm{~kg} / \mathrm{m}^{2}\end{array}$} & \multirow{10}{*}{ 2-weeks } & $\begin{array}{c}\text { Breakfasts including: } \\
\text { (i) } 30 \mathrm{~g} / \mathrm{d} \text { peeled hazelnut paste }\end{array}$ & NR & NR & NR & NR & \multirow{10}{*}{$\begin{array}{l}30 \mathrm{~g} \text { of unpeeled hazelnut } \\
\text { significantly increased HDL-C } \\
\text { compared to control } \\
(16.0 \%, p=0.02)\end{array}$} \\
\hline & & & & Baseline & NR & 2.49 & 1.68 & NR & \\
\hline & & & & Change $^{1}$ & NR & $-0.16^{\mathrm{a}}$ & $+0.14^{\mathrm{b}}$ & NR & \\
\hline & & & & $\%$ change & $-2.0 \%$ & $-6.0 \%$ & $+16.0 \%$ & NR & \\
\hline & & & & $\begin{array}{c}\text { (iii) snack with } 30 \mathrm{~g} / \mathrm{d} \text { peeled hazelnut } \\
\text { paste }\end{array}$ & NR & NR & NR & NR & \\
\hline & & & & (iv) snack with $2.5 \mathrm{~g}$ cocoa powder & NR & NR & NR & NR & \\
\hline & & & & \multirow{3}{*}{$\begin{array}{c}\text { Baseline } \\
\text { (v) Snack with } 30 \mathrm{~g} / \mathrm{d} \text { peeled hazelnut } \\
\text { paste and } 2.5 \mathrm{~g} \text { cocoa powder } \\
\% \text { change }\end{array}$} & NR & NR & NR & NR & \\
\hline & & & & & NR & NR & NR & NR & \\
\hline & & & & & $-0.9 \%$ & $-3.4 \%$ a & $+5.2 \%$ & NR & \\
\hline & & & & $\begin{array}{l}\text { (vi) no snack control group } \\
\text { N.B. Data was only presented for } \\
\text { treatment ii vs. control and treatment v } \\
\text { vs. control }\end{array}$ & NR & NR & NR & NR & \\
\hline \multirow{5}{*}{ Alphan et al., 1997 [51] } & \multirow{5}{*}{$\begin{array}{c}\text { Sequential } \\
\text { intervention periods }\end{array}$} & \multirow{5}{*}{$\begin{array}{l}19(5 \mathrm{M}, 14 \mathrm{~F}) \text { with } \\
\text { type } 2 \text { diabetes }\end{array}$} & \multirow{5}{*}{30 days } & Baseline & 5.40 & 3.36 & 0.95 & 2.78 & \multirow{5}{*}{ Between-group analysis NR. } \\
\hline & & & & (i) High $\mathrm{CHO}$ diet (60\% CHO, $25 \%$ fat) & 5.67 & 3.92 & 0.97 & 2.45 & \\
\hline & & & & Change $^{1}$ & $+0.27^{\mathrm{b}}$ & $+0.56^{\mathrm{b}}$ & +0.02 & -0.33 & \\
\hline & & & & \multirow{2}{*}{$\begin{array}{c}\text { Baseline } \\
\text { (ii) Hazelnuts ( } 40 \% \mathrm{CHO}, 45 \% \\
\text { fat—quantity of hazelnuts not reported) } \\
\text { Change }^{1}\end{array}$} & 5.40 & 3.44 & 1.04 & 2.07 & \\
\hline & & & & & $-0.73^{\mathrm{b}}$ & $-1.22^{\mathrm{b}}$ & +0.08 & -0.40 & \\
\hline \multirow{5}{*}{$\begin{array}{l}\text { Damavandi et al., } \\
2013 \text { [38] }\end{array}$} & \multirow{5}{*}{$\begin{array}{l}\text { Randomised parallel } \\
2 \text { treatments }\end{array}$} & \multirow{5}{*}{$\begin{array}{l}50(16 \mathrm{M}, 34 \mathrm{~F}) \text { with } \\
\text { type } 2 \text { diabetes }\end{array}$} & \multirow{5}{*}{8 weeks } & Baseline & 4.12 & 2.18 & 1.14 & 1.75 & \multirow{5}{*}{$\begin{array}{l}\text { Significantly greater decrease } \\
\text { in HDL-C in the control group } \\
\text { compared to the hazelnut } \\
\text { group }(p=0.009)\end{array}$} \\
\hline & & & & (i) Hazelnuts $10 \%$ of $\mathrm{TE}$ & 3.75 & 2.21 & 1.08 & 1.45 & \\
\hline & & & & Change $^{1}$ & -0.37 & +0.02 & -0.06 & -0.30 & \\
\hline & & & & Baseline & 3.62 & 1.94 & 1.04 & 1.41 & \\
\hline & & & & $\begin{array}{l}\text { (ii) Control (no hazelnuts) } \\
\text { Change }^{1}\end{array}$ & $\begin{array}{c}3.47 \\
-0.15\end{array}$ & $\begin{array}{c}1.90 \\
-0.04\end{array}$ & $\begin{array}{c}0.95 \\
-0.09^{\mathrm{b}}\end{array}$ & $\begin{array}{c}1.40 \\
-0.01\end{array}$ & \\
\hline \multirow{9}{*}{ Deon et al., 2018 [39] } & \multirow{9}{*}{$\begin{array}{l}\text { Randomised parallel } \\
3 \text { treatments }\end{array}$} & \multirow{9}{*}{$\begin{array}{l}66 \text { children and } \\
\text { adolescents ( } 35 \mathrm{M} \\
31 \mathrm{~F}) \text { with } \\
\text { hyperlipidaemia }\end{array}$} & \multirow{9}{*}{8 weeks } & Baseline & 5.58 & 3.67 & 1.60 & $0.76^{\ddagger}$ & \multirow{9}{*}{$\begin{array}{c}\text { No significant between-group } \\
\text { differences }\end{array}$} \\
\hline & & & & $\begin{array}{l}\text { (i) Hazelnuts with skin }(0.43 \mathrm{~g} / \mathrm{kg} \\
(15-30 \mathrm{~g}))\end{array}$ & 5.28 & 3.43 & 1.63 & $0.66 \ddagger$ & \\
\hline & & & & Change ${ }^{1}$ & -0.30 & $-0.24^{\mathrm{a}}$ & +0.03 & -0.10 & \\
\hline & & & & Baseline & 5.73 & 3.66 & 1.58 & $0.69 \ddagger$ & \\
\hline & & & & $\begin{array}{c}\text { (ii) Hazelnuts without skin }(0.43 \mathrm{~g} / \mathrm{kg} \\
(15-30 \mathrm{~g}))\end{array}$ & 5.49 & 3.43 & 1.61 & $0.79 \ddagger$ & \\
\hline & & & & $\begin{array}{l}(15-30 \text { g) }) \\
\text { Change }^{1}\end{array}$ & -0.24 & $-0.23^{a}$ & +0.03 & +0.10 & \\
\hline & & & & Baseline & 5.44 & 3.54 & 1.43 & $0.86 \ddagger$ & \\
\hline & & & & Control (dietary advice only) & 5.28 & 3.41 & 1.44 & $0.87 \ddagger$ & \\
\hline & & & & Change $^{1}$ & -0.16 & -0.13 & +0.01 & +0.01 & \\
\hline
\end{tabular}


Table 4. Cont.

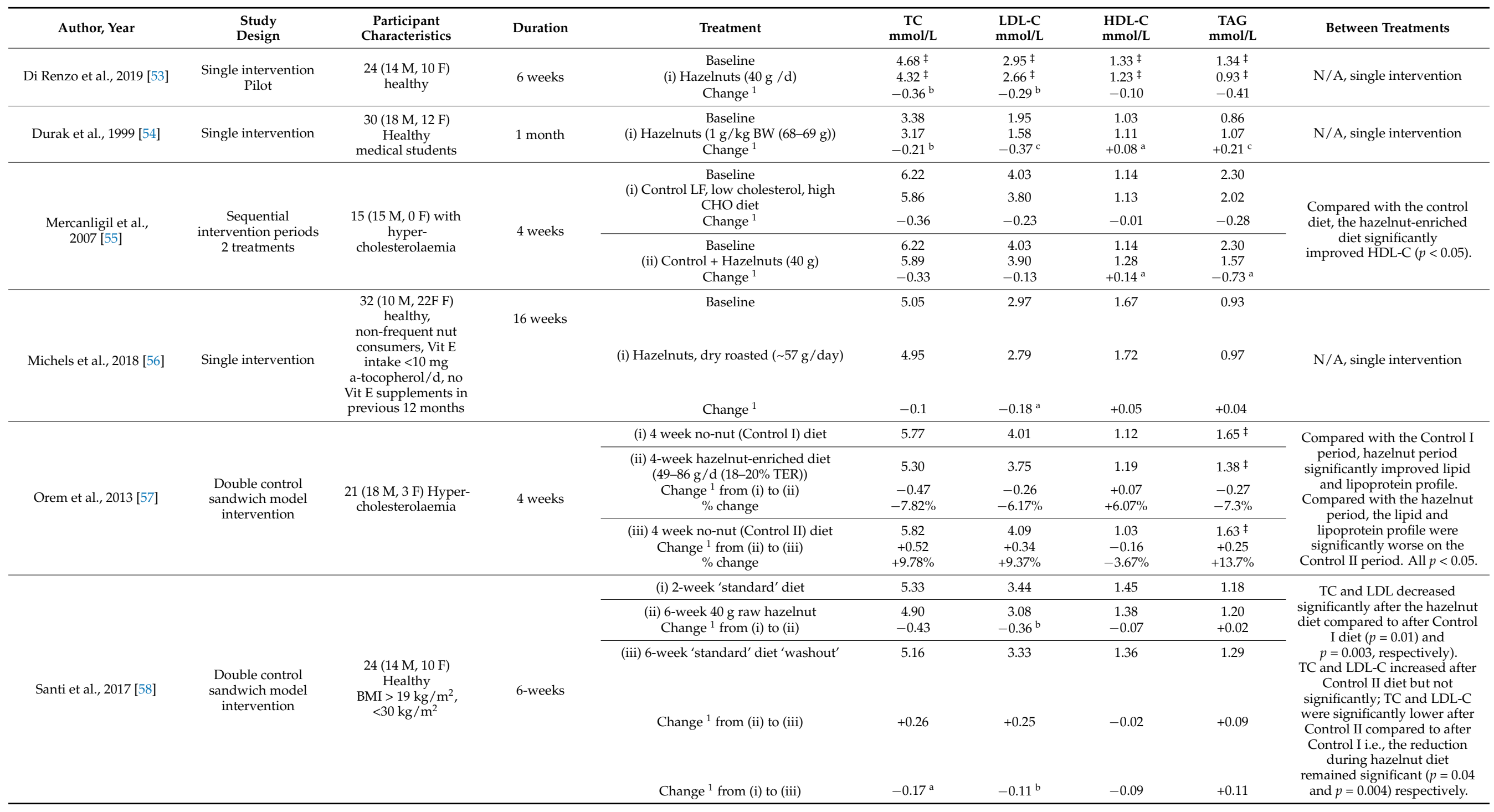


Table 4. Cont.

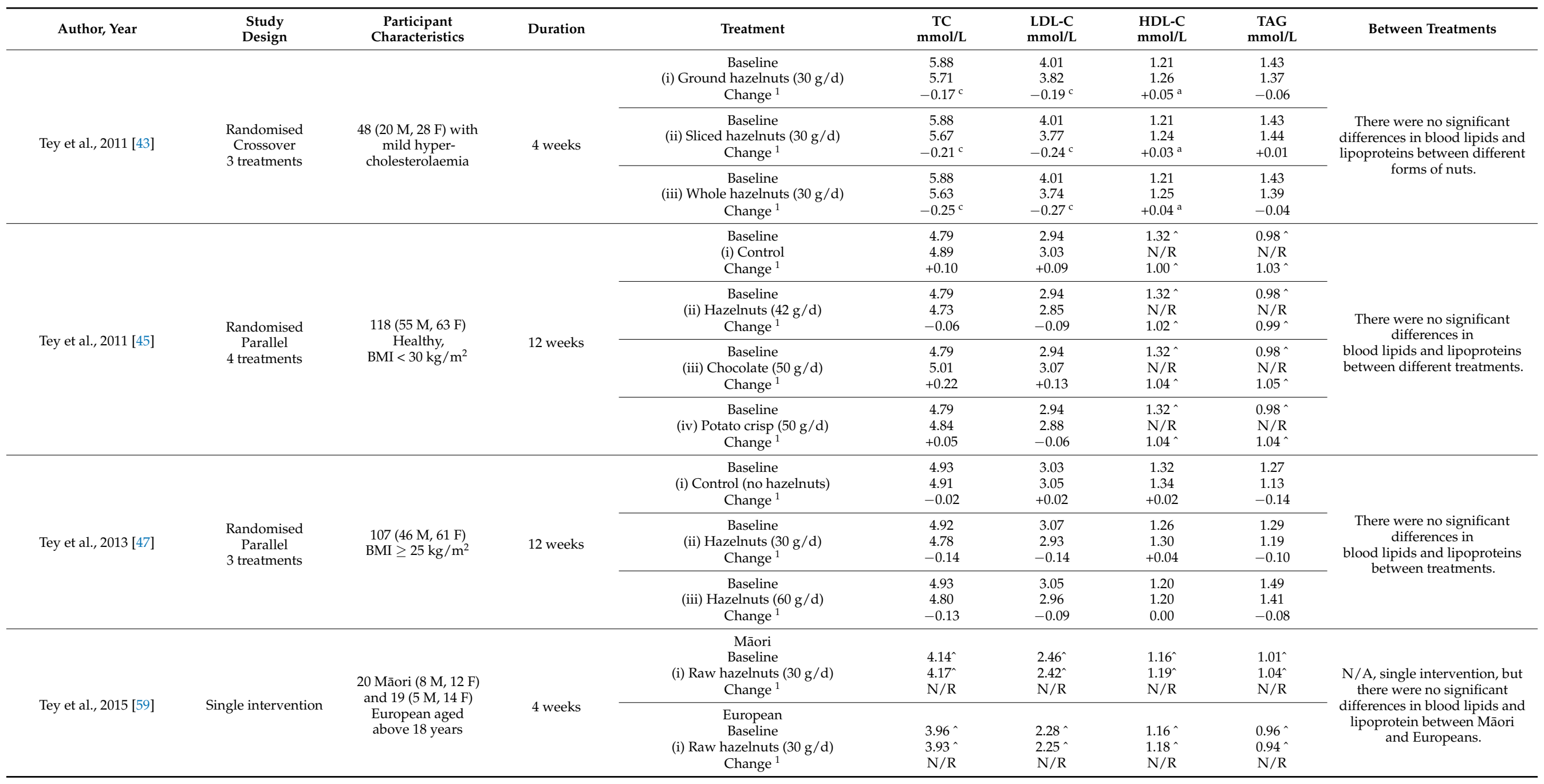


Table 4. Cont.

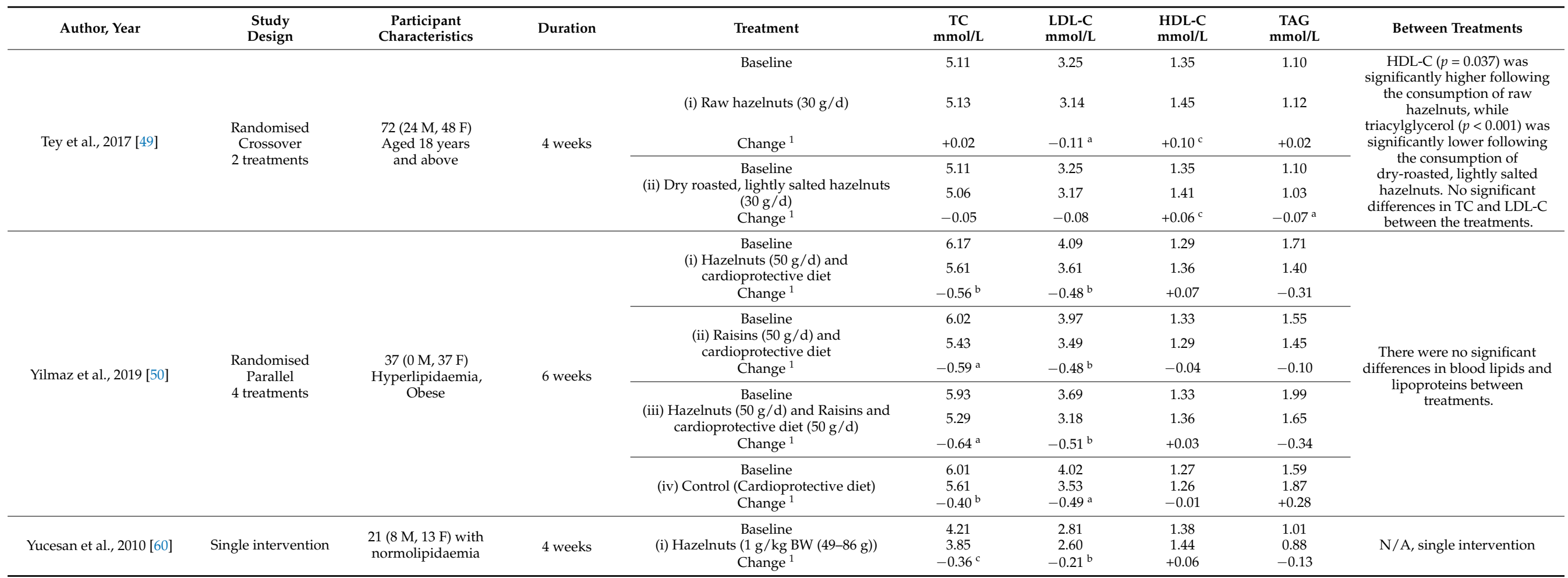

To convert mmol/L TC, LDL-C, HDL-C to $\mathrm{mg} / \mathrm{dL}$ multiply mmol/L by 38.67. To convert mmol/L TAG to mg/dL, multiply mmol/L by 88.57 . Abbreviations used: BW, body weight,

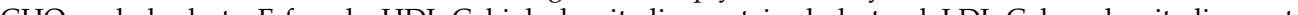
TAG, minus Pre-treatment value (i.e., baseline); ${ }^{a} p<0.05 ;{ }^{b} p<0.01{ }^{c}{ }^{c} p<0.001$; only for those which reported within-group change. ${ }^{\wedge}$ Geometric mean, and differences are ratios of the geometric means; $\ddagger$ Median. 
Table 5. Effects of hazelnut consumption on apolipoprotein A1 and B100 $(n=8)$.

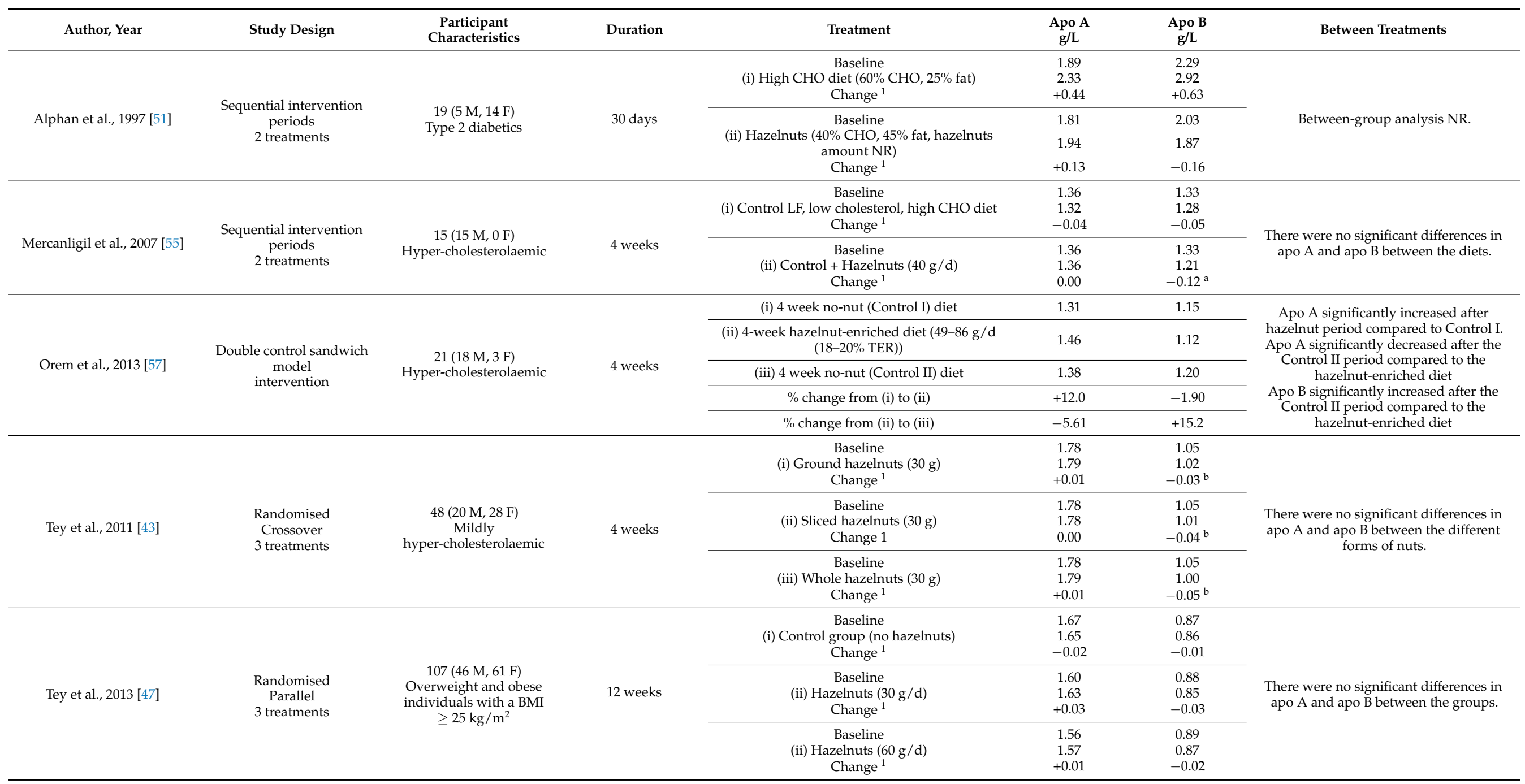


Table 5. Cont.

\begin{tabular}{|c|c|c|c|c|c|c|c|}
\hline Author, Year & Study Design & $\begin{array}{c}\text { Participant } \\
\text { Characteristics }\end{array}$ & Duration & Treatment & $\underset{\mathrm{g} / \mathrm{L}}{\mathrm{Apo} A}$ & $\underset{\mathrm{g} / \mathrm{L}}{\mathrm{Apo} B}$ & Between Treatments \\
\hline \multirow[b]{2}{*}{ Tey et al., 2015 [59] } & \multirow[b]{2}{*}{ Single intervention } & \multirow{2}{*}{$\begin{array}{l}20 \text { Māori }(8 \mathrm{M}, 12 \mathrm{~F}) \text { and } \\
19(5 \mathrm{M}, 14 \mathrm{~F}) \text { European } \\
\text { aged above } 18 \text { years }\end{array}$} & \multirow[b]{2}{*}{4 weeks } & $\begin{array}{c}\text { Māori } \\
\text { Baseline } \\
\text { (i) Raw hazelnuts (30 g/d) } \\
\text { Change }^{1}\end{array}$ & $\begin{array}{l}1.51^{\wedge} \\
1.57^{\wedge} \\
\mathrm{N} / \mathrm{R}\end{array}$ & $\begin{array}{l}0.71^{\wedge} \\
0.70^{\wedge} \\
\mathrm{N} / \mathrm{R}\end{array}$ & \multirow{2}{*}{$\begin{array}{l}\text { N/A, single intervention, but there were } \\
\text { no significant differences in apo A and } \\
\text { apo B between Māori and Europeans. }\end{array}$} \\
\hline & & & & $\begin{array}{c}\text { European } \\
\text { Baseline } \\
\text { (i) Raw hazelnuts }(30 \mathrm{~g} / \mathrm{d}) \\
\text { Change }^{1}\end{array}$ & $\begin{array}{l}1.51^{\wedge} \\
1.52^{\wedge} \\
\mathrm{N} / \mathrm{R}\end{array}$ & $\begin{array}{l}0.65^{\wedge} \\
0.63^{\wedge} \\
\mathrm{N} / \mathrm{R}\end{array}$ & \\
\hline \multirow{4}{*}{ Tey et al., 2017 [49] } & \multirow{4}{*}{$\begin{array}{l}\text { Randomised } \\
\text { Crossover } \\
2 \text { treatments }\end{array}$} & \multirow{4}{*}{$\begin{array}{c}72(24 \mathrm{M}, 48 \mathrm{~F}) \\
\text { Aged } 18 \text { years and above }\end{array}$} & \multirow{4}{*}{4 weeks } & Baseline & 1.59 & 0.87 & \multirow{4}{*}{$\begin{array}{l}\text { There were no significant differences in } \\
\text { apo A and apo B between the groups. }\end{array}$} \\
\hline & & & & \multirow{3}{*}{$\begin{array}{c}\text { Baseline } \\
\text { (ii) Dry roasted, lightly salted hazelnuts (30 } \\
\text { g/d) } \\
\text { Change }^{1}\end{array}$} & 1.59 & 0.87 & \\
\hline & & & & & 1.63 & 0.86 & \\
\hline & & & & & $+0.04^{\mathrm{b}}$ & -0.01 & \\
\hline Yucesan et al., 2010 [60] & Single intervention & $\begin{array}{c}21(8 \mathrm{M}, 13 \mathrm{~F}) \\
\text { Normolipidaemic }\end{array}$ & 4 weeks & $\begin{array}{c}\text { Baseline } \\
\text { (i) Hazelnuts }(1 \mathrm{~g} / \mathrm{kg} \text { BW }(49-86 \mathrm{~g})) \\
\text { Change }^{1}\end{array}$ & $\begin{array}{r}1.35 \\
1.41 \\
+0.06^{\mathrm{b}}\end{array}$ & $\begin{array}{c}0.78 \\
0.71 \\
-0.07^{\mathrm{b}}\end{array}$ & $\mathrm{N} / \mathrm{A}$, single intervention \\
\hline
\end{tabular}

Abbreviations used: apo, apolipoprotein; $\mathrm{BW}$ body weight; $\mathrm{CHO}$ carbohydrate; $\mathrm{F}$, female; LF low fat; $\mathrm{M}$, male; $\mathrm{N} / \mathrm{A}$, not applicable; NR not reported; TE, total energy: $\mathrm{TER}$, tota Abbriation energy requirement. All values are arithmetic means unless otherwise stated. ${ }^{1}$ Change (within-group) $=$ Post-treatment value minus Pre-treatment value (i.e., baseline); ${ }^{\mathrm{b}} p<0.01$; only for those which reported within-group change. ${ }^{\wedge}$ Geometric mean. 
Two studies compared hazelnut consumption to a no-nut control [47,57]. Tey et al. compared the consumption of two doses of hazelnuts ( $30 \mathrm{~g}$ and $60 \mathrm{~g}$ ) to a no-nut control in a parallel study [47]. There were no between-group differences. Orem et al. [57] used a double control sandwich model intervention design. Apo A significantly increased after the hazelnut period compared to control I and decreased again after control period II compared to the hazelnut period. Apo B significantly increased after control period II compared to the hazelnut period.

Two studies included a hazelnut and high carbohydrate group using a sequential design $[51,55]$. Alphan et al. did not report between-group differences, and there were no statistically significant within-group changes [51]. Mercanligil et al. reported no significant differences between the diet groups [55].

Of the single intervention studies, Yucesan et al. reported a significant increase in apo A and a significant decrease in apo B [60], while Tey et al. reported no significant changes [59].

Two studies compared different forms of hazelnuts. Tey et al. showed no significant differences in apo A or B between ground, sliced, and whole nuts, but all three forms were associated with significant reductions in apo B [43]. In a further study, they reported no significant differences for raw versus roasted hazelnuts, but both forms significantly increased apo A compared to baseline [49].

Overall, three of the eight studies reported a significant increase in apo A, and four reported a significant reduction in apo B with hazelnut consumption.

\subsection{Body Weight and Composition}

In total, 17 studies examined the effects of hazelnut consumption on body weight and composition (Table 6). Study designs included: randomised parallel $(n=5)$, randomised crossover $(n=2)$, sequential $(n=3)$, double control sandwich $(n=2)$, and single intervention $(n=5)$. Interventions ranged in duration from 4 to 16 weeks. Sample sizes ranged from 15 to 118 and were heterogeneous in nature. For example, nine comprised healthy participants, five included those with hyperlipidaemia (including one with children), two included those with type 2 diabetes and one specifically recruited people with overweight or obesity.

Ten studies compared hazelnut consumption to a no-nut control. Of these, one study, a sequential intervention study, reported a significant increase in hip circumference and lean body mass, and a significant reduction in fat mass, after the hazelnut diet compared to the standard diet [52]. Nine studies found no significant differences between-groups for any outcome, and one failed to report between-group differences [51]. Of the single intervention studies, one reported a reduction in abdominal circumference [53], and one reported an increase in BMI from baseline [56]. Three studies compared different forms of hazelnuts. One study compared hazelnuts with and without skin [39], one study compared ground vs. sliced vs. whole hazelnuts [43], and a third study compared raw vs. roasted hazelnuts [49]. None of these studies reported any change in body composition. 
Table 6. Effects of hazelnut consumption on body weight $(n=17)$.

\begin{tabular}{|c|c|c|c|c|c|c|c|}
\hline Author, Year & Study Design & $\begin{array}{c}\text { Participant } \\
\text { Characteristics }\end{array}$ & Duration & $\begin{array}{c}\text { Comparison Made } \\
\text { Body Weight }\end{array}$ & $\begin{array}{c}\text { Treatment } \\
\text { Body Weight }\end{array}$ & Change in Body Weight ${ }^{1}$ & Between Treatments \\
\hline \multirow[b]{2}{*}{ Alphan et al., 1997 [51] } & \multirow[b]{2}{*}{$\begin{array}{l}\text { Sequential intervention } \\
\text { periods } \\
2 \text { treatments }\end{array}$} & \multirow[b]{2}{*}{$\begin{array}{c}19(5 \mathrm{M}, 14 \mathrm{~F}) \\
\text { Type } 2 \text { diabetics }\end{array}$} & \multirow[b]{2}{*}{30 days } & $\begin{array}{l}\text { (i) Baseline } \\
\text { BMI: } 27.5 \mathrm{~kg} / \mathrm{m}^{2}\end{array}$ & $\begin{array}{l}\text { (i) High CHO diet (60\% CHO, } 25 \% \text { fat) } \\
\text { BMI: } 27.3 \mathrm{~kg} / \mathrm{m}^{2}\end{array}$ & (i) No significant change & \multirow[b]{2}{*}{$\begin{array}{l}\text { Between-group } \\
\text { analysis NR. }\end{array}$} \\
\hline & & & & $\begin{array}{l}\text { (ii) Baseline } \\
\text { BMI: } 27.1 \mathrm{~kg} / \mathrm{m}^{2}\end{array}$ & $\begin{array}{c}\text { (ii) Hazelnut diet }(40 \% \mathrm{CHO}, 40 \% \text { fat, } \\
\text { Hazelnut amount NR) } \\
\text { BMI: } 27.1 \mathrm{~kg} / \mathrm{m}^{2}\end{array}$ & (ii) No significant change & \\
\hline \multirow{2}{*}{ Damavandi et al., 2012 [37] } & \multirow{2}{*}{$\begin{array}{l}\text { Randomised parallel } \\
2 \text { treatments }\end{array}$} & \multirow{2}{*}{$\begin{array}{c}50(16 \mathrm{M}, 34 \mathrm{~F}) \text { with type } \\
2 \text { diabetes }\end{array}$} & \multirow[b]{2}{*}{8 weeks } & $\begin{array}{c}\text { (i)Baseline } \\
\text { Weight: } 72.13 \mathrm{~kg} \\
\text { BMI: } 28.47 \mathrm{~kg} / \mathrm{m}^{2}\end{array}$ & $\begin{array}{l}\text { (i) Hazelnut }(10 \% \mathrm{TE}) \\
\text { Weight: } 71.47 \mathrm{~kg} \\
\text { BMI: } 27.92 \mathrm{~kg} / \mathrm{m}^{2}\end{array}$ & (i) No significant change & \multirow{2}{*}{$\begin{array}{l}\text { No significant } \\
\text { between-group differences } \\
\text { in body weight or BMI. }\end{array}$} \\
\hline & & & & $\begin{array}{c}\text { (ii) Baseline } \\
\text { Weight: } 71.98 \mathrm{~kg} \\
\text { BMI: } 28.18 \mathrm{~kg} / \mathrm{m}^{2}\end{array}$ & $\begin{array}{l}\text { (ii) Control: no hazelnuts } \\
\text { Weight: } 71.64 \mathrm{~kg} \\
\text { BMI: } 28.08 \mathrm{~kg} / \mathrm{m}^{2}\end{array}$ & (ii) No significant change & \\
\hline \multirow{3}{*}{ Deon et al., 2018 [39] } & \multirow{3}{*}{$\begin{array}{l}\text { Randomised parallel } \\
3 \text { treatments }\end{array}$} & \multirow{3}{*}{$\begin{array}{l}66 \text { children and } \\
\text { adolescents ( } 35 \text { M } 31 \text { F) } \\
\text { with hyperlipidaemia }\end{array}$} & \multirow{3}{*}{8 weeks } & $\begin{array}{l}\text { (i) Baseline } \\
\text { Weight: } 44.4 \mathrm{~kg} \\
\text { BMI: } 20.4 \mathrm{~kg} / \mathrm{m}^{2}\end{array}$ & $\begin{array}{c}\text { (i) Hazelnuts with skin }(0.43 \mathrm{~g} / \mathrm{kg}(15-30 \\
\text { g/d })) \\
\text { Weight: } 45.0 \mathrm{~kg} \\
\text { BMI: } 20.3 \mathrm{~kg} / \mathrm{m}^{2}\end{array}$ & $\begin{array}{l}\text { (i) No significant change } \\
\text { for BMI }\end{array}$ & \multirow{3}{*}{$\begin{array}{l}\text { No significant } \\
\text { between-group differences } \\
\text { in BMI, there was a time } \\
\text { effect for height } \\
\text { and weight. }\end{array}$} \\
\hline & & & & $\begin{array}{l}\text { (ii) Baseline } \\
\text { Weight: } 47.8 \mathrm{~kg} \\
\text { BMI: } 20.3 \mathrm{~kg} / \mathrm{m}^{2}\end{array}$ & $\begin{array}{l}\text { (ii) Hazelnuts without skin }(0.43 \mathrm{~g} / \mathrm{kg} \\
\text { (15-30 g/d)) } \\
\text { Weight: } 48.4 \mathrm{~kg} \\
\text { BMI: } 20.3 \mathrm{~kg} / \mathrm{m}^{2}\end{array}$ & $\begin{array}{l}\text { (ii) No significant change } \\
\text { for BMI }\end{array}$ & \\
\hline & & & & $\begin{array}{l}\text { (iii) Baseline } \\
\text { Weight: } 49.5 \mathrm{~kg} \\
\text { BMI: } 20.9 \mathrm{~kg} / \mathrm{m}^{2}\end{array}$ & $\begin{array}{l}\text { (iii) Control: no hazelnuts } \\
\text { Weight: } 50.0 \mathrm{~kg} \\
\text { BMI: } 20.8 \mathrm{~kg} / \mathrm{m}^{2}\end{array}$ & $\begin{array}{l}\text { (iii) No significant change } \\
\text { for BMI }\end{array}$ & \\
\hline \multirow[t]{2}{*}{ Di Renzo et al., 2014 [52] } & \multirow{2}{*}{$\begin{array}{l}\text { Sequential intervention } \\
\text { periods } \\
2 \text { treatments }\end{array}$} & \multirow{2}{*}{$\begin{array}{l}24 \text { participants } \\
\text { BMI } \geq 19 \mathrm{~kg} / \mathrm{m}^{2}\end{array}$} & \multirow[t]{2}{*}{4 weeks } & $\begin{array}{c}\text { (i) Baseline } \\
\text { Weight: } 66.15 \mathrm{~kg}\end{array}$ & $\begin{array}{c}\text { (i) } 4 \text { week standard diet (Italian } \\
\text { Mediterranean diet) } \\
\text { Weight: } 67.8 \mathrm{~kg} \\
\text { WC: } 77.44 \mathrm{~cm} \\
\text { HC: } 97.5 \mathrm{~cm} \\
\text { Fat mass: } 16.93 \mathrm{~kg} \\
\text { LBM: } 34.56 \mathrm{~kg} \\
\end{array}$ & \multirow[t]{2}{*}{ NR } & \multirow{2}{*}{$\begin{array}{l}\text { HC and LBM was } \\
\text { significantly higher, and fat } \\
\text { mass was significantly } \\
\text { lower after the hazelnut } \\
\text { diet compared to the } \\
\text { standard diet (all } p<0.05 \text { ). }\end{array}$} \\
\hline & & & & $\begin{array}{l}\text { (ii) Baseline } \\
\text { NR }\end{array}$ & $\begin{array}{l}\text { (ii) Hazelnuts }(40 \mathrm{~g} / \mathrm{d}) \\
\text { Weight: } 66.8 \mathrm{~kg} \\
\text { WC: } 76.43 \mathrm{~cm} \\
\text { HC: } 99.76 \mathrm{~cm} \\
\text { Fat mass: } 14.83 \mathrm{~kg} \\
\text { LBM: } 35.07 \mathrm{~kg}\end{array}$ & & \\
\hline
\end{tabular}


Table 6. Cont.

\begin{tabular}{|c|c|c|c|c|c|c|c|}
\hline Author, Year & Study Design & $\begin{array}{c}\text { Participant } \\
\text { Characteristics }\end{array}$ & Duration & $\begin{array}{c}\text { Comparison Made } \\
\text { Body Weight } \\
\end{array}$ & $\begin{array}{c}\text { Treatment } \\
\text { Body Weight }\end{array}$ & Change in Body Weight ${ }^{1}$ & Between Treatments \\
\hline Di Renzo et al., 2019 [53] & $\begin{array}{l}\text { Single intervention } \\
\text { Pilot }\end{array}$ & $\begin{array}{l}24(14 \mathrm{M}, 10 \mathrm{~F}) \\
\text { healthy }\end{array}$ & 6 weeks & $\begin{array}{c}\text { (i) Baseline }{ }^{\ddagger} \\
\text { Weight: } 71.4 \mathrm{~kg} \\
\text { BMI: } 25.95 \mathrm{~kg} / \mathrm{m}^{2} \\
\text { WC: } 86.25 \mathrm{~cm} \\
\text { AC: } 94.00 \mathrm{~cm} \\
\text { HC: } 98.25 \mathrm{~cm} \\
\text { Total body fat: } 34.75 \mathrm{~kg} \\
\text { Total BF: } 29.65 \% \\
\text { Android BF: } 28.75 \% \\
\text { Gynoid BF: } 21.08 \% \\
\text { LBM: } 47.63 \mathrm{~kg} \\
\text { ASMMI: } 8.37\end{array}$ & $\begin{array}{l}\text { (i) Hazelnuts }(40 \mathrm{~g} / \mathrm{d} \text { ) } \\
\text { Weight: } 71.05 \mathrm{~kg} \\
\text { BMI: } 25.76 \mathrm{~kg} / \mathrm{m}^{2} \\
\text { WC: } 85.00 \mathrm{~cm} \\
\text { AC: } 93.50 \mathrm{~cm} \\
\text { HC: } 99.00 \mathrm{~cm} \\
\text { Total body fat: } 34.95 \mathrm{~kg} \\
\text { Total BF: } 29.05 \% \\
\text { Android BF: } 28.80 \% \\
\text { Gynoid BF } 21.34 \% \\
\text { LBM: } 48.09 \mathrm{~kg} \\
\text { ASMMI: } 8.05\end{array}$ & $\begin{array}{l}\text { (i) AC was significantly } \\
\text { lower after the hazelnut } \\
\text { intervention }\end{array}$ & N/A, single intervention \\
\hline Durak et al., 1999 [54] & Single intervention & $\begin{array}{l}30(18 \mathrm{M}, 12 \mathrm{~F}) \\
\text { Healthy } \\
\text { Medical students }\end{array}$ & 1 month & $\begin{array}{l}\text { (i) Habitual diet } \\
\text { Weight: } 68.7 \mathrm{~kg}\end{array}$ & $\begin{array}{c}\text { (i) Hazelnuts (1 g/kg BW }(68-69 \mathrm{~g})) \\
\text { Weight: } 69.2 \mathrm{~kg}\end{array}$ & (i) No significant change & N/A, single intervention \\
\hline \multirow{2}{*}{ Mercanligil et al., 2007 [55] } & \multirow{2}{*}{$\begin{array}{l}\text { Sequential intervention } \\
\text { periods } \\
2 \text { treatments }\end{array}$} & \multirow{2}{*}{$\begin{array}{c}15(15 \mathrm{M}, 0 \mathrm{~F}) \\
\text { Hyper-cholesterolaemic }\end{array}$} & \multirow{2}{*}{4 weeks } & $\begin{array}{c}\text { (i) Baseline } \\
\text { Weight: } 74.3 \mathrm{~kg}\end{array}$ & $\begin{array}{c}\text { (i) Control LF, high CHO diet } \\
\text { Weight: } 74.2 \mathrm{~kg}\end{array}$ & (i) No significant change & \multirow{2}{*}{$\begin{array}{c}\text { No significant } \\
\text { between-group differences } \\
\text { in body weight. }\end{array}$} \\
\hline & & & & $\begin{array}{c}\text { (ii) Baseline } \\
\text { Weight: } 74.3 \mathrm{~kg}\end{array}$ & $\begin{array}{l}\text { (ii) Control + Hazelnuts }(40 \mathrm{~g} / \mathrm{d}) \\
\text { Weight: } 74.0 \mathrm{~kg}\end{array}$ & (ii) No significant change & \\
\hline Michels et al., 2018 [56] & Single intervention & $\begin{array}{c}32(10 \mathrm{M}, 22 \mathrm{~F} \mathrm{~F}) \text { healthy, } \\
\text { non-frequent nut } \\
\text { consumers, Vit E intake } \\
<10 \mathrm{mg} \text { a-tocopherol/d, no } \\
\text { Vit E supplements in } \\
\text { previous } 12 \text { months }\end{array}$ & 16 weeks & $\begin{array}{l}\text { (i) Baseline } \\
\text { BMI: } 26.1 \mathrm{~kg} / \mathrm{m}^{2}\end{array}$ & $\begin{array}{l}\text { (i) Hazelnuts, dry roasted ( } \sim 57 \mathrm{~g} / \text { day }) \\
\text { BMI: } 26.3 \mathrm{~kg} / \mathrm{m}^{2}\end{array}$ & $\begin{array}{c}\text { (i) BMI: }+0.2 \mathrm{~kg} / \mathrm{m}^{2} \\
(p=0.009)\end{array}$ & N/A, single intervention \\
\hline \multirow{2}{*}{ Orem et al., 2013 [57] } & \multirow{2}{*}{$\begin{array}{l}\text { Double control sandwich } \\
\text { model } \\
\text { intervention }\end{array}$} & \multirow{2}{*}{$\begin{array}{l}21(18 \mathrm{M}, 3 \mathrm{~F}) \\
\text { Hyper-cholesterolaemic }\end{array}$} & \multirow{2}{*}{4 weeks } & $\begin{array}{l}\text { (i) } 4 \text { week no-nut } \\
\text { (Control I) diet } \\
\text { Weight: } 81.0 \mathrm{~kg} \\
\text { BMI: } 27.4 \mathrm{~kg} / \mathrm{m}^{2}\end{array}$ & $\begin{array}{c}\text { (ii) 4-week hazelnut-enriched diet } \\
(49-86 \mathrm{~g} / \mathrm{d} \\
(18-20 \% \mathrm{TER})) \\
\text { Weight: } 79.1 \mathrm{~kg} \\
\text { BMI: } 26.9 \mathrm{~kg} / \mathrm{m}^{2} \\
\end{array}$ & $\begin{array}{c}\text { (i) to (ii): } \\
\text { Weight: }-0.9 \mathrm{~kg} ;-2.3 \% \\
\text { BMI: }-0.5 \mathrm{~kg} / \mathrm{m}^{2} ;-2.02 \%\end{array}$ & \multirow{2}{*}{$\begin{array}{c}\text { Body weight and BMI } \\
\text { were significantly different } \\
\text { between (i) and (ii) and } \\
\text { between (i) and (iii). There } \\
\text { was no significant } \\
\text { difference in body weight } \\
\text { or BMI between (ii) } \\
\text { and (iii). }\end{array}$} \\
\hline & & & & $\begin{array}{c}\text { (ii) } 4 \text {-week } \\
\text { hazelut-enriched diet } \\
\text { (49-86 g/d } \\
(18-20 \% \text { TER)) } \\
\text { Weight: } 79.1 \mathrm{~kg} \\
\text { BMI: } 26.9 \mathrm{~kg} / \mathrm{m}^{2}\end{array}$ & $\begin{array}{c}\text { (iii) } 4 \text { week no-nut (Control II) diet } \\
\text { Weight: } 79.5 \mathrm{~kg} \\
\text { BMI: } 26.9 \mathrm{~kg} / \mathrm{m}^{2}\end{array}$ & $\begin{array}{c}\text { (ii) to (iii): } \\
\text { Weight: }+0.4 \mathrm{~kg} ;+0.4 \% \\
\text { BMI: no numerical change; } \\
+0.07 \%\end{array}$ & \\
\hline
\end{tabular}


Table 6. Cont.

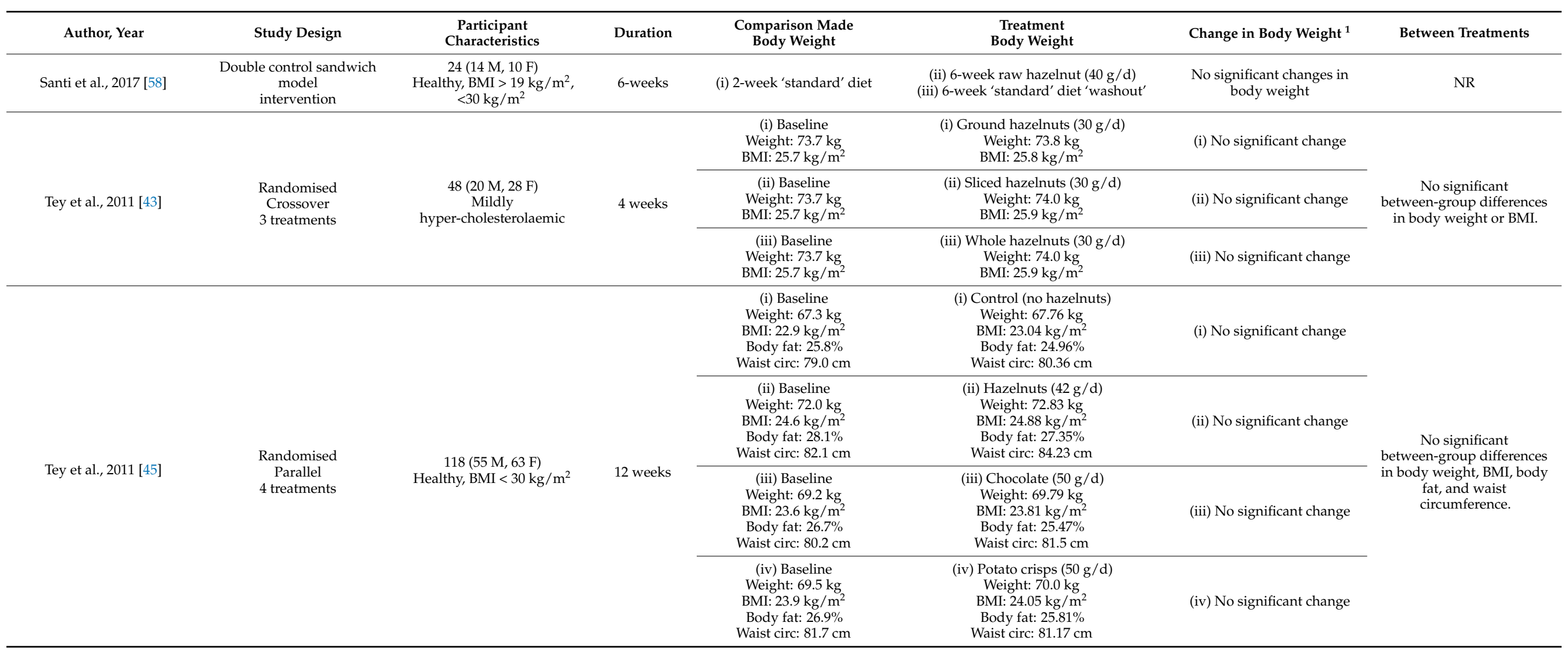


Table 6. Cont.

\begin{tabular}{|c|c|c|c|c|c|c|c|}
\hline Author, Year & Study Design & $\begin{array}{c}\text { Participant } \\
\text { Characteristics }\end{array}$ & Duration & $\begin{array}{c}\text { Comparison Made } \\
\text { Body Weight }\end{array}$ & $\begin{array}{c}\text { Treatment } \\
\text { Body Weight }\end{array}$ & Change in Body Weight ${ }^{1}$ & Between Treatments \\
\hline \multirow{3}{*}{ Tey et al., 2013 [47] } & \multirow{3}{*}{$\begin{array}{l}\text { Randomised } \\
\text { Parallel } \\
3 \text { treatments }\end{array}$} & \multirow{3}{*}{$\begin{array}{c}107(46 \mathrm{M}, 61 \mathrm{~F}) \\
\text { Overweight and obese } \\
\text { individuals with a } \\
\mathrm{BMI} \geq 25 \mathrm{~kg} / \mathrm{m}^{2}\end{array}$} & \multirow{3}{*}{12 weeks } & $\begin{array}{c}\text { (i) Baseline } \\
\text { Weight: } 88.7 \mathrm{~kg} \\
\text { BMI: } 30.4 \mathrm{~kg} / \mathrm{m}^{2} \\
\text { Body fat: } 33.9 \% \\
\text { Fat mass: } 30.1 \mathrm{~kg} \\
\text { Fat-free mass: } 58.7 \mathrm{~kg} \\
\end{array}$ & $\begin{array}{l}\text { (i) Control (no hazelnuts) } \\
\text { Weight: } 88.7 \mathrm{~kg} \\
\text { BMI: } 30.4 \mathrm{~kg} / \mathrm{m}^{2} \\
\text { Body fat: } 33.9 \% \\
\text { Fat mass: } 30.1 \mathrm{~kg} \\
\text { Fat-free mass: } 58.6 \mathrm{~kg} \\
\end{array}$ & (i) No significant change & \multirow{3}{*}{$\begin{array}{l}\text { There were no significant } \\
\text { differences in body weight } \\
\text { BMI, body fat percent, fat } \\
\text { mass, and fat-free mass } \\
\text { between the treatments. }\end{array}$} \\
\hline & & & & $\begin{array}{c}\text { (ii) Baseline } \\
\text { Weight: } 86.2 \mathrm{~kg} \\
\text { BMI: } 30.7 \mathrm{~kg} / \mathrm{m}^{2} \\
\text { Body fat: } 35.4 \% \\
\text { Fat mass: } 30.7 \mathrm{~kg} \\
\text { Fat-free mass: } 55.5 \mathrm{~kg}\end{array}$ & $\begin{array}{l}\text { (ii) Hazelnuts }(30 \mathrm{~g} / \mathrm{d}) \\
\text { Weight: } 86.2 \mathrm{~kg} \\
\text { BMI: } 30.7 \mathrm{~kg} / \mathrm{m}^{2} \\
\text { Body fat: } 35.4 \% \\
\text { Fat mass: } 30.7 \mathrm{~kg} \\
\text { Fat-free mass: } 55.6 \mathrm{~kg}\end{array}$ & (ii) No significant change & \\
\hline & & & & $\begin{array}{c}\text { (iii) Baseline } \\
\text { Weight: } 92.0 \mathrm{~kg} \\
\text { BMI: } 30.9 \mathrm{~kg} / \mathrm{m}^{2} \\
\text { Body fat: } 35.0 \% \\
\text { Fat mass: } 32.5 \mathrm{~kg} \\
\text { Fat-free mass: } 59.5 \mathrm{~kg} \\
\end{array}$ & $\begin{array}{l}\text { (iii) Hazelnuts }(60 \mathrm{~g} / \mathrm{d}) \\
\text { Weight: } 92.2 \mathrm{~kg} \\
\text { BMI: } 30.9 \mathrm{~kg} / \mathrm{m}^{2} \\
\text { Body fat: } 34.9 \% \\
\text { Fat mass: } 32.5 \mathrm{~kg} \\
\text { Fat-free mass: } 59.7 \mathrm{~kg} \\
\end{array}$ & (iii) No significant change & \\
\hline \multirow{2}{*}{ Tey et al., 2015 [59] } & \multirow{2}{*}{ Single intervention } & \multirow{2}{*}{$\begin{array}{c}20 \text { Māori }(8 \mathrm{M}, 12 \mathrm{~F}) \text { and } 19 \\
(5 \mathrm{M}, 14 \mathrm{~F}) \text { European aged } \\
\text { above } 18 \text { years }\end{array}$} & \multirow{2}{*}{4 weeks } & $\begin{array}{c}\text { Māori } \\
\text { (i) Baseline } \\
\text { Weight }{ }^{\wedge}: 76.3 \mathrm{~kg} \\
\text { BMI }^{\wedge}: 25.5 \mathrm{~kg} / \mathrm{m}^{2} \\
\text { Body fat }^{\wedge}: 26.9 \%\end{array}$ & $\begin{array}{c}\text { Māori } \\
\text { (i) Hazelnuts }(30 \mathrm{~g} / \mathrm{d}) \\
\text { Weight }^{\wedge}: 76.3 \mathrm{~kg} \\
\text { BMI }^{\wedge}: 25.5 \mathrm{~kg} / \mathrm{m}^{2} \\
\text { Body fat }^{\wedge}: 27.3 \%\end{array}$ & $\begin{array}{l}\text { Māori } \\
\text { (i) No significant change }\end{array}$ & \multirow{2}{*}{$\begin{array}{l}\text { N/A, single intervention, } \\
\text { but there were no } \\
\text { significant differences in } \\
\text { body weight, BMI, and } \\
\text { body fat percent between } \\
\text { Māori and Europeans. }\end{array}$} \\
\hline & & & & $\begin{array}{c}\text { European } \\
\text { (ii) Baseline } \\
\text { Weight } \wedge: 71.5 \mathrm{~kg} \\
\text { BMI }^{\wedge}: 24.4 \mathrm{~kg} / \mathrm{m}^{2} \\
\text { Body fat }{ }^{\wedge}: 25.9 \%\end{array}$ & $\begin{array}{c}\text { European } \\
\text { (ii) Hazelnuts }(30 \mathrm{~g} / \mathrm{d}) \\
\text { Weight }{ }^{\wedge}: 71.8 \mathrm{~kg} \\
\text { BMI }:: 24.4 \mathrm{~kg} / \mathrm{m}^{2} \\
\text { Body fat }{ }^{\wedge}: 26.6 \%\end{array}$ & $\begin{array}{c}\text { European } \\
\text { (i) No significant change }\end{array}$ & \\
\hline
\end{tabular}


Table 6. Cont.

\begin{tabular}{|c|c|c|c|c|c|c|c|}
\hline Author, Year & Study Design & $\begin{array}{c}\text { Participant } \\
\text { Characteristics }\end{array}$ & Duration & $\begin{array}{l}\text { Comparison Made } \\
\text { Body Weight }\end{array}$ & $\begin{array}{c}\text { Treatment } \\
\text { Body Weight }\end{array}$ & Change in Body Weight ${ }^{1}$ & Between Treatments \\
\hline \multirow[b]{2}{*}{ Tey et al., 2017 [49] } & \multirow{2}{*}{$\begin{array}{l}\text { Randomised } \\
\text { Crossover } \\
2 \text { treatments }\end{array}$} & \multirow{2}{*}{$\begin{array}{c}72(24 \mathrm{M}, 48 \mathrm{~F}) \\
\text { Aged } 18 \text { years and above }\end{array}$} & \multirow[b]{2}{*}{4 weeks } & $\begin{array}{c}\text { (i) Baseline } \\
\text { Weight: } 76.7 \mathrm{~kg} \\
\text { BMI: } 26.7 \mathrm{~kg} / \mathrm{m}^{2} \\
\text { Body fat: } 32.0 \% \\
\text { Fat mass: } 25.1 \mathrm{~kg} \\
\text { Fat-free mass: } 51.6 \mathrm{~kg}\end{array}$ & $\begin{array}{l}\text { (i) Raw hazelnuts }(30 \mathrm{~g} / \mathrm{d}) \\
\text { Weight: } 76.57 \mathrm{~kg} \\
\text { BMI: } 26.65 \mathrm{~kg} / \mathrm{m}^{2} \\
\text { Body fat: } 31.83 \% \\
\text { Fat mass: } 24.83 \mathrm{~kg} \\
\text { Fat-free mass: } 51.71 \mathrm{~kg}\end{array}$ & (i) No significant change & \multirow{2}{*}{$\begin{array}{l}\text { There were no significant } \\
\text { differences in body weight, } \\
\text { BMI, body fat, fat mass, } \\
\text { and fat-free mass between } \\
\text { the treatments. }\end{array}$} \\
\hline & & & & $\begin{array}{c}\text { (ii) Baseline } \\
\text { Weight: } 76.7 \mathrm{~kg} \\
\text { BMI: } 26.7 \mathrm{~kg} / \mathrm{m}^{2} \\
\text { Body fat: } 32.0 \% \\
\text { Fat mass: } 25.1 \mathrm{~kg} \\
\text { Fat-free mass: } 51.6 \mathrm{~kg}\end{array}$ & $\begin{array}{c}\text { (ii) Dry roasted, lightly salted hazelnuts } \\
\text { (30 g/d) } \\
\text { Weight: } 76.67 \mathrm{~kg} \\
\text { BMI: } 26.68 \mathrm{~kg} / \mathrm{m}^{2} \\
\text { Body fat: } 31.86 \% \\
\text { Fat mass: } 24.96 \mathrm{~kg} \\
\text { Fat-free mass: } 51.69 \mathrm{~kg}\end{array}$ & (ii) No significant change & \\
\hline \multirow{2}{*}{ Yilmaz et al., 2019 [50] } & \multirow{2}{*}{$\begin{array}{l}\text { Randomised } \\
\text { Parallel } \\
4 \text { treatments }\end{array}$} & \multirow{2}{*}{$\begin{array}{c}37(0 \mathrm{M}, 37 \mathrm{~F}) \\
\text { Hyperlipidaemia, Obese }\end{array}$} & \multirow{2}{*}{6 weeks } & $\begin{array}{c}\text { (i) Baseline } \\
\text { Weight: } 78.5 \mathrm{~kg} \\
\text { BMI: } 35.7 \mathrm{~kg} / \mathrm{m}^{2} \\
\text { Waist circ: } 104.1 \mathrm{~cm} \\
\text { Waist } / \text { Hip ratio: } 0.88 \\
\text { Fat mass: } 34.0 \mathrm{~kg} \\
\text { Fat mass: } 43.1 \%\end{array}$ & $\begin{array}{l}\text { (i) Hazelnuts }(50 \mathrm{~g} / \mathrm{d} \text { ) and } \\
\text { cardioprotective diet } \\
\text { Weight: } 76.0 \mathrm{~kg} \\
\text { BMI: } 34.5 \mathrm{~kg} / \mathrm{m}^{2} \\
\text { Waist circ: } 98.3 \mathrm{~cm} \\
\text { Waist/Hip ratio: } 0.85 \\
\text { Fat mass: } 31.8 \mathrm{~kg} \\
\text { Fat mass: } 41.7 \%\end{array}$ & $\begin{array}{c}\text { (i) Hazelnuts }(50 \mathrm{~g} / \mathrm{d}) \\
\text { Weight: }-2.5 \mathrm{~kg}(p=0.030) \\
\text { BMI: }-1.2 \mathrm{~kg} / \mathrm{m}^{2} \\
(p=0.031) \\
\text { Waist circ: }-5.7 \mathrm{~cm} \\
(p=0.113) \\
\text { Waist } / \text { Hip ratio: }-0.03 \\
(p=0.650) \\
\text { Fat mass: }-2.21 \mathrm{~kg} \\
(p=0.005) \\
\text { Fat mass: }-1.41 \% \\
(p=0.001)\end{array}$ & \multirow{2}{*}{$\begin{array}{l}\text { There were no significant } \\
\text { differences in body weight } \\
\text { BMI, waist circumference, } \\
\text { waist/hip ratio, and fat } \\
\text { mass between } \\
\text { the treatments. }\end{array}$} \\
\hline & & & & $\begin{array}{c}\text { (ii) Baseline } \\
\text { Weight: } 83.7 \mathrm{~kg} \\
\text { BMI: } 35.8 \mathrm{~kg} / \mathrm{m}^{2} \\
\text { Waist circ: } 106.1 \mathrm{~cm} \\
\text { Waist } / \text { Hip ratio: } 0.89 \\
\text { Fat mass: } 35.4 \mathrm{~kg} \\
\text { Fat mass: } 41.9 \%\end{array}$ & $\begin{array}{l}\text { (ii) Raisins }(50 \mathrm{~g} / \mathrm{d}) \text { and } \\
\text { cardioprotective diet } \\
\text { Weight: } 82.2 \mathrm{~kg} \\
\text { BMI: } 35.1 \mathrm{~kg} / \mathrm{m}^{2} \\
\text { Waist circ: } 101.2 \mathrm{~cm} \\
\text { Waist } / \text { Hip ratio: } 0.86 \\
\text { Fat mass: } 34.1 \mathrm{~kg} \\
\text { Fat mass: } 41.1 \%\end{array}$ & $\begin{array}{c}\text { (ii) Raisins }(50 \mathrm{~g} / \mathrm{d}) \\
\text { Weight: }-1.5 \mathrm{~kg}(p=0.074) \\
\text { BMI: }-0.7 \mathrm{~kg} / \mathrm{m}^{2} \\
(p=0.046) \\
\text { Waist circ: }-4.9 \mathrm{~cm} \\
(p=0.0001) \\
\text { Waist } / \text { Hip ratio: }-0.03 \\
(p=0.009) \\
\text { Fat mass: }-1.32 \mathrm{~kg} \\
(p=0.021) \\
\text { Fat mass: }-0.90 \% \\
(p=0.241)\end{array}$ & \\
\hline
\end{tabular}


Table 6. Cont.

\begin{tabular}{|c|c|c|c|c|c|c|c|}
\hline Author, Year & Study Design & $\begin{array}{c}\text { Participant } \\
\text { Characteristics } \\
\end{array}$ & Duration & $\begin{array}{c}\text { Comparison Made } \\
\text { Body Weight } \\
\end{array}$ & $\begin{array}{c}\text { Treatment } \\
\text { Body Weight }\end{array}$ & Change in Body Weight ${ }^{1}$ & Between Treatments \\
\hline & & & & $\begin{array}{c}\text { (iii) Baseline } \\
\\
\text { Weight: } 80.0 \mathrm{~kg} \\
\text { BMI: } 34.6 \mathrm{~kg} / \mathrm{m}^{2} \\
\text { Waist circ: } 98.3 \mathrm{~cm} \\
\text { Waist/Hip ratio: } 0.85 \\
\text { Fat mass: } 33.3 \mathrm{~kg} \\
\text { Fat mass: } 41.4 \%\end{array}$ & $\begin{array}{l}\text { (iii) Hazelnuts }(50 \mathrm{~g} / \mathrm{d} \text { ) and Raisins } \\
(50 \mathrm{~g} / \mathrm{d} \text { ) and cardioprotective diet } \\
\text { Weight: } 77.9 \mathrm{~kg} \\
\text { BMI: } 33.6 \mathrm{~kg} / \mathrm{m}^{2} \\
\text { Waist circ: } 95.1 \mathrm{~cm} \\
\text { Waist } / \text { Hip ratio: } 0.85 \\
\text { Fat mass: } 31.1 \mathrm{~kg} \\
\text { Fat mass: } 39.6 \%\end{array}$ & $\begin{array}{c}\text { (iii) Hazelnuts }(50 \mathrm{~g} / \mathrm{d}) \\
\text { and Raisins }(50 \mathrm{~g} / \mathrm{d}) \\
\text { Weight: }-2.1 \mathrm{~kg}(p=0.002) \\
\text { BMI: }-0.9 \mathrm{~kg} / \mathrm{m}^{2} \\
(p=0.004) \\
\text { Waist circ: }-3.2 \mathrm{~cm} \\
(p=0.122) \\
\text { Waist } / \text { Hip ratio: }-0.01 \\
(p=1.000) \\
\text { Fat mass: }-2.26 \mathrm{~kg} \\
(p=0.001) \\
\text { Fat mass: }-1.72 \% \\
(p=0.002)\end{array}$ & \\
\hline & & & & $\begin{array}{l}\text { (iv) Baseline } \\
\text { Weight: } 81.9 \mathrm{~kg} \\
\text { BMI: } 36.0 \mathrm{~kg} / \mathrm{m}^{2} \\
\text { Waist circ: } 108.1 \mathrm{~cm} \\
\text { Waist } / \text { Hip ratio: } 0.91 \\
\text { Fat mass: } 35.7 \mathrm{~kg} \\
\text { Fat mass: } 43.4 \%\end{array}$ & $\begin{array}{c}\text { (iv) Control (Cardioprotective diet) } \\
\text { Weight: } 79.6 \mathrm{~kg} \\
\text { BMI: } 34.9 \mathrm{~kg} / \mathrm{m}^{2} \\
\text { Waist circ: } 99.9 \mathrm{~cm} \\
\text { Waist } / \text { Hip ratio: } 0.87 \\
\text { Fat mass: } 33.5 \mathrm{~kg} \\
\text { Fat mass: } 41.9 \%\end{array}$ & $\begin{array}{c}\text { (iv) Control } \\
\text { (Cardioprotective diet) } \\
\text { Weight: }-2.4 \mathrm{~kg}(p=0.017) \\
\text { BMI: }-1.1 \mathrm{~kg} / \mathrm{m}^{2} \\
(p=0.020) \\
\text { Waist circ: }-8.2 \mathrm{~cm} \\
(p=0.002) \\
\text { Waist } / \text { Hip ratio: } \\
(p=0.009) \\
\text { Fat mass: }-2.17 \mathrm{~kg} \\
(p=0.002) \\
\text { Fat mass: }-1.42 \% \\
(p=0.003)\end{array}$ & \\
\hline
\end{tabular}




\subsection{Blood Pressure}

In total, seven studies examined the effects of hazelnut consumption on blood pressure (Table 7) $[39,47,49,50,53,56,59]$. Different study designs included: randomised parallel $(n=3)$, randomised crossover $(n=1)$, and single intervention $(n=3)$. Interventions ranged in duration from 4 to 16 weeks. Sample sizes ranged from 24 to 107 and were heterogeneous in nature. For example, four samples comprised healthy participants, one comprised obese women with hyperlipidaemia, one comprised children and adolescents with hyperlipidaemia, and one specifically recruited people with overweight and obesity.

Three studies compared hazelnut consumption to a no-nut control group $[39,47,50]$, and two compared the consumption of different forms of hazelnuts $[39,49]$. None of these studies reported significant differences between treatments. Similarly, two single intervention studies reported no significant change in blood pressure following hazelnut consumption [53,56], and one single intervention reported a significant reduction in systolic blood pressure in the total cohort (combining Māori and European participants) [59].

\subsection{Glycaemia}

Nine studies examined glycaemia as an outcome, including one acute study (Table 8). The acute study measured $2 \mathrm{~h}$ incremental area under the curve (iAUC) for blood glucose in response to four breads containing no nuts, $30 \mathrm{~g}$ of finely sliced nuts, $30 \mathrm{~g}$ of defatted hazelnut flour, or $15 \mathrm{~g}$ of finely sliced nuts and $15 \mathrm{~g}$ of defatted hazelnut flour [40]. The iAUC for blood glucose was significantly lower for all hazelnut-containing breads compared to the no-nut bread.

The longer-term studies used a number of different indices to measure glycaemia. These included glycated haemoglobin (HbA1), fasting blood glucose (FBG), post-prandial blood glucose, fasting insulin, postprandial insulin, and the homeostasis model-insulin resistance (HOMA-IR).

Seven studies examined FBG concentrations. Only one study using a single intervention design showed a significant reduction in FBG [56].

Insulin concentrations were reported in four studies. Orem et al. reported that there was no significant difference in fasting insulin levels between the hazelnut-enriched diet and no nut control diet [57]. In addition, Adamo et al. reported that fasting insulin levels remained stable among those consuming $30 \mathrm{~g}$ of peeled hazelnut paste, $30 \mathrm{~g}$ of unpeeled hazelnut paste, or $30 \mathrm{~g}$ of peeled hazelnuts for breakfast for 2 weeks [36]. Actual changes in insulin were not presented, and no information on insulin levels in other groups receiving a cocoa snack, a combination of cocoa and $30 \mathrm{~g}$ peeled hazelnuts, and a no nut control was provided. Two other studies only assessed within-group differences and reported no significant changes in fasting or postprandial insulin concentrations $[51,56]$.

Only one study measured $\mathrm{HbA} 1 \mathrm{c}$, and it should be noted that the intervention was only for 30 days [51]. This study used a sequential design with a high carbohydrate diet and hazelnut diet among 19 people with type 2 diabetes. Between-group differences were not reported, but there was a significant reduction in $\mathrm{HbA} 1 \mathrm{c}$ in the hazelnut group.

Two studies assessed insulin resistance using HOMA-IR [36,57]. Adamo et al. did not report specific values, only commenting that HOMA-IR remained stable among those consuming $30 \mathrm{~g}$ of peeled hazelnut paste, $30 \mathrm{~g}$ of unpeeled hazelnut paste, or $30 \mathrm{~g}$ of peeled hazelnuts for breakfast for 2 weeks [36]. Orem reported non-significant differences in HOMA-IR between the hazelnut treatment and no-nut control in their sandwich model study [57].

Overall, the one acute study showed a reduction in iAUC for blood glucose with consumption of hazelnut in a carbohydrate-rich [40]. In studies with a longer intervention, only one of six studies reported lower FBG with hazelnut consumption. Three studies that assessed fasting and/or postprandial insulin showed no significant reductions with hazelnut consumption. One study reported reductions in $\mathrm{HbA1c}$ with hazelnut consumption among people with diabetes. Two studies that assessed HOMA-IR reported no significant differences with hazelnut consumption. 
Table 7. Effects of hazelnut consumption on blood pressure $(n=7)$

\begin{tabular}{|c|c|c|c|c|c|c|c|}
\hline Author, Year & Study Design & Participant Characteristics & Duration & Treatment & $\begin{array}{c}\mathrm{SBP} \\
\mathrm{mmHg}\end{array}$ & $\begin{array}{c}\text { DBP } \\
\mathrm{mmHg}\end{array}$ & Between Treatments \\
\hline \multirow{8}{*}{ Deon et al., 2018 [39] } & \multirow{8}{*}{$\begin{array}{l}\text { Randomised parallel } \\
3 \text { treatments }\end{array}$} & \multirow{8}{*}{$\begin{array}{l}66 \text { children and adolescents (35 M } 31 \text { F) } \\
\text { with hyperlipidaemia }\end{array}$} & \multirow{8}{*}{8 weeks } & \multirow{3}{*}{$\begin{array}{c}\text { (i) Baseline } \\
\text { Hazelnuts with skin }(0.43 \\
\text { g/kg }(15-30 \mathrm{~g} / \mathrm{d})) \\
\text { Change }^{1}\end{array}$} & 103.0 & 65.6 & \multirow{8}{*}{$\begin{array}{l}\text { No significant differences } \\
\text { in systolic blood pressure } \\
\text { or diastolic blood pressure } \\
\text { between the treatments. }\end{array}$} \\
\hline & & & & & 105.2 & 66.4 & \\
\hline & & & & & +2.2 & +0.8 & \\
\hline & & & & \multirow{3}{*}{$\begin{array}{c}\text { (ii) Baseline } \\
\text { Hazelnuts without skin } \\
(0.43 \mathrm{~g} / \mathrm{kg}(15-30 \mathrm{~g} / \mathrm{d})) \\
\text { Change }^{1}\end{array}$} & 102.8 & 65.1 & \\
\hline & & & & & 102.5 & 66.3 & \\
\hline & & & & & -0.3 & +1.2 & \\
\hline & & & & (iii) Baseline & 106.8 & 68.0 & \\
\hline & & & & Change $^{1}$ & +2.2 & & \\
\hline \multirow{2}{*}{ Di Renzo et al., 2019 [53] } & \multirow{2}{*}{$\begin{array}{l}\text { Single intervention } \\
\text { Pilot }\end{array}$} & \multirow{2}{*}{$\begin{array}{l}24 \text { (14 M, } 10 \text { F) } \\
\text { healthy }\end{array}$} & \multirow[b]{2}{*}{6 weeks } & Baseline $\ddagger$ & 116.5 & 73.0 & \multirow{2}{*}{$\mathrm{N} / \mathrm{A}$, single intervention. } \\
\hline & & & & (i) Hazelnuts $(40 \mathrm{~g} / \mathrm{d}){ }^{\ddagger}$ & 112.0 & 75.0 & \\
\hline \multirow{3}{*}{ Michels et al., 2018 [56] } & \multirow{3}{*}{ Single intervention } & \multirow{3}{*}{$\begin{array}{c}32(10 \mathrm{M}, 22 \mathrm{~F}) \text { healthy, non-frequent nut } \\
\text { consumers, Vit } \mathrm{E} \text { intake }<10 \mathrm{mg} \\
\text { a-tocopherol/d, no Vit E supplements in } \\
\text { previous } 12 \text { months }\end{array}$} & \multirow{3}{*}{16 weeks } & Baseline & 120 & 76.6 & \multirow{3}{*}{$\mathrm{N} / \mathrm{A}$, single intervention. } \\
\hline & & & & (i) Hazelnuts, dry roasted & 120 & 76.3 & \\
\hline & & & & $\begin{array}{l}(\sim 57 \mathrm{~g} / \mathrm{d}) \\
\text { Change }^{1}\end{array}$ & 0 & -0.3 & \\
\hline \multirow{8}{*}{ Tey et al., 2013 [47] } & \multirow{8}{*}{$\begin{array}{l}\text { Randomised } \\
\text { Parallel } \\
2 \text { treatments }\end{array}$} & \multirow{8}{*}{$\begin{array}{l}107(46 \mathrm{M}, 61 \mathrm{~F}) \\
\text { Overweight and obese individuals with a } \\
\mathrm{BMI} \geq 25 \mathrm{~kg} / \mathrm{m}^{2}\end{array}$} & \multirow{8}{*}{12 weeks } & Baseline & 128 & 75.3 & \multirow{8}{*}{$\begin{array}{l}\text { No significant difference in } \\
\text { systolic and diastolic blood } \\
\text { pressure between } \\
\text { the treatments. }\end{array}$} \\
\hline & & & & (i) Control (no hazelnuts) & 123 & 72.9 & \\
\hline & & & & Change $^{1}$ & $-5^{\text {a }}$ & $-2.4^{\mathrm{a}}$ & \\
\hline & & & & (ii) Hazelnuts ( $30 \mathrm{~g} / \mathrm{d})$ & 124 & 72.6 & \\
\hline & & & & Change $^{1}$ & -2 & -0.6 & \\
\hline & & & & Baseline & 124 & 76.3 & \\
\hline & & & & (iii) Hazelnuts $(60 \mathrm{~g} / \mathrm{d})$ & 121 & 73.3 & \\
\hline & & & & Change ${ }^{1}$ & $-3^{\mathrm{a}}$ & $-3.0^{\mathrm{a}}$ & \\
\hline \multirow{6}{*}{ Tey et al., 2015 [59] } & \multirow{6}{*}{ Single intervention } & \multirow{6}{*}{$\begin{array}{l}20 \text { Māori }(8 \mathrm{M}, 12 \mathrm{~F}) \text { and } 19(5 \mathrm{M}, 14 \mathrm{~F}) \\
\text { European aged above } 18 \text { years }\end{array}$} & \multirow{6}{*}{4 weeks } & Māori & 123.6 & & \multirow{6}{*}{$\begin{array}{l}\text { N/A, single intervention, } \\
\text { but there were no } \\
\text { significant differences in } \\
\text { systolic and diastolic blood } \\
\text { pressure between Māori } \\
\text { and Europeans. }\end{array}$} \\
\hline & & & & Baseline & $\begin{array}{l}123.6 \\
117.1\end{array}$ & 68.5 & \\
\hline & & & & $\begin{array}{l}\text { (i) Raw hazelnuts }(30 \mathrm{~g} / \mathrm{d})^{\wedge} \\
\text { Change }^{1}\end{array}$ & $\mathrm{~N} / \mathrm{R}$ & $\mathrm{N} / \mathrm{R}$ & \\
\hline & & & & & & & \\
\hline & & & & Baseline^ & $\begin{array}{l}120.1 \\
1184\end{array}$ & 65.5 & \\
\hline & & & & (i) Raw hazelnuts $(30 \mathrm{~g} / \mathrm{d})^{\wedge}$ & $\begin{array}{l}118.4 \\
N / R\end{array}$ & $\begin{array}{l}65.1 \\
\mathrm{~N} / \mathrm{R}\end{array}$ & \\
\hline
\end{tabular}


Table 7. Cont.

\begin{tabular}{|c|c|c|c|c|c|c|c|}
\hline Author, Year & Study Design & Participant Characteristics & Duration & Treatment & $\begin{array}{c}\text { SBP } \\
\mathrm{mmHg}\end{array}$ & $\begin{array}{c}\text { DBP } \\
\text { mmHg }\end{array}$ & Between Treatments \\
\hline \multirow{4}{*}{ Tey et al., 2017 [49] } & \multirow{4}{*}{$\begin{array}{l}\text { Randomised } \\
\text { Crossover } \\
2 \text { treatments }\end{array}$} & \multirow{4}{*}{$\begin{array}{l}72(24 \mathrm{M}, 48 \mathrm{~F}) \\
\text { Aged } 18 \text { years and above }\end{array}$} & \multirow{4}{*}{4 weeks } & $\begin{array}{c}\text { Baseline } \\
\text { (i) Raw hazelnuts }(30 \mathrm{~g} / \mathrm{d}) \\
\text { Change }^{1}\end{array}$ & $\begin{array}{c}124 \\
122 \\
-2.0^{\text {a }}\end{array}$ & $\begin{array}{l}73.5 \\
72.7 \\
-0.8\end{array}$ & \multirow{4}{*}{$\begin{array}{c}\text { No significant differences } \\
\text { in systolic blood pressure } \\
\text { between the treatments. } \\
\text { There was a tendency that } \\
\text { diastolic blood pressure } \\
\text { was lower after consuming } \\
\text { dry roasted and lightly } \\
\text { salted hazelnuts. }\end{array}$} \\
\hline & & & & \multirow{3}{*}{$\begin{array}{c}\text { Baseline } \\
\text { (ii) Dry roasted, lightly } \\
\text { salted hazelnuts }(30 \mathrm{~g} / \mathrm{d}) \\
\text { Change }^{1}\end{array}$} & 124 & 73.5 & \\
\hline & & & & & 121.1 & 71.5 & \\
\hline & & & & & $-2.9^{\mathrm{b}}$ & $-2.0^{\mathrm{b}}$ & \\
\hline \multirow{12}{*}{ Yilmaz et al., 2019 [50] } & \multirow{12}{*}{$\begin{array}{l}\text { Randomised } \\
\text { Parallel } \\
4 \text { treatments }\end{array}$} & \multirow{12}{*}{$\begin{array}{c}37(0 \mathrm{M}, 37 \mathrm{~F}) \\
\text { Hyperlipidaemia, Obese }\end{array}$} & \multirow{12}{*}{6 weeks } & \multirow{3}{*}{$\begin{array}{l}\text { Baseline } \\
\text { (i) Hazelnuts }(50 \mathrm{~g} / \mathrm{d}) \text { and } \\
\text { cardioprotective diet } \\
\text { Change }^{1}\end{array}$} & 121.7 & 77.2 & \multirow{12}{*}{$\begin{array}{l}\text { No significant difference in } \\
\text { systolic and diastolic blood } \\
\text { pressure between } \\
\text { the treatments. }\end{array}$} \\
\hline & & & & & 121.1 & 75.6 & \\
\hline & & & & & -0.6 & -1.7 & \\
\hline & & & & \multirow{3}{*}{$\begin{array}{c}\text { Baseline } \\
\text { (ii) Raisins }(50 \mathrm{~g} / \mathrm{d}) \text { and } \\
\text { cardioprotective diet } \\
\text { Change }^{1}\end{array}$} & 123.3 & 76.7 & \\
\hline & & & & & 119.4 & 76.7 & \\
\hline & & & & & -3.9 & 0.0 & \\
\hline & & & & \multirow{3}{*}{$\begin{array}{c}\text { Baseline } \\
\text { (iii) Hazelnuts }(50 \mathrm{~g} / \mathrm{d}) \\
\text { and Raisins }(50 \mathrm{~g} / \mathrm{d}) \text { and } \\
\text { cardioprotective diet } \\
\text { Change }^{1}\end{array}$} & 123.6 & 79.7 & \\
\hline & & & & & 115.6 & 75.6 & \\
\hline & & & & & $-8.0^{\mathrm{a}}$ & -4.1 & \\
\hline & & & & \multirow{3}{*}{$\begin{array}{c}\text { Baseline } \\
\text { (iv) Control } \\
\text { (Cardioprotective diet) } \\
\text { Change }^{1}\end{array}$} & 126.0 & 80.5 & \\
\hline & & & & & 122.0 & 77.5 & \\
\hline & & & & & -4.0 & -3.0 & \\
\hline
\end{tabular}

Abbreviations used: DBP, diastolic blood pressure; F, female; M, male; N/A, not applicable; SBP, systolic blood pressure. All values are arithmetic means unless otherwise stated ${ }^{1}$ Change (within-group) = Post-treatment value minus Pre-treatment value (i.e., baseline); ${ }^{\mathrm{a}} p<0.05 ;{ }^{\mathrm{b}} p<0.01$; only for those which reported within-group change. ${ }^{\wedge}$ Geometric mean. $\ddagger$ Median. 
Table 8. Effects of hazelnut consumption on glycaemic outcomes $(n=9)$.

\begin{tabular}{|c|c|c|c|c|c|}
\hline Author, Year & Study Design & Participant Characteristics & Duration & Treatment & Outcome Measurements: Results \\
\hline & & & Acute stu & & \\
\hline \multirow{4}{*}{ Devi et al., 2016 [40] } & \multirow{4}{*}{$\begin{array}{l}\text { Randomised crossover } \\
\quad 4 \text { treatments }\end{array}$} & \multirow{4}{*}{32 (11 M 21 F) healthy } & \multirow{4}{*}{ Acute $2 \mathrm{~h}$} & $\begin{array}{l}\text { (i) Bread containing } 30 \mathrm{~g} \text { finely sliced } \\
\text { hazelnuts per } 120 \mathrm{~g}\end{array}$ & $\begin{array}{l}2 \mathrm{~h} \text { iAUC for blood glucose } \\
\text { (i) } 152 \mathrm{mmol} / \mathrm{L} \cdot \mathrm{min}\end{array}$ \\
\hline & & & & $\begin{array}{l}\text { (ii) Bread containing } 30 \mathrm{~g} \text { defatted hazelnut } \\
\text { flour per } 120 \mathrm{~g}\end{array}$ & (ii) $137 \mathrm{mmol} / \mathrm{L} \cdot \min$ \\
\hline & & & & $\begin{array}{l}\text { (iii) Bread containing } 15 \mathrm{~g} \text { finely sliced } \\
\text { hazelnuts and } 15 \mathrm{~g} \text { defatted hazelnut flour per } \\
120 \mathrm{~g}\end{array}$ & (iii) $154 \mathrm{mmol} / \mathrm{L} \cdot \min$ \\
\hline & & & & (iv) Control white bread with no nuts & $\begin{array}{l}\text { (iv) } 179 \mathrm{mmol} / \mathrm{L} \cdot \min \\
\text { All hazelnut breads had a lower iAUC compared } \\
\text { to the control bread (all } p<0.001) \text {. There were no } \\
\text { significant differences between breads. }\end{array}$ \\
\hline \multirow{6}{*}{ Adamo et al., 2017 [36] } & & & Chronic st & & \\
\hline & \multirow{5}{*}{$\begin{array}{l}\text { Randomised parallel } \\
6 \text { treatments }\end{array}$} & \multirow{5}{*}{$\begin{array}{l}61(31 \mathrm{M}, 30 \mathrm{~F}) \\
\text { Healthy BMI }\end{array}$} & \multirow{5}{*}{2 weeks } & $\begin{array}{c}\text { Breakfasts including: } \\
\text { (i) } 30 \text { g peeled hazelnut paste }\end{array}$ & \multirow{5}{*}{$\begin{array}{l}\text { Insulin and HOMA-IR } \\
\text { Insulin and HOMA-IR remained stable in those } \\
\text { consuming the hazelnut-only enriched breakfasts } \\
\text { Actual data was not presented. }\end{array}$} \\
\hline & & & & (ii) $30 \mathrm{~g}$ unpeeled hazelnut paste & \\
\hline & & & & (iii) snack with $30 \mathrm{~g}$ peeled hazelnut paste & \\
\hline & & & & $\begin{array}{l}\text { (v) Snack with } 30 \mathrm{~g} / \mathrm{d} \text { peeled hazelnut paste } \\
\text { and } 2.5 \mathrm{~g} \text { cocoa powder }\end{array}$ & \\
\hline & & & & $\begin{array}{l}\text { (vi) no snack control group } \\
\text { N.B. Data was only presented for treatments vs. } \\
\text { control i.e., no other between-group comparisons } \\
\text { were reported }\end{array}$ & \\
\hline
\end{tabular}


Table 8. Cont.

Author, Year Study Design
Participant Characteristics

19 (5 M, 14 F)

Type 2 diabetics
Duration

Treatmen

Outcome Measurements: Results

(i) High $\mathrm{CHO} \operatorname{diet}(60 \% \mathrm{CHO}, 25 \%$ fat):

$$
\mathrm{HbA1c}
$$

Baseline: $8.1 \%$

End: $7.8 \%$

Change: $-0.3 \%$

$$
\text { FBG }
$$

Baseline6.92 mmol/L

End: $6.94 \mathrm{mmol} / \mathrm{L}$

Change: $+0.02 \mathrm{mmol} / \mathrm{L}$

$$
\text { PPBG }
$$

Baseline $9.16 \mathrm{mmol} / \mathrm{L}$

End: $8.49 \mathrm{mmol} / \mathrm{L}$

$$
\text { periods }
$$

30 days

Between-group analysis NR.

Fasting insulin

Baseline: $86.4 \mathrm{pmol} / \mathrm{L}$

End: $72.6 \mathrm{pmol} / \mathrm{L}$

Change: $-13.4 \mathrm{pmol} / \mathrm{L}$

$$
\text { PP insulin }
$$

Baseline $249.0 \mathrm{pmol} / \mathrm{L}$

End: $196.8 \mathrm{pmol} / \mathrm{L}$

Change: $-52.2 \mathrm{pmol} / \mathrm{L}$ 
Table 8. Cont.

Author, Year Study Design Participant Characteristics

Duration

Treatment

Outcome Measurements: Results

(ii) Hazelnut diet $(40 \% \mathrm{CHO}, 45 \%$ fat, amount

of hazelnuts NR)

$\mathrm{HbA} 1 \mathrm{c}$

Baseline: $8.3 \%$

End: $7.2 \%$

Change: $-1.1 \%$ a

FBG:

Baseline: $7.28 \mathrm{mmol} / \mathrm{L}$

End: $7.28 \mathrm{mmol} / \mathrm{L}$

Change: $0.00 \mathrm{mmol} / \mathrm{L}$

PPBC

Baseline: $8.37 \mathrm{mmol} / \mathrm{L}$

End: $8.28 \mathrm{mmol} / \mathrm{L}$

Change: $-0.09 \mathrm{mmol} / \mathrm{L}$

Fasting insulin

Baseline: $78.0 \mathrm{pmol} / \mathrm{L}$

End:97.2 pmol/L

Change: $+19.2 \mathrm{pmol} / \mathrm{L}$

PP insulin

Baseline: $223.2 \mathrm{pmol} / \mathrm{L}$

End: $225.0 \mathrm{pmol} / \mathrm{L}$

Change: $+1.8 \mathrm{pmol} / \mathrm{L}$

(i) Control: No hazelnuts

$$
\text { FBG }
$$

Baseline: $8.69 \mathrm{mmol} / \mathrm{L}$

End: $8.97 \mathrm{mmol} / \mathrm{L}$

Change: $+0.28 \mathrm{mmol} / \mathrm{L}$

Randomised parallel 2 treatments
50 (16 M, 34 F) participants with type 2 diabetes
8 weeks

(ii) $10 \%$ of total energy hazelnuts FBG

Baseline: $8.10 \mathrm{mmol} / \mathrm{L}$

End: $8.04 \mathrm{mmol} / \mathrm{L}$

Change: $-0.06 \mathrm{mmol} / \mathrm{L}$
Fasting blood glucose

There were no significant differences in fasting blood glucose concentrations 
Table 8. Cont.

\begin{tabular}{|c|c|c|c|c|c|}
\hline Author, Year & Study Design & Participant Characteristics & Duration & Treatment & Outcome Measurements: Results \\
\hline \multirow{2}{*}{ Michels et al., 2018 [56] } & \multirow{2}{*}{ Single intervention } & \multirow{2}{*}{$\begin{array}{l}32(10 \mathrm{M}, 22 \mathrm{~F} \mathrm{~F}) \text { healthy, } \\
\text { non-frequent nut consumers, } \\
\text { Vit } \mathrm{E} \text { intake }<10 \mathrm{mg} \\
\text { a-tocopherol/d, no Vit } \mathrm{E} \\
\text { supplements in previous } \\
12 \text { months }\end{array}$} & \multirow{2}{*}{16 weeks } & $\begin{array}{c}\text { (i) Baseline } \\
\text { FBG: } 5.67 \mathrm{mmol} / \mathrm{L} \\
\text { Fasting insulin: } 48.6 \mathrm{pmol} / \mathrm{L}\end{array}$ & \multirow{2}{*}{$\begin{array}{l}\text { Significant reduction in plasma FBG }(-3.4 \% \text {, } \\
p=0.03) \text { after } 16 \text { weeks consuming } 57 \mathrm{~g} / \text { day } \\
\text { hazelnuts. There was no significant change in } \\
\text { fasting insulin. }\end{array}$} \\
\hline & & & & $\begin{array}{c}\text { (ii) Hazelnuts, dry roasted ( } \sim 57 \mathrm{~g} / \text { day) } \\
\text { FBG: } 5.5 \mathrm{mmol} / \mathrm{L} \\
\text { Fasting insulin: } 49.8 \mathrm{pmol} / \mathrm{L}\end{array}$ & \\
\hline \multirow{3}{*}{ Orem et al., 2013 [57] } & \multirow{3}{*}{$\begin{array}{l}\text { Double control sandwich } \\
\text { model intervention }\end{array}$} & \multirow{3}{*}{$\begin{array}{l}21(18 \mathrm{M}, 3 \mathrm{~F}) \\
\text { Hyper-cholesterolaemic }\end{array}$} & \multirow{3}{*}{4 weeks } & $\begin{array}{l}\text { (i) } 4 \text { week no-nut (Control I) diet } \\
\text { FBG: } 5.22 \mathrm{mmol} / \mathrm{L} \\
\text { Fasting insulin: } 42.6 \mathrm{pmol} / \mathrm{L} \\
\text { HOMA-IR: } 1.69\end{array}$ & \multirow{3}{*}{$\begin{array}{l}\text { There was no significant difference in FBG, fasting } \\
\text { insulin, or HOMA-IR between treatments. }\end{array}$} \\
\hline & & & & $\begin{array}{l}\text { (ii) 4-week hazelnut-enriched diet (49-86 g/d } \\
\text { (18-20\% TER)) } \\
\text { FBG: } 5.11 \mathrm{mmol} / \mathrm{L}, \Delta:-1.52 \% \\
\text { Fasting insulin: } 45.6 \mathrm{pmol} / \mathrm{L}, \Delta:+14.7 \% \\
\text { HOMA-IR: } 1.78, \Delta:+13.1 \%\end{array}$ & \\
\hline & & & & $\begin{array}{c}\text { (iii) 4-week no-nut (Control II) diet } \\
\text { FBG: } 4.89 \mathrm{mmol} / \mathrm{L}, \Delta:-3.51 \% \\
\text { Fasting insulin: } 37.8 \mathrm{pmol} / \mathrm{L}, \Delta:-11.9 \% \\
\text { HOMA-IR: } 1.39, \Delta:-12.7 \%\end{array}$ & \\
\hline \multirow[t]{2}{*}{ Santi et al., 2017 [58] } & \multirow[t]{2}{*}{$\begin{array}{l}\text { Double control sandwich } \\
\text { model intervention }\end{array}$} & \multirow{2}{*}{$\begin{array}{c}24(14 \mathrm{M}, 10 \mathrm{~F}) \\
\text { Healthy } \\
\text { BMI }>19 \mathrm{~kg} / \mathrm{m}^{2},<30 \mathrm{~kg} / \mathrm{m}^{2}\end{array}$} & \multirow[t]{2}{*}{6 weeks } & $\begin{array}{l}\text { (ii) 6-week } 40 \mathrm{~g} \text { raw hazelnut } \\
\text { FBG: } 4.76 \mathrm{mmol} / \mathrm{L}\end{array}$ & \multirow[t]{2}{*}{$\begin{array}{c}\text { There was no significant difference in FBG } \\
\text { between treatments. }\end{array}$} \\
\hline & & & & $\begin{array}{c}\text { (iii) 6-week 'standard' diet 'washout' } \\
\text { FBG: } 4.77 \mathrm{mmol} / \mathrm{L}\end{array}$ & \\
\hline \multirow{2}{*}{ Tey et al., 2017 [49] } & \multirow{2}{*}{$\begin{array}{l}\text { Randomised } \\
\text { Crossover } \\
2 \text { treatments }\end{array}$} & \multirow{2}{*}{$\begin{array}{l}72(24 \mathrm{M}, 48 \mathrm{~F}) \\
\text { Aged } 18 \text { years and above }\end{array}$} & \multirow{2}{*}{4 weeks } & $\begin{array}{c}\text { Fasting blood glucose } \\
\text { Baseline: } 4.82 \mathrm{mmol} / \mathrm{L} \\
\text { (i) Raw hazelnuts }(30 \mathrm{~g} / \mathrm{d}): 4.80 \mathrm{mmol} / \mathrm{L} \\
\text { Change: }-0.02 \mathrm{mmol} / \mathrm{L}\end{array}$ & \multirow{2}{*}{$\begin{array}{l}\text { There was no significant difference in fasting } \\
\text { blood glucose between the treatments. }\end{array}$} \\
\hline & & & & $\begin{array}{l}\text { Baseline: } 4.82 \mathrm{mmol} / \mathrm{L} \\
\text { (ii) Dry roasted, lightly salted hazelnuts } \\
(30 \mathrm{~g} / \mathrm{d}): 4.81 \mathrm{mmol} / \mathrm{L} \\
\text { Change: }-0.01 \mathrm{mmol} / \mathrm{L}\end{array}$ & \\
\hline
\end{tabular}


Table 8. Cont.

Author, Year Study Design
Participant Characteristics
$37(0 \mathrm{M}, 37 \mathrm{~F})$

Hyperlipidaemia, Obese
Duration

6 weeks

\section{Treatment}

Fasting blood glucose

Baseline: $5.23 \mathrm{mmol} / \mathrm{L}$

(i) Hazelnuts $(50 \mathrm{~g} / \mathrm{d}): 5.18 \mathrm{mmol} / \mathrm{L}$

Change: $-0.05 \mathrm{mmol} / \mathrm{L}$

Baseline: $5.16 \mathrm{mmol} / \mathrm{L}$

(ii) Raisins (50 g/d): $5.64 \mathrm{mmol} / \mathrm{L}$

Change: $+0.48 \mathrm{mmol} / \mathrm{L}$

2019 [50] $\quad$ Parallel

Parallel (iii) Hazelnuts (50 g/d) and Raisins ( $50 \mathrm{~g} / \mathrm{d}$ ):

$$
5.17 \mathrm{mmol} / \mathrm{L}
$$

Change: $-0.16 \mathrm{mmol} / \mathrm{L}$

Baseline: $5.26 \mathrm{mmol} / \mathrm{L}$

(iv) Control (Cardioprotective diet):

$$
5.47 \mathrm{mmol} / \mathrm{L}
$$

Change: $+0.21 \mathrm{mmol} / \mathrm{L}$

To convert mmol/L blood glucose to mg/dL, multiply mmol/L by 18. Abbreviations used: F, female; FBG, fasting blood glucose; HbA1c, glycated haemoglobin; HOMA-IR, homeostasis model-insulin resistance; iAUC, incremental area under the curve; $\mathrm{M}$, male; NR, not reported; PP, postprandial; PPBG, postprandial blood glucose; TER, total energy requirement. All

values are arithmetic means unless otherwise stated. ${ }^{a} p<0.05$ only for those which reported within-group change.

There was no significant difference in fasting blood glucose between the treatments. 


\subsection{Inflammation, Oxidation, and Endothelial Function}

Sixteen studies have examined the effects of hazelnut consumption on antioxidant status and/or markers of inflammation, oxidative stress, and/or endothelial function (Table 9).

Nine studies assessed antioxidant status, with two studies reporting upregulation in the expression of genes involved in antioxidant and/or anti-inflammator pathways with hazelnut consumption [52,53]. A further three single intervention studies reported increased antioxidant markers [54,56,60]. Michels et al. reported improvements in some but not all outcomes [56]. Two studies reported no significant differences in alpha-tocopherol after consuming different forms of hazelnuts [43,49], although there was evidence of increases from baseline. A further three studies reported mixed results, with one reporting positive results [57] and two showing no differences between groups [37,47].

One acute study [41] and one chronic study (4 weeks) [52] reported a reduction in oxidised LDL after consuming $40 \mathrm{~g}$ of hazelnuts, compared to meals without nuts. A further single intervention reported significant reductions in oxidised LDL compared to baseline [60]. Conversely, there are mixed results when nut interventions are compared to no nut controls. Orem et al. reported significant reductions on oxidised LDL after consuming a hazelnut enriched diet. A further single intervention reported significant decreases in plasma malondialdehyde (MDA) [54]. Conversely, Guaraldi (2018) showed no significant differences in oxidised LDL, DNA strand breaks, and $\mathrm{H}_{2} \mathrm{O}_{2}$ DNA damage, while formamidopyrimidine DNA glycosylase (FPG)-sensitive sites in PBMCs were reduced significantly when hazelnut consumption was compared to a no nut control.

Seven studies looked at the effects of hazelnut consumption on inflammatory markers such as CRP and interleukin-6, with six studies (three RCTs and three single intervention studies) reporting no improvement in inflammatory markers $[36,37,47,56,59,60]$ and one reporting a significant reduction in CRP [57].

One study reported significant increases in peak systolic velocity (PSV) with hazelnut consumption compared to the control group [36]. Mercanligil reported no significant differences in endothelial function measured by doppler ultrasound [55], whereas Orem showed significant improvements [57]. Two RCTs assessed intracellular adhesion molecule1 (ICAM-1) and vascular adhesion molecule-1 (VCAM-1) [47,57]. Orem showed significant improvements in both markers with 49 to $86 \mathrm{~g} / \mathrm{d}$ of hazelnuts among people with hypercholesterolaemia [57], whereas Tey et al. showed no significant differences with 30 to $60 \mathrm{~g} / \mathrm{d}$ of hazelnuts among people with overweight and obesity [47]. 
Table 9. Effects of hazelnut consumption on antioxidant, oxidative stress, inflammatory markers, and endothelial function $(n=16)$.

\begin{tabular}{|c|c|c|c|c|c|}
\hline Author, Year & Study Design & $\begin{array}{c}\text { Participant } \\
\text { Characteristics }\end{array}$ & Duration & Treatments & Outcome Measurements: Results ${ }^{1}$ \\
\hline \multicolumn{6}{|c|}{ Acute study } \\
\hline Di Renzo et al., 2017 [41] & $\begin{array}{c}\text { Randomised crossover } \\
2 \text { treatments }\end{array}$ & $\begin{array}{c}22 \\
\text { healthy } \\
\text { BMI } \geq 19 \mathrm{~kg} / \mathrm{m}^{2} \\
\mathrm{BMI}<30 \mathrm{~kg} / \mathrm{m}^{2}\end{array}$ & $3 \mathrm{~h}$ & $\begin{array}{l}\text { (i) A high-fat McDonald's meal } \\
\text { (ii) A high-fat McDonald's meal with } 40 \mathrm{~g} \\
\text { of hazelnuts }\end{array}$ & $\begin{array}{l}\text { Oxidised LDL using ELISA kits } \\
\text { (i) Levels increased significantly by } 18 \% \text { from fasting to after } \\
\text { the McDonald's meal a (ii) No significant difference in levels } \\
\text { for the McDonald's meal with } 40 \mathrm{~g} \text { of hazelnuts } \\
\text { Levels were significantly lower after the McDonald's meal } \\
\text { with } 40 \mathrm{~g} \text { of hazelnuts compared to the McDonald's meal } \\
\qquad(-24.43 \%, p<0.05) \\
\text { N.B. Actual baseline and end of study values NR }\end{array}$ \\
\hline \multicolumn{6}{|c|}{ Chronic studies } \\
\hline Adamo et al., 2017 [36] & $\begin{array}{l}\text { Randomised parallel } \\
6 \text { treatments }\end{array}$ & $\begin{array}{c}61(31 \mathrm{M}, 30 \mathrm{~F}) \\
\text { Healthy BMI }\end{array}$ & 2-weeks & $\begin{array}{l}\text { Breakfasts including: } \\
\text { (i) } 30 \mathrm{~g} / \mathrm{d} \text { peeled hazelnut paste } \\
\text { (ii) } 30 \mathrm{~g} / \mathrm{d} \text { unpeeled hazelnut paste } \\
\text { (iii) snack with } 30 \mathrm{~g} / \mathrm{d} \text { peeled hazelnut paste } \\
\text { (iv) snack with } 2.5 \mathrm{~g} / \mathrm{d} \text { cocoa powder } \\
\text { (v) Snack with } 30 \mathrm{~g} / \mathrm{d} \text { peeled hazelnut paste } \\
\text { and } 2.5 \mathrm{~g} / \mathrm{d} \text { cocoa powder } \\
\text { (vi) no snack control group }\end{array}$ & $\begin{array}{r}\text { Peak systolic velocities (PSV), using Doppler ultrasound, at } \\
\text { rest vs. control } \\
\text { (i) Change: }+80.5 \% \text { a } \\
\text { (ii) Change: }+16.9 \% \\
\text { (iii) Change: }+33.7 \% \\
\text { (iv) Change: }+31.5 \% \\
\text { (v) Change: }+26.4 \% \\
\text { Compared to the control group PSV at rest increased } \\
\text { significantly in the peeled hazelnut paste group }(57.8 \% \text {, } \\
p=0.04) \text {; the unpeeled hazelnut group ( } 56.9 \%, p=0.04) ; \text { the } \\
\text { snack with peeled hazelnut paste group }(95.1 \%, p=0.002) ; \\
\text { the peeled hazelnuts and cocoa powder group }(68.5 \% \text {, } \\
p=0.01 \text { ). No significant differences between the snack group } \\
\text { with } 2.5 \mathrm{~g} / \mathrm{d} \text { cocoa powder and control } \\
\text { Peak systolic velocities (PSV) after } 3 \text { min of occlusion: } \\
\text { (i) Change: }+102.7 \% \\
\text { (ii) Change: }+15.6 \% \\
\text { (iii) Change: }+60.7 \% \\
\text { (iv) Change: }-7.1 \% \\
\text { (v) Change: }+64.7 \% \\
\text { Compared to the control, there were significant increases in } \\
\text { the snack with } 30 \mathrm{~g} / \mathrm{d} \text { of peeled hazelnut }(67.3 \%, p=0.002) ; \\
\text { and in the snack with } 30 \mathrm{~g} / \mathrm{d} \text { peeled hazelnut paste and } 2.5 \mathrm{~g} \\
\text { cocoa powder group }(22.9 \%, p=0.04) \text {. }\end{array}$ \\
\hline
\end{tabular}


Table 9. Cont.

\begin{tabular}{|c|c|c|c|c|c|}
\hline Author, Year & Study Design & $\begin{array}{c}\text { Participant } \\
\text { Characteristics }\end{array}$ & Duration & Treatments & Outcome Measurements: Results ${ }^{1}$ \\
\hline & & & & & $\begin{array}{l}\text { Erythrocyte sedimentation rate and hs-CRP } \\
\text { No between-group differences for ESR or hs-CRP } \\
\text { Heart rate } \\
\text { No significant differences in heart rate } \\
\text { N.B. Data was only presented for treatments vs. control, } \\
\text { i.e., no other between-group comparisons were reported. } \\
\text { Actual follow-up values NR. Actual end of study values for } \\
\text { Erythrocyte sedimentation rate, hs-CRP, and heart rate NR }\end{array}$ \\
\hline
\end{tabular}


Table 9. Cont.

\begin{tabular}{|c|c|c|c|c|c|}
\hline Author, Year & Study Design & $\begin{array}{l}\text { Participant } \\
\text { Characteristics }\end{array}$ & Duration & Treatments & Outcome Measurements: Results ${ }^{1}$ \\
\hline Di Renzo et al., 2014 [52] & $\begin{array}{c}\text { Sequential intervention } \\
\text { periods } \\
2 \text { treatments }\end{array}$ & $\begin{array}{rl} & 24 \\
\mathrm{BMI} & 19 \mathrm{~kg} / \mathrm{m}^{2}\end{array}$ & 4 weeks & $\begin{array}{l}\text { (i) 4-week standard diet (Italian } \\
\text { Mediterranean diet) } \\
\text { (ii) 4-week standard diet with hazelnuts } \\
40 \mathrm{~g} / \mathrm{d}\end{array}$ & $\begin{array}{c}\text { Oxidised LDL using ELISA kits } \\
\text { (i) study end: } 40.38 \mathrm{U} / \mathrm{L} \\
\text { (ii) study end: } 36.99 \mathrm{U} / \mathrm{L}^{\mathrm{b}} \\
\text { Oxidised LDL was significantly lower after hazelnut diet } \\
\text { compared to after standard diet }(p<0.05) \text {. } \\
\text { Gene expression was assessed using Quantitative Real-Time } \\
\text { PCR (RT2 Profiler PCR assays } \\
\text { The following genes were upregulated after hazelnut } \\
\text { consumption }{ }^{\mathrm{a}} \text { : BNIP3, GPX2, GSR, HSPAIA, TTN, TXNRDI } \\
\text { The following genes were downregulated after hazelnut } \\
\text { consumption }{ }^{\mathrm{a}} \text { : CCL5, KRTI, MBL2, PRDX6, SODI }\end{array}$ \\
\hline Di Renzo et al., 2019 [53] & $\begin{array}{l}\text { Single intervention } \\
\text { Pilot }\end{array}$ & $\begin{array}{c}24(14 \mathrm{M}, 10 \mathrm{~F}) \\
\text { healthy }\end{array}$ & 6 weeks & (i) Hazelnuts 40 g/d & $\begin{array}{c}\text { Gene expression was assessed using Quantitative Real Time } \\
\text { PCR (RT2 Profiler PCR assays } \\
\text { There was significant upregulation in the following genes } \\
\text { after consuming hazelnuts a: } \\
\text { superoxide dismutase (SODI) and catalase (CAT), } \\
\text { macrophage migration inhibitory factor (MFI), peroxisome } \\
\text { proliferator-activated receptor gamma (PPAR } \gamma \text { ), vitamin D } \\
\text { receptor (VDR), methylenetetrahydrofolate reductase } \\
\text { (MTHFR), angiotensin I-converting enzyme (ACE) - all } \\
\text { involved in antioxidant and/or anti-inflammatory pathways } \\
\text { No significant change in the expression of the } \\
\text { following genes after consuming hazelnuts: } \\
\text { apolipoprotein E (APOE), interleukin } 6 \text { receptor (IL6R), } \\
\text { nuclear factor of kappa light polypeptide gene enhancer in } \\
\text { B-cell 1 (NFKB1), insulin-like growth } \\
\text { factor } 2 \text { receptor (IFG2R), upstream transcription factor } \\
\text { 1 (USF1) }\end{array}$ \\
\hline Durak et al., 1999 [54] & Single intervention & $\begin{array}{c}30(18 \mathrm{M}, 12 \mathrm{~F}) \\
\text { Healthy } \\
\text { Medical students }\end{array}$ & 1 month & (i) Hazelnuts (1 g/kg BW (68-69 g)) & $\begin{array}{l}\text { Antioxidant potential by measuring TBARS }(1 / \mathrm{nmol} / \mathrm{mL} \cdot \mathrm{h}) \text { : } \\
\text { (i) Baseline: } 0.09 \text {, Hazelnut: } 0.11, \Delta:+0.02^{\mathrm{c}} \\
\text { Plasma malondialdehyde quantified as tissue thiobarbituric } \\
\text { acid-reactive material }(\mathrm{nmol} / \mathrm{mL}): \\
\text { (i) Baseline: } 1.33 \text {, Hazelnuts: } 0.99, \Delta:-0.34^{\mathrm{c}}\end{array}$ \\
\hline
\end{tabular}


Table 9. Cont.

\begin{tabular}{|c|c|c|c|c|c|}
\hline Author, Year & Study Design & $\begin{array}{c}\text { Participant } \\
\text { Characteristics }\end{array}$ & Duration & Treatments & Outcome Measurements: Results ${ }^{1}$ \\
\hline Guaraldi et al., 2018 [42] & Parallel intervention & $\begin{array}{c}60 \text { children and } \\
\text { adolescents (mean } \\
\text { age } 11.6 \pm 2.6 \text { years) } \\
\text { with } \\
\text { hyperlipidaemia }\end{array}$ & 8 weeks & $\begin{array}{l}\text { (i) Control (No nuts) } \\
\text { (ii) Hazelnuts with skin }(15-30 \mathrm{~g} / \mathrm{d}) \\
\text { (iii) Hazelnuts without skin }(15-30 \mathrm{~g} / \mathrm{d})\end{array}$ & $\begin{array}{c}\text { DNA strand breaks using COMET assay } \\
\text { Using endonuclease buffer (\%DNA in tail) } \\
\text { (i) Baseline: } 17.44 \% \\
\text { End: } 13.65 \% \\
\text { Change: }-3.65 \% \text { a } \\
\text { (ii) Baseline: } 18.66 \% \\
\text { End: } 13.41 \% \\
\text { Change: }-5.25 \% \text { a } \\
\text { (iii) Baseline: } 19.70 \% \\
\text { End: } 16.00 \% \\
\text { Change: }-3.70 \% \text { a } \\
\text { No differences between treatments. } \\
\text { DNA strand breaks using phosphate buffer saline } \\
\text { (\%DNA in tail) } \\
\text { (i) Baseline: } 6.85 \% \\
\text { End: } 6.25 \% \\
\text { Change: }-0.60 \% \\
\text { (ii) Baseline: } 6.53 \% \\
\text { End: } 6.83 \% \\
\text { Change: }+0.30 \% \\
\text { (iii) Baseline: } 6.15 \% \\
\text { End: } 6.64 \% \\
\text { Change: }+0.49 \% \\
\text { No differences between treatments. } \\
\text { FPG-sensitive sites in PBMCs measured using the enzyme } \\
\text { formamidopyrimidine DNA glycosylase } \\
\text { (\% DNA in tail) } \\
\text { (i) Baseline: } 15.9 \% \\
\text { End: } 18.9 \% \\
\text { Change: }+3.0 \% \text { a } \\
\text { (ii) Baseline: } 14.7 \% \\
\text { End: } 10.5 \% \\
\text { Change: }-4.2 \% \text { b } \\
\text { (iii) Baseline: } 13.9 \% \\
\text { End: } 10.1 \% \\
\text { Change: }-3.8 \% \text { b }\end{array}$ \\
\hline
\end{tabular}


Table 9. Cont.

\begin{tabular}{|c|c|c|c|c|c|}
\hline Author, Year & Study Design & $\begin{array}{c}\text { Participant } \\
\text { Characteristics }\end{array}$ & Duration & Treatments & Outcome Measurements: Results ${ }^{1}$ \\
\hline & & & & & $\begin{array}{c}\text { Significant between-group differences ( } p=0.001 \text { ) between } \\
\text { the } 2 \text { hazelnut groups and the control group. } \\
\mathrm{H}_{2} \mathrm{O}_{2} \text {-induced DNA damage using COMET assay } \\
\text { (\% DNA in tail) } \\
\text { (i) Baseline: } 35.3 \% \\
\text { End: } 29.6 \% \\
\text { Change: }-5.7 \% \\
\text { (ii) Baseline: } 36.6 \% \\
\text { End: } 28.7 \% \\
\text { Change: }-7.9 \% \mathrm{~b} \\
\text { (iii) Baseline: } 37.4 \% \\
\text { End: } 32.0 \% \\
\text { Change: }-5.4 \% \\
\text { No significant differences between treatments } \\
\text { Oxidised LDL by ELISA } \\
\text { (i) Baseline: } 54.1 \mathrm{U} / \mathrm{L} \\
\text { End: } 55.1 \mathrm{U} / \mathrm{L} \\
\text { Change: } 1.0 \mathrm{U} / \mathrm{L} \\
\text { (ii) Baseline: } 54.5 \mathrm{U} / \mathrm{L} \\
\text { End: } 53.3 \mathrm{U} / \mathrm{L} \\
\text { Change: }-1.2 \mathrm{U} / \mathrm{L} \\
\text { (iii) Baseline: Not measured } \\
\text { End: Not measured } \\
\text { Change: Not measured } \\
\text { No significant differences between treatments }\end{array}$ \\
\hline Mercanligil et al., 2007 [55] & $\begin{array}{c}\text { Sequential intervention } \\
\text { periods } \\
2 \text { treatments }\end{array}$ & $\begin{array}{c}15(15 \mathrm{M}, 0 \mathrm{~F}) \\
\text { Hyper-cholesterolaemic }\end{array}$ & 4 weeks & $\begin{array}{l}\text { (i) Control LF, low cholesterol, high } \mathrm{CHO} \text { diet } \\
\text { (ii) Control + Hazelnuts ( } 40 \mathrm{~g} / \mathrm{d})\end{array}$ & $\begin{array}{l}\text { Vascular endothelium function by Doppler ultrasound } \\
\text { (i) Baseline: NR, Control: NR, Change: NR } \\
\text { (ii) Baseline: NR, Hazelnuts: NR, Change: NR } \\
\text { There were no significant differences in endothelial function } \\
\text { between the groups. }\end{array}$ \\
\hline
\end{tabular}


Table 9. Cont.

\begin{tabular}{|c|c|c|c|c|c|}
\hline Author, Year & Study Design & $\begin{array}{c}\text { Participant } \\
\text { Characteristics }\end{array}$ & Duration & Treatments & Outcome Measurements: Results ${ }^{1}$ \\
\hline Michels et al., 2018 [56] & Single intervention & $\begin{array}{c}32(10 \mathrm{M}, 22 \mathrm{~F} \mathrm{~F}) \\
\text { healthy, } \\
\text { non-frequent nut } \\
\text { consumers, Vit } \mathrm{E} \\
\text { intake }<10 \mathrm{mg} \\
\text { a-tocopherol/d, no } \\
\text { Vit E supplements in } \\
\text { previous } 12 \text { months }\end{array}$ & 16 weeks & $\begin{array}{c}\text { Baseline } \\
\text { (i) Hazelnuts, dry roasted }(\sim 57 \mathrm{~g} / \mathrm{d})\end{array}$ & 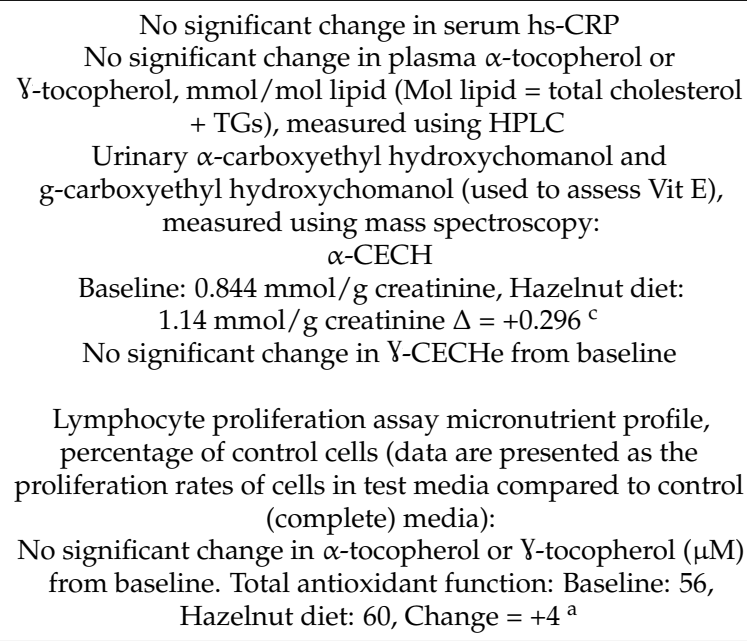 \\
\hline
\end{tabular}

(iii) Control II diet: $0.90 \mathrm{mg} / \mathrm{L}$, Change: $+71.1 \%$

There was a significant difference in Hs-CRP between (i) and

(ii), and between (ii) and (iii). There was no significant difference between (i) and (iii). 
Table 9. Cont.

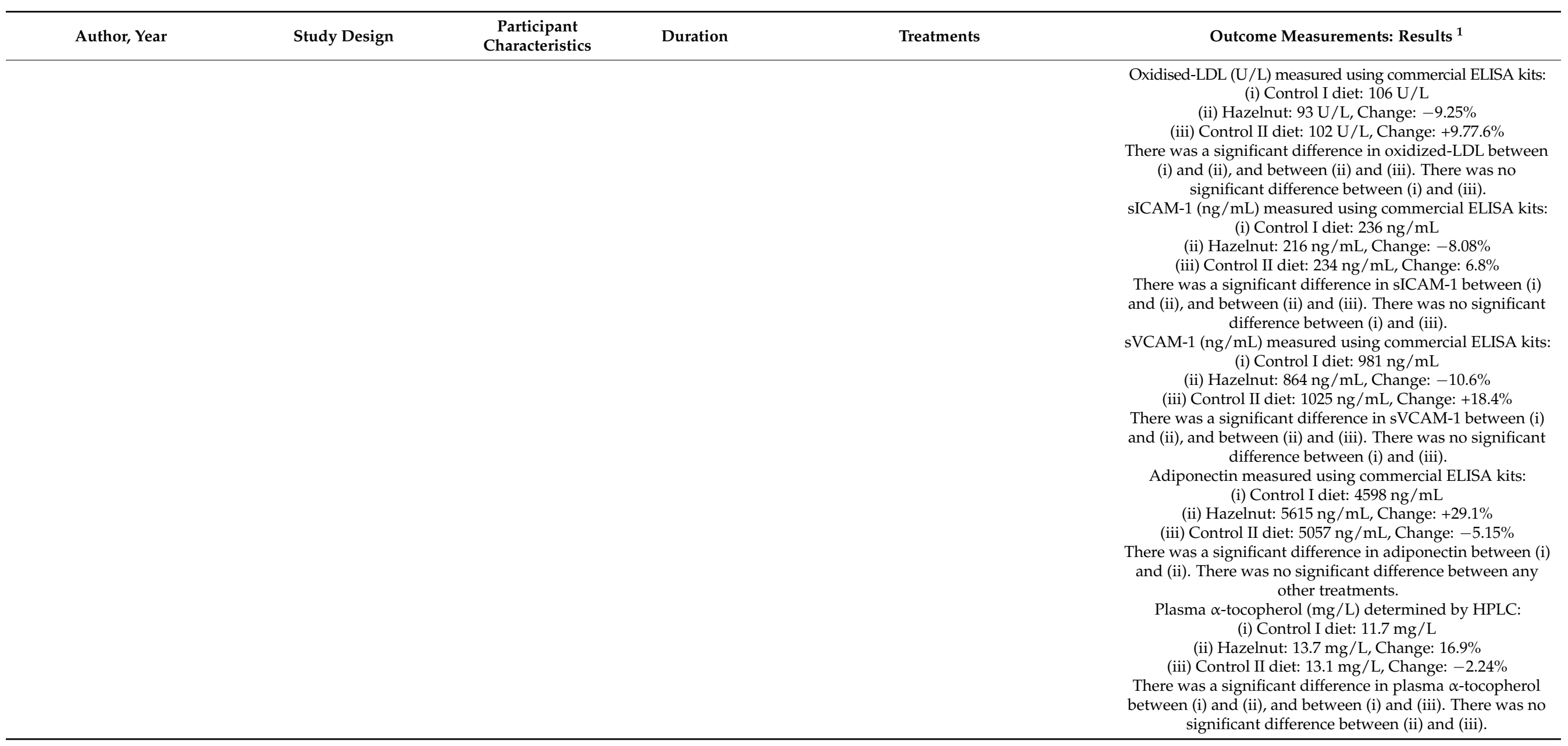


Table 9. Cont.

\begin{tabular}{|c|c|c|c|c|c|}
\hline Author, Year & Study Design & $\begin{array}{c}\text { Participant } \\
\text { Characteristics }\end{array}$ & Duration & Treatments & Outcome Measurements: Results ${ }^{1}$ \\
\hline & & & & & $\begin{array}{c}\alpha \text {-tocopherol in LDL, determined by HPLC } \\
\text { ( } \mu \mathrm{g} / \mathrm{mg} \text { LDL protein): } \\
\text { (i) Control I diet: } 4.71 \mu \mathrm{g} / \mathrm{mg} \\
\text { (ii) Hazelnut: } 5.76 \mu \mathrm{g} / \mathrm{mg} \text {, Change: } 24.5 \% \\
\text { (iii) Control II diet: } 4.41 \mu \mathrm{g} / \mathrm{mg} \text {, Change: }-22.3 \% \\
\text { There was a significant difference in } \alpha \text {-tocopherol in LDL } \\
\text { between (i) and (ii) and between (ii) and (iii). There was no } \\
\text { significant difference between (i) and (iii). } \\
\text { Vitamin B12 (pg/mL) measured by enzymatic methods: } \\
\text { (i) Control I diet: } 375 \mathrm{pg} / \mathrm{mL} \\
\text { (ii) Hazelnut: } 386 \mathrm{pg} / \mathrm{mL}, \mathrm{Change:}+2.94 \% \\
\text { (iii) Control II diet: } 334 \mathrm{pg} / \mathrm{mL}, \text { Change: }-13.8 \% \\
\text { There was a significant difference in vitamin B12 between (i) } \\
\text { and (iii), and between (ii) and (iii). There was no significant } \\
\text { difference between (i) and (ii). } \\
\text { Folic acid (ng/mL), measured by enzymatic methods: } \\
\text { (i) Control I diet: } 8.58 \text { ng } / \mathrm{mL} \\
\text { (ii) Hazelnut: } 9.08 \mathrm{ng} / \mathrm{mL}, \text { Change: }+6.24 \% \\
\text { (iii) Control II diet: } 8.04 \mathrm{ng} / \mathrm{mL}, \text { Change: }-11.3 \% \\
\text { There was a significant difference in folic acid between (ii) } \\
\text { and (iii). There was no significant difference between the } \\
\text { other treatments. } \\
\text { There was no significant difference in endothelin-1 } \\
\text { (fmol/mL) or homocysteine }(\mu \text { mol/L) across any of the } \\
\text { treatments, overall } p \text {-value } p=0.651 \text { and } \\
p=0.484 \text { respectively. } \\
\text { N.B. } p \text {-values for between-group differences NR. }\end{array}$ \\
\hline
\end{tabular}


Table 9. Cont.

\begin{tabular}{|c|c|c|c|c|c|}
\hline Author, Year & Study Design & $\begin{array}{c}\text { Participant } \\
\text { Characteristics }\end{array}$ & Duration & Treatments & Outcome Measurements: Results ${ }^{1}$ \\
\hline Santi et al., 2017 [58] & $\begin{array}{l}\text { Double control sandwich } \\
\text { model intervention }\end{array}$ & $\begin{array}{c}24(14 \mathrm{M}, 10 \mathrm{~F}) \\
\text { Healthy } \\
\text { BMI }>19 \mathrm{~kg} / \mathrm{m}^{2} \\
<30 \mathrm{~kg} / \mathrm{m}^{2}\end{array}$ & 6-weeks & $\begin{array}{l}\text { (i) 2-week 'standard' diet } \\
\text { (ii) 6-week raw hazelnut ( } 40 \mathrm{~g} / \mathrm{d} \text { ) } \\
\text { (iii) 6-week 'standard' diet 'washout' }\end{array}$ & $\begin{array}{c}\text { Uric acid (mg/dL) measured by uricase and } \\
\text { peroxidase reactions: } \\
\text { (i): } 4.66 \\
\text { (ii): } 4.31 \text {, Change: }-0.35 \\
\text { (iii): } 4.66 \text {, Change: }+0.35 \\
\text { (i) vs. (iii) } p=0.99 \\
\text { (ii) vs. (iii) } p=0.013 \\
\text { There was a significant } p=0.025 \\
\text { Serum creatinine (mg/dL) measured by creatinine } \\
\text { (i): } 0.94 \\
\text { amidohydrolase, sarcosine and peroxidase reactions: } \\
\text { (ii): } 0.93 \text {, Change: }-0.01 \\
\text { (iii): } 0.82 \text {, Change: }-0.11 \\
\text { (i) vs. (ii) } p=0.29 \\
\text { (i) vs. (iii) } p \leq 0.001 \\
\text { (ii) vs. (iii) } p=0.001 \\
\text { latticodehydrogenase reactions: } \\
\text { (i): } 30.09 \\
\text { (ii): } 35.22, \text { Change: }+5.13 \\
\text { (iii): } 31.52, \text { Change: }-3.70 \\
\text { (i) vs. (ii) } p=0.011 \\
\text { (i) vs. (iii) } p=0.065 \\
\text { (ii) vs. (iii) } p=0.99 \\
\text { Alanine aming by } \\
\text { Gamma-glutamyl transferase (GGT) (U) measured by } \\
\text { oxaloacetate decarboxylase, pyruvate oxidase and } \\
\text { peroxidase reactions: } \\
\text { (i): } 38.04 \\
\text { (ii): } 35.27 \text {, Change: }-2.77 \\
\text { (iii): } 36.26, \text { Change: }+0.99 \\
\text { (i) vs. (ii) } p=0.001 \\
\text { (i) vs. (iii) } p=0.31 \\
\text { (ii) vs. (iii) } p=0.16 \\
\text { There was no significant difference in AST, serum iron, } \\
\text { azotaemia, total bilirubin, Hb, WBCs, RBC, platelet count, or } \\
\text { total plasma protein content between any of the } \\
\text { treatment groups. }\end{array}$ \\
\hline
\end{tabular}


Table 9. Cont.

\begin{tabular}{|c|c|c|c|c|c|}
\hline Author, Year & Study Design & $\begin{array}{c}\text { Participant } \\
\text { Characteristics }\end{array}$ & Duration & Treatments & Outcome Measurements: Results ${ }^{1}$ \\
\hline Tey et al., 2011 [43] & $\begin{array}{l}\text { Randomised } \\
\text { Crossover } \\
3 \text { treatments }\end{array}$ & $\begin{array}{l}48(20 \mathrm{M}, 28 \mathrm{~F}) \\
\text { Mildly hyper- } \\
\text { cholesterolaemic }\end{array}$ & 4 weeks & $\begin{array}{l}\text { (i) Ground hazelnuts }(30 \mathrm{~g} / \mathrm{d}) \\
\text { (ii) Sliced hazelnuts }(30 \mathrm{~g} / \mathrm{d}) \\
\text { (iii) Whole hazelnuts }(30 \mathrm{~g} / \mathrm{d})\end{array}$ & $\begin{array}{l}\alpha \text {-tocopherol measured using HPLC (mmol/L): } \\
\text { (i) Baseline: } 33.1, \text { Ground: } 34.7 \text {, Change: }+1.6^{\mathrm{b}} \\
\text { (ii) Baseline: } 33.1 \text {, Sliced: } 34.2, \text { Change: }+1.1^{\mathrm{b}} \\
\text { (iii) Baseline: } 33.1 \text {, Whole: } 34.2 \text {, Change: }+1.1^{\mathrm{b}} \\
\text { There was no significant difference in } \alpha \text {-tocopherol between } \\
\text { different forms of nuts. }\end{array}$ \\
\hline Tey et al., 2013 [47] & $\begin{array}{l}\text { Randomised } \\
\text { Parallel } \\
3 \text { treatments }\end{array}$ & $\begin{array}{c}107(46 \mathrm{M}, 61 \mathrm{~F}) \\
\text { Overweight and } \\
\text { obese individuals } \\
\text { with a } \\
\text { BMI } \geq 25 \mathrm{~kg} / \mathrm{m}^{2}\end{array}$ & 12 weeks & $\begin{array}{l}\text { (i) Control group (no hazelnuts) } \\
\text { (ii) Hazelnuts }(30 \mathrm{~g} / \mathrm{d}) \\
\text { (iii) Hazelnuts }(60 \mathrm{~g} / \mathrm{d})\end{array}$ & 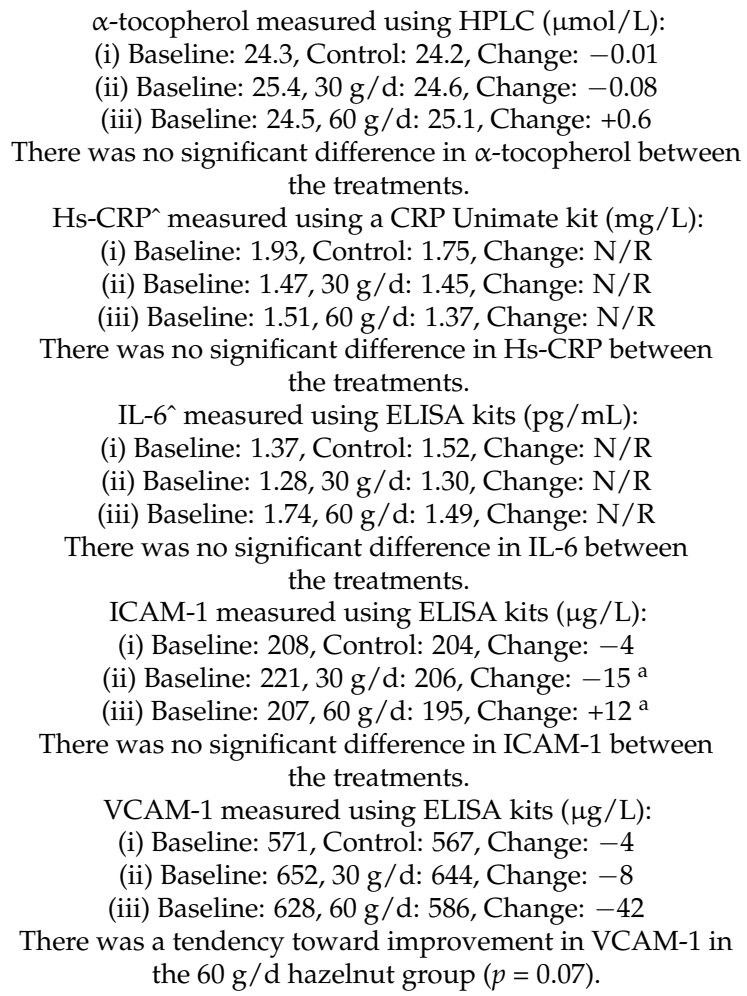 \\
\hline
\end{tabular}


Table 9. Cont.

\begin{tabular}{|c|c|c|c|c|c|}
\hline Author, Year & Study Design & $\begin{array}{c}\text { Participant } \\
\text { Characteristics }\end{array}$ & Duration & Treatments & Outcome Measurements: Results ${ }^{1}$ \\
\hline Tey et al., 2015 [59] & Single intervention & $\begin{array}{c}20 \text { Māori }(8 \mathrm{M}, 12 \mathrm{~F}) \\
\text { and } 19(5 \mathrm{M}, 14 \mathrm{~F}) \\
\text { European aged } \\
\text { above } 18 \text { years }\end{array}$ & 4 weeks & (i) Raw hazelnuts ( $30 \mathrm{~g} / \mathrm{d}$ ) & $\begin{array}{l}\text { Hs-CRP ^ measured using a CRP Unimate kit (mg/L): } \\
\text { Māori: (i) Baseline: 0.42, Hazelnuts: 0.70, Change: N/R } \\
\text { Europeans: (i) Baseline: } 0.69 \text {, Hazelnuts: 0.83, Change: N/R }\end{array}$ \\
\hline Tey et al., 2017 [49] & $\begin{array}{l}\text { Randomised } \\
\text { Crossover } \\
2 \text { treatments }\end{array}$ & $\begin{array}{l}72(24 \mathrm{M}, 48 \mathrm{~F}) \\
\text { Aged } 18 \text { years } \\
\text { and above }\end{array}$ & 4 weeks & $\begin{array}{l}\text { (i) Raw hazelnuts }(30 \mathrm{~g} / \mathrm{d}) \\
\text { (ii) Dry roasted, lightly salted hazelnuts } \\
(30 \mathrm{~g} / \mathrm{d})\end{array}$ & $\begin{array}{l}\alpha \text {-tocopherol measured using HPLC }(\mu \mathrm{mol} / \mathrm{L}) \text { : } \\
\text { (i) Baseline: } 30.2, \text { Raw: } 31.42, \text { Change: }+1.22 \mathrm{~b} \\
\text { (ii) Baseline: } 30.2, \text { Lightly salted: } 31.26 \text {, Change: }+1.06 \\
\text { There was no significant difference in } \alpha \text {-tocopherol between } \\
\text { the treatments. }\end{array}$ \\
\hline Yucesan et al., 2010 [60] & Single intervention & $\begin{array}{c}21(8 \mathrm{M}, 13 \mathrm{~F}) \\
\text { Normolipidaemic }\end{array}$ & 4 weeks & (i) Hazelnuts (1 g/kg BW (49-86 g)) & $\begin{array}{c}\alpha \text {-tocopherol in LDL ( } \mu \mathrm{g} / \mathrm{mg} \text { LDL protein), measured } \\
\text { using HPLC: } \\
\text { (i) Baseline: } 4.82, \text { Hazelnuts: } 5.35 \text {, Change: }+0.53^{\mathrm{a}} \\
\text { Oxidised LDL (U/L): } \\
\text { (i) Baseline: } 57.2 \text {, Hazelnut: } 48.2 \text {, Change: }-9.0^{\mathrm{b}} \\
\text { Hs-CRP (mg/dL), measured using } \\
\text { immunophrelometric method: } \\
\text { (i) Baseline: } 0.13 \text {, Hazelnut: } 0.11 \text {, Change: }-0.02 \\
\text { sVCAM-1 (ng/mL), measured using ELISA kits: } \\
\text { (i) Baseline: } 478, \text { Hazelnut: } 446 \text {, Change: }-32 \\
\text { Endothelin-1 (fmol/mL), measured using ELISA kits: } \\
\text { (i) Baseline: } 2.04, \text { Hazelnut: } 1.99 \text {, Change: }-0.05\end{array}$ \\
\hline
\end{tabular}




\subsection{Sensory Acceptance}

Seven studies have measured the effects of repeated consumption of hazelnuts on the desire to consume and overall liking using $100 \mathrm{~mm}$ or $150 \mathrm{~mm}$ visual analogue scales with exposure ranging from 5 to 84 days (Table 10). Both ratings remained stable over time, except for one dose-response study [47]. This study showed the desire to consume ratings increased over time with $30 \mathrm{~g} / \mathrm{d}$ of hazelnuts for 12 weeks, whereas the desire to consume and overall liking ratings decreased over time for the $60 \mathrm{~g} / \mathrm{d}$ groups.

Several studies compared different forms of hazelnuts $[40,44,48,49]$. Devi et al. incorporated different forms of hazelnuts into bread. Desire to eat and overall liking ratings from highest to lowest were: bread containing $30 \mathrm{~g}$ finely sliced hazelnuts, bread containing $15 \mathrm{~g}$ finely sliced hazelnuts, and $15 \mathrm{~g}$ of defatted hazelnut flour, control bread containing no nuts, and bread containing $30 \mathrm{~g}$ defatted hazelnut flour [40].

A further two studies reported desire to consume, and overall liking ratings were highest for whole hazelnuts, followed by sliced hazelnuts, and ground hazelnuts had the lowest ratings [44,48].

One study compared acceptance ratings for raw hazelnuts with dry roasted, lightly salted hazelnuts. Both forms of hazelnuts were equally liked [49].

One study compared isocaloric amounts of hazelnuts $(42 \mathrm{~g} / \mathrm{d})$ with chocolate $(50 \mathrm{~g} / \mathrm{d})$ and potato crisps $(50 \mathrm{~g} / \mathrm{d})$. The liking ratings for hazelnuts remained stable over time, whereas the ratings for both chocolate and potato crisps declined significantly [46]. 
Table 10. Dietary intervention trials investigating the effects of nut consumption on acceptance $(n=7)$.

\begin{tabular}{|c|c|c|c|c|c|}
\hline Author, Year & Study Design & Subjects & $\begin{array}{l}\text { Measurement; } \\
\text { Timepoint }\end{array}$ & Treatments; Number of Exposures & Results $^{1}$ \\
\hline \multirow[b]{2}{*}{ Devi et al., 2016 [40] } & \multirow[b]{2}{*}{$\begin{array}{l}\text { Randomised } \\
\text { Crossover } \\
4 \text { treatments }\end{array}$} & \multirow[b]{2}{*}{$\begin{array}{l}32(11 \mathrm{M} 21 \mathrm{~F}) \\
\text { healthy }\end{array}$} & $\begin{array}{l}\text { Desire to consume on a } 150 \mathrm{~mm} \text { VAS; Measured } \\
\text { daily during the exposure period }\end{array}$ & $\begin{array}{l}\text { (i) Bread containing } 30 \mathrm{~g} \text { finely sliced hazelnuts per } \\
120 \mathrm{~g} \text {; Exp. period }=5 \mathrm{~d} \\
\text { (ii) Bread containing } 30 \mathrm{~g} \text { defatted hazelnut flour } \\
\text { per } 120 \mathrm{~g} \text {; Exp. period }=5 \mathrm{~d} \\
\text { (iii) Bread containing } 15 \mathrm{~g} \text { finely sliced defatted } \\
\text { hazelnuts and } 15 \mathrm{~g} \text { hazelnut flour per } 120 \mathrm{~g} \text {; Exp. } \\
\text { period }=5 \mathrm{~d} \\
\text { (iv) Control white bread with no nuts; Exp. } \\
\text { period }=5 \mathrm{~d}\end{array}$ & $\begin{array}{l}\text { 5-day exposure period } \\
\text { (i) Stable: }{ }^{\mathrm{a}} \\
\text { (ii) Stable: }{ }^{\mathrm{c}} \\
\text { (iii) Stable: }{ }^{\mathrm{a}} \\
\text { (iv) Stable: }{ }^{\mathrm{b}}\end{array}$ \\
\hline & & & $\begin{array}{l}\text { Overall liking on a } 150 \mathrm{~mm} \text { VAS; } \\
\text { Measured daily during the exposure period }\end{array}$ & $\begin{array}{l}\text { (i) Bread containing } 30 \mathrm{~g} \text { finely sliced hazelnuts per } \\
120 \mathrm{~g} \text {; Exp. period }=5 \mathrm{~d} \\
\text { (ii) Bread containing } 30 \mathrm{~g} \text { defatted hazelnut flour } \\
\text { per } 120 \mathrm{~g} \text {; Exp. period }=5 \mathrm{~d} \\
\text { (iii) Bread containing } 15 \mathrm{~g} \text { of finely sliced defatted } \\
\text { hazelnuts and } 15 \mathrm{~g} \text { hazelnut flour per } 120 \mathrm{~g} \text {; Exp. } \\
\text { period }=5 \mathrm{~d} \\
\text { (iv) Control white bread with no nuts; Exp. } \\
\text { period }=5 \mathrm{~d}\end{array}$ & $\begin{array}{l}\text { 5-day exposure period } \\
\text { (i) Stable: }{ }^{\mathrm{a}} \\
\text { (ii) Stable: } \mathrm{c} \\
\text { (iii) Stable: }{ }^{\mathrm{b}} \\
\text { (iv) Stable: }{ }^{\mathrm{b}} \\
\text { Pre- vs. Post- } \\
\text { (i) No significant change: } 74.8^{\mathrm{b}} \text { vs. } 79.3^{\mathrm{b}} \\
\text { (ii) No significant change: } 46.5^{\mathrm{a}} \text { vs. } 41 . \mathrm{a}^{\mathrm{a}} \\
\text { (iii) Significant increase: } 53.4^{\mathrm{a}} \text { vs. } 66 . \mathrm{c}^{\mathrm{c}}(p<0.05) \\
\text { (iv) No significant change: } 44.5^{\mathrm{a}} \text { vs. } 46.5^{\mathrm{a}}\end{array}$ \\
\hline \multirow[b]{2}{*}{ Tey et al., 2011 [44] } & & \multirow[b]{2}{*}{$20 \mathrm{M}, 28 \mathrm{~F}$} & $\begin{array}{l}\text { Desire to consume on a } 150 \mathrm{~mm} \text { VAS; Measured } \\
\text { daily during the exposure period }\end{array}$ & $\begin{array}{l}\text { (i) Ground hazelnuts }(30 \mathrm{~g} / \mathrm{d}) \text {; Exp. period }=28 \mathrm{~d} \\
\text { (ii) Sliced hazelnuts }(30 \mathrm{~g} / \mathrm{d}) ; \text { Exp. period }=28 \mathrm{~d} \\
\text { (iii) Whole hazelnuts }(30 \mathrm{~g} / \mathrm{d}) \text {; Exp. period }=28 \mathrm{~d}\end{array}$ & $\begin{array}{l}\text { 28-day exposure period } \\
\text { (i) Stable: } 92.1^{\mathrm{a}} \\
\text { (ii) Stable: } 107.7^{\mathrm{b}} \\
\text { (iii) Stable: } 116.2^{\mathrm{b}}\end{array}$ \\
\hline & $\begin{array}{l}\text { Randomised } \\
\text { Crossover } \\
3 \text { treatments }\end{array}$ & & $\begin{array}{l}\text { Overall liking on a } 150 \mathrm{~mm} \text { VAS; } \\
\text { Measured daily during the exposure period and } \\
\text { at pre- and post-exposure }\end{array}$ & $\begin{array}{l}\text { (i) Ground hazelnuts }(30 \mathrm{~g} / \mathrm{d}) \text {; Exp. period }=28 \mathrm{~d} \\
\text { (ii) Sliced hazelnuts }(30 \mathrm{~g} / \mathrm{d}) ; \text { Exp. period }=28 \mathrm{~d} \\
\text { (iii) Whole hazelnuts }(30 \mathrm{~g} / \mathrm{d}) ; \text { Exp. period }=28 \mathrm{~d}\end{array}$ & $\begin{array}{l}\text { 28-day exposure period } \\
\text { (i) Stable: } 100.8^{\mathrm{a}} \\
\text { (ii) Stable: } 109.9^{\mathrm{b}} \\
\text { (iii) Stable: } 117.7^{\mathrm{b}} \\
\text { Pre- vs. Post- } \\
\text { (i) No significant change: } 92.8^{\mathrm{a}} \text { vs. } 87.4^{\mathrm{a}} \\
\text { (ii) No significant change: } 109.1^{\mathrm{b}} \text { vs. } 107.3^{\mathrm{b}} \\
\text { (iii) No significant change: } 113.7^{\mathrm{b}} \text { vs. } 110.2^{\mathrm{b}}\end{array}$ \\
\hline
\end{tabular}


Table 10. Cont.

\begin{tabular}{|c|c|c|c|c|c|}
\hline Author, Year & Study Design & Subjects & $\begin{array}{l}\text { Measurement; } \\
\text { Timepoint }\end{array}$ & Treatments; Number of Exposures & Results $^{1}$ \\
\hline \multirow[b]{2}{*}{ Tey et al., 2012 [46] } & \multirow[b]{2}{*}{$\begin{array}{l}\text { Randomised } \\
\text { Parallel } \\
4 \text { treatments }\end{array}$} & \multirow[b]{2}{*}{$55 \mathrm{M}, 63 \mathrm{~F}$} & $\begin{array}{l}\text { Desire to consume on a } 100 \mathrm{~mm} \text { VAS; Measured } \\
\text { daily during the exposure period }\end{array}$ & $\begin{array}{l}\text { (i) Hazelnuts }(42 \mathrm{~g} / \mathrm{d}) \text {; Exp. period }=84 \mathrm{~d} \\
\text { (ii) Chocolate }(50 \mathrm{~g} / \mathrm{d}) \text {; Exp. period }=84 \mathrm{~d} \\
\text { (iii) Potato crisps }(50 \mathrm{~g} / \mathrm{d}) ; \text { Exp. period }=84 \mathrm{~d}\end{array}$ & $\begin{array}{l}\text { 84-day exposure period } \\
\text { (i) Stable: } 60.9^{\mathrm{a}} \\
\text { (ii) Stable: } 64.9^{\mathrm{a}} \\
\text { (iii) Stable: } 62.7^{\mathrm{a}}\end{array}$ \\
\hline & & & $\begin{array}{l}\text { Overall liking on a } 100 \mathrm{~mm} \text { VAS; } \\
\text { Measured daily during the exposure period and } \\
\text { at pre- and post-exposure }\end{array}$ & $\begin{array}{l}\text { (i) Hazelnuts }(42 \mathrm{~g} / \mathrm{d}) \text {; Exp. period }=84 \mathrm{~d} \\
\text { (ii) Chocolate }(50 \mathrm{~g} / \mathrm{d}) \text {; Exp. period }=84 \mathrm{~d} \\
\text { (iii) Potato crisps }(50 \mathrm{~g} / \mathrm{d}) ; \text { Exp. period }=84 \mathrm{~d}\end{array}$ & $\begin{array}{c}\text { 84-day exposure period } \\
\text { (i) Stable: } 57.9^{\mathrm{a}} \\
\text { Pre- vs. Post- } \\
\text { (ii) Decrease over time: }-9.9^{\mathrm{a}}(p=0.002) \\
\text { (iii) Decrease over time: }-8.6^{\mathrm{a}}(p=0.031) \\
\text { (i) No significant change: } 61.1^{\mathrm{a}} \text { vs. } 53.8^{\mathrm{a}} \\
\text { (ii) Significant decrease: } 76.2^{\mathrm{a}} \text { vs. } 53.6^{\mathrm{a}}(p<0.001) \\
\text { (iii) No significant change: } 67.0^{\mathrm{a}} \text { vs. } 58.0^{\mathrm{a}}\end{array}$ \\
\hline \multirow[b]{2}{*}{ Tey et al., 2013 [47] } & \multirow[b]{2}{*}{$\begin{array}{l}\text { Randomised } \\
\text { Parallel } \\
3 \text { treatments }\end{array}$} & $\begin{array}{l}107(46 \mathrm{M}, 61 \mathrm{~F}) \\
\text { Overweight and } \\
\text { obese individuals } \\
\text { with a }\end{array}$ & $\begin{array}{l}\text { Desire to consume on a } 150 \mathrm{~mm} \text { VAS; Measured } \\
\text { daily during the exposure period }\end{array}$ & $\begin{array}{l}\text { (i) Hazelnuts }(30 \mathrm{~g} / \mathrm{d}) \text {; Exp. period }=84 \mathrm{~d} \\
\text { (ii) Hazelnuts }(60 \mathrm{~g} / \mathrm{d}) \text {; Exp. period }=84 \mathrm{~d}\end{array}$ & $\begin{array}{c}\text { 84-day exposure period } \\
\text { (i) Increase over time: }+14.2^{\mathrm{a}}(p=0.003) \\
\text { (ii) Decrease over time: }-29.4^{\mathrm{b}}(p<0.001)\end{array}$ \\
\hline & & $\mathrm{BMI} \geq 25 \mathrm{~kg} / \mathrm{m}^{2}$ & $\begin{array}{l}\text { Overall liking on a } 150 \mathrm{~mm} \text { VAS; } \\
\text { Measured daily during the exposure period and } \\
\text { at pre- and post-exposure }\end{array}$ & $\begin{array}{l}\text { (i) Hazelnuts }(30 \mathrm{~g} / \mathrm{d}) \text {; Exp. period }=84 \mathrm{~d} \\
\text { (ii) Hazelnuts }(60 \mathrm{~g} / \mathrm{d}) \text {; Exp. period }=84 \mathrm{~d}\end{array}$ & $\begin{array}{c}\text { (i) 84-day exposure period } \\
\text { (i) Stable: }+0.4^{\mathrm{a}} \\
\text { (ii) Decrease over time: }-24.4^{\mathrm{b}}(p<0.001) \\
\text { Pre- vs. Post- } \\
\text { (i) vs. (ii): }+14.6(p<0.05)\end{array}$ \\
\hline Tey et al., 2015 [59] & Single intervention & aged above 18 years & $\begin{array}{l}\text { Overall liking on a } 150 \mathrm{~mm} \text { VAS; Measured daily } \\
\text { during the exposure period }\end{array}$ & $\begin{array}{l}\text { (i) Māori: Hazelnuts }(30 \mathrm{~g} / \mathrm{d}), \text { Exp. period }=28 \mathrm{~d} \\
\text { (ii) European: Hazelnuts }(30 \mathrm{~g} / \mathrm{d}) \text {, Exp. period }=28 \mathrm{~d}\end{array}$ & $\begin{array}{l}\text { 28-day exposure period } \\
\text { (i) No significant change } \\
\text { (ii) No significant change } \\
\text { Pre- vs. Post- } \\
\text { (i) vs. (ii): No difference }\end{array}$ \\
\hline
\end{tabular}


Table 10. Cont.

\begin{tabular}{|c|c|c|c|c|c|}
\hline Author, Year & Study Design & Subjects & $\begin{array}{l}\text { Measurement; } \\
\text { Timepoint }\end{array}$ & Treatments; Number of Exposures & Results $^{1}$ \\
\hline \multirow[b]{2}{*}{ Tey et al., 2015 [48] } & \multirow{2}{*}{$\begin{array}{c}\text { Randomised } \\
\text { Crossover } \\
6 \text { treatments (only } 3 \\
\text { hazelnut treatments } \\
\text { reported) }\end{array}$} & \multirow{2}{*}{$\begin{array}{l}74(34 \mathrm{M}, 40 \mathrm{~F}) \\
\text { healthy participants }\end{array}$} & $\begin{array}{l}\text { Desire to consume on a } 150 \mathrm{~mm} \text { VAS; Measured } \\
\text { daily during the exposure period }\end{array}$ & $\begin{array}{l}\text { (i) Ground hazelnuts }(30 \mathrm{~g} / \mathrm{d}) \text {; Exp. period }=5 \mathrm{~d} \\
\text { (ii) Sliced hazelnuts }(30 \mathrm{~g} / \mathrm{d}) \text { Exp. period }=5 \mathrm{~d} \\
\text { (iii) Whole hazelnuts }(30 \mathrm{~g} / \mathrm{d}) ; \text { Exp. period }=5 \mathrm{~d}\end{array}$ & $\begin{array}{l}\text { 5-day exposure period } \\
\text { (i) Stable: } \mathrm{a} \\
\text { (ii) Stable: }{ }^{\mathrm{b}} \\
\text { (iii) Stable: }{ }^{\mathrm{c}}\end{array}$ \\
\hline & & & $\begin{array}{l}\text { Overall liking on a } 150 \mathrm{~mm} \text { VAS; } \\
\text { Measured daily during the exposure period and } \\
\text { at pre- and post-exposure }\end{array}$ & $\begin{array}{l}\text { (i) Ground hazelnuts }(30 \mathrm{~g} / \mathrm{d}) \text {; Exp. period }=5 \mathrm{~d} \\
\text { (ii) Sliced hazelnuts }(30 \mathrm{~g} / \mathrm{d}) \text {; Exp. period }=5 \mathrm{~d} \\
\text { (iii) Whole hazelnuts }(30 \mathrm{~g} / \mathrm{d}) \text {; Exp. period }=5 \mathrm{~d}\end{array}$ & $\begin{array}{l}\text { 5-day exposure period } \\
\text { (i) Stable: }{ }^{\mathrm{a}} \\
\text { (ii) Stable: }{ }^{\mathrm{b}} \\
\text { (iii) Stable: }^{\mathrm{c}}\end{array}$ \\
\hline \multirow[b]{2}{*}{ Tey et al., 2017 [49] } & \multirow[b]{2}{*}{$\begin{array}{l}\text { Randomised } \\
\text { Crossover } \\
2 \text { treatments }\end{array}$} & \multirow[b]{2}{*}{$\begin{array}{l}72(24 \mathrm{M}, 48 \mathrm{~F}) \\
\text { Aged } 18 \text { years } \\
\text { and above }\end{array}$} & $\begin{array}{l}\text { Desire to consume on a } 150 \mathrm{~mm} \text { VAS; Measured } \\
\text { daily during the exposure period }\end{array}$ & $\begin{array}{l}\text { (i) Raw hazelnuts }(30 \mathrm{~g} / \mathrm{d}) \text {; Exp. period }=28 \mathrm{~d} \\
\text { (ii) Dry roasted, lightly salted hazelnuts }(30 \mathrm{~g} / \mathrm{d}) \text {; } \\
\text { Exp. period }=28 \mathrm{~d}\end{array}$ & $\begin{array}{l}\text { 28-day exposure period } \\
\text { (i) Stable: }{ }^{a} \\
\text { (ii) Stable: }{ }^{\text {a }}\end{array}$ \\
\hline & & & $\begin{array}{l}\text { Overall liking on a } 150 \mathrm{~mm} \text { VAS; } \\
\text { Measured daily during the exposure period and } \\
\text { at pre- and post-exposure }\end{array}$ & $\begin{array}{l}\text { (i) Raw hazelnuts }(30 \mathrm{~g} / \mathrm{d}) \text {; Exp. period }=28 \mathrm{~d} \\
\text { (ii) Dry roasted, lightly salted hazelnuts }(30 \mathrm{~g} / \mathrm{d}) \text {; } \\
\text { Exp. period }=28 \mathrm{~d}\end{array}$ & $\begin{array}{l}\text { 28-day exposure period } \\
\text { (i) Stable: }{ }^{\mathrm{a}} \\
\text { (ii) Stable: }{ }^{\mathrm{a}} \\
\text { Pre- vs. Post- } \\
\text { (i) No significant change: } 105^{\mathrm{a}} \text { vs. } 108^{\mathrm{a}} \\
\text { (ii) No significant change: } 107^{\mathrm{a}} \text { vs. } 111^{\mathrm{a}}\end{array}$ \\
\hline
\end{tabular}

Abbreviations used: Exp., exposure; F, female; M, male; No., number; VAS, visual analogue scale. All values are arithmetic means unless otherwise stated. ${ }^{1}$ No acceptance results for no nut control group. Results: ${ }^{a}, \mathrm{~b}, \mathrm{c}$ Between-group comparisons, determined using ANOVA or regression models $(p<0.05)$. 


\section{Discussion}

Overall, we identified 22 studies (25 papers) that examined the effects of hazelnut consumption on at least one of the outcomes of interest. Many of the studies suffered from methodological flaws, including lack of randomisation, lack of a control group, small samples, short duration, lack of between-group analyses, and poor reporting of findings. These factors may account for some of the inconsistent findings. One finding that was consistent with previous literature on other nut types is the null effect on body weight. In addition, overall liking and desire to consume ratings remained stable over time, suggesting hazelnuts are resistant to monotony.

Only 9 of the 17 studies, which examined blood lipids and lipoproteins, reported between-group differences. Of these, only two studies $[57,58]$ reported significant reductions in total and LDL-C with hazelnut consumption. Four studies also reported significantly higher HDL-C concentrations with hazelnut consumption when compared to a non-nut control $[36,38,57]$ or a high carbohydrate diet [55]. Orem et al. also reported a significant increase in apo A [57]. Only one study reported a significant decrease in TAG [57]. The sample sizes for each treatment were small, ranging from 10 to 25 . This reduces the power to detect significant differences.

Two randomised crossover studies with larger samples ( $n=48$ to 72 ) compared different forms of hazelnuts and reported no significant differences in lipoprotein profiles. However, compared to baseline, hazelnut consumption significantly reduced total cholesterol, LDL-C, and apo B, and significantly increased HDL-C and apo A [43,49].

Several meta-analyses have reported significant improvements in blood lipids and apolipoprotein profiles with nut consumption [12-14]. The magnitude of the effect was greater among those with higher baseline concentrations and those with healthy body weight. There was also evidence of a dose-response relationship. In the present review, the majority of studies reported some improvement in at least one lipid parameter, with no studies reporting adverse effects. A meta-analysis of three RCTs found that hazelnutenriched diets were associated with a reduction in total cholesterol and LDL-C, with no changes in HDL-C or TAG [31]. This suggests that similar to other nut types, hazelnuts can be incorporated into a cardioprotective diet.

A total of 17 studies examined body composition, including body weight; BMI; waist, abdominal, and hip circumference; fat mass; and lean body mass. Except for one small single-intervention study among older adults, which showed a small but significant increase in body weight [56], the remainder of the studies among adults consistently reported no statistically or practically significant changes in body weight and composition as a result of adding hazelnuts to the diet. This is irrespective of study design, study population, study duration, and dose of hazelnuts. This was still apparent when there was no dietary advice to make substitutions. This is in agreement with a recent meta-analysis, which showed a nut-enriched diet did not result in weight gain either with or without instructions on dietary substitutions [19]. One study among children showed a time effect where there was an increase in both body weight and height. However, this did not differ between the hazelnut groups and the no-nut control. In two studies, favourable changes in body composition were seen among healthy participants $[52,53]$.

These findings are consistent with other studies, which have found no evidence of weight gain in the short-term following the addition of nuts to the diet $[11,19,61]$. In addition, a meta-analysis of three RCTs reported no change in body weight with hazelnut consumption [31]. Possible metabolic mechanisms for this lack of weight gain include higher metabolic rate due to the high unsaturated fat content of nuts, reduced lipid bioaccessibility and higher faecal losses of lipids due to the incomplete mastication and intact cell wall of whole nuts [62]. A further possible mechanism is increased satiety, which is influenced by a number of properties found in nuts, such as the fibre and protein content, and crunchy texture, which leads to increased oral exposure time and reduced post-prandial drive for food $[11,63]$. Our review agrees with previous research on different 
nut types $[11,19,24,64]$ and suggests that hazelnuts can be added to the diet without fear of adverse weight gain.

Seven studies measured blood pressure [39,47,49,50,53,56,59]. No significant changes to blood pressure were found as a result of adding hazelnuts to the diet. One study that included lightly salted nuts reported no significant differences in blood pressure when compared to raw, unsalted nuts. This is consistent with current literature where the effect of nut consumption on blood pressure remains equivocal, but there are suggestions of potential benefits in some sub-groups such as those with hypertension or among those without type 2 diabetes $[65,66]$. In addition, some nut types may be more effective, with a meta-analysis suggesting pistachios may be effective at reducing blood pressure $[26,66]$. The null finding is perhaps not unexpected, given the studies in the current review were conducted in relatively normotensive participants.

Nine studies measured some aspects of glycaemia, including fasting blood glucose, $\mathrm{HbA1c}$, post-prandial blood glucose, fasting insulin, postprandial insulin, HOMA-IR, and iAUC for blood glucose. Consuming hazelnuts as part of a carbohydrate-rich food resulted

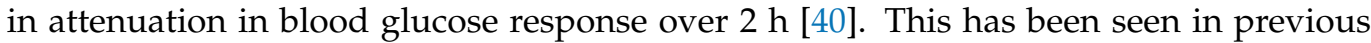
nut studies-including almonds $[67,68]$ among healthy populations and pistachios among people with metabolic syndrome [69].

Longer-term studies in individuals with normoglycaemia showed no practical benefits from hazelnut consumption on glycaemic control. However, a single intervention among people with type 2 diabetes reported a reduction in HbAlc over 30 days [51]. It should be noted that both studies, which included people with type 2 diabetes, showed no improvements in fasting blood glucose concentrations. The mixed results are consistent with studies examining different types of nuts. Several studies have shown a lack of positive effects on glycaemia for nuts, including walnuts, almonds, and cashews [25,30,70]. In agreement with Alphan et al., a meta-analysis suggested that there may be improvements in $\mathrm{HbA} 1 \mathrm{c}$ among people with diabetes. Collectively, the results from our review suggest that while the addition of hazelnuts to meals acutely attenuates glycaemic response, the long-term effects are less clear and require further investigation among healthy populations and those with type 2 diabetes.

Hazelnuts are rich in antioxidants [71-73]. Most of the studies reported increases in antioxidant status, but this was not consistently translated into improvements of biomarkers of oxidative stress. Studies assessed different biomarkers, had relatively small samples and used different study designs and analytical methods. Previous reviews have also produced heterogenous findings $[18,74]$, making it challenging to form definitive conclusions on the effects of nut consumption on oxidative stress.

Six of the seven studies which examined inflammation reported no improvements. This lack of change in inflammatory markers with nut consumption, in general, was seen in previous systematic reviews and meta-analyses [75,76]. A meta-analysis of inflammatory markers, which conducted sub-group analyses, suggested improvements were seen in studies where the duration was 12 weeks and greater [17].

Five studies assessed some form of endothelial function, with three reporting improvements in outcomes and two showing no effects. Most meta-analyses on biomarkers of endothelial function report no effects with nut consumption. Those which measure flow-mediated dilation (FMD) report more favourable outcomes [76,77], especially for walnuts $[16,29]$.

Overall, there was evidence that hazelnut consumption can improve some markers of cardiometabolic health. These beneficial effects are likely driven by the nutrient composition of hazelnuts. Several studies have reported improvements in diet quality with the addition of hazelnuts to the diet. There is evidence of higher intakes of unsaturated fat, fibre, vitamin E, potassium, and lower intakes of carbohydrate and sodium $[43,47,49,78]$.

In addition to assessing the health effects of nut consumption, it is equally important to examine the acceptability of nuts over time. This is because to exert their health benefits, nuts must be consumed regularly and in sufficient quantities. Only one group has assessed 
long-term acceptance for hazelnuts. Collectively, the hazelnuts studies have reported sustained acceptance up to 12 weeks with doses of 30 to $42 \mathrm{~g} / \mathrm{d}$. One study showed a dose of $60 \mathrm{~g} / \mathrm{d}$ resulted in a decline in liking with repeated consumption, a phenomenon known as monotony [47]. Future studies should assess acceptance over longer periods. Several studies examined different forms of hazelnuts, including whole, sliced, and ground, as well as raw versus dry roasted, lightly salted [40,44,48,49]. All forms of hazelnuts were resistant to monotony. A further study compared three popular energy-dense snack foods-hazelnuts, chocolate, and potato crisps. Ratings of overall liking remained stable over 12 weeks for hazelnuts but declined significantly for the other two snack foods [46]. Overall, these results suggest that dietary guidelines to consume one serving of nuts (30 to $42 \mathrm{~g}$ ) on a regular basis are achievable and sustainable. Given that different forms of hazelnuts were equally liked, we can recommend the inclusion of different forms of nuts based on individual preference. This provides increased choice for consumers, enhancing adherence to advice to consume nuts regularly as part of a cardioprotective diet.

Studies, which have estimated the impact of substituting nuts for less healthful foods, have shown large reductions in mortality from cardiovascular disease $[79,80]$. In addition, a recent study reported that the total annual costs of cardiometabolic disease related to a suboptimal diet were $\$ 301$ per person. Among the 10 dietary factors examined in this study, a low intake of nuts or seeds was found to impose the largest cardiometabolic disease economic burden at $\$ 81$ per person [81]. Therefore, a small gradual diet change has the potential to reduce the risk of chronic disease. It seems prudent for healthcare professionals to promote the intake of healthy food such as nuts as part of a cardioprotective diet [82].

\section{Conclusions}

This comprehensive systematic review has reported the effects of hazelnut consumption on a wide range of outcomes. The findings show some improvements in cardiometabolic risk factors, but limitations in study design make interpretation difficult. However, there was consistent evidence that the inclusion of hazelnuts into the diet did not adversely affect body weight and composition. In addition, acceptance of hazelnuts remained stable over time, suggesting nut consumption guidelines are achievable and sustainable. Overall, none of the studies reported evidence of adverse outcomes, and thus the balance of the research suggests the benefits of hazelnut consumption outweigh any potential negative effects. This was apparent among populations that included healthy participants, as well as those with hyperlipidaemia, type 2 diabetes, overweight, and obesity. Future studies should use more robust study designs, including larger sample sizes, careful selection of biomarkers, and appropriate control groups.

Supplementary Materials: The following supporting information can be downloaded at: https:// www.mdpi.com/article/10.3390/ijerph19052880/s1, Table S1: Search terms.

Author Contributions: Conceptualization, R.B. and S.L.T.; methodology, R.B., L.W. and S.L.T.; validation, R.B., L.W. and S.L.T.; investigation, R.B., L.W. and S.L.T.; writing-original draft preparation, R.B., L.W. and S.L.T.; writing-review and editing, R.B., L.W. and S.L.T. All authors have read and agreed to the published version of the manuscript.

Funding: This research received no external funding.

Institutional Review Board Statement: Not applicable.

Informed Consent Statement: Not applicable.

Data Availability Statement: Not applicable.

Conflicts of Interest: The authors declare no conflict of interest. 


\section{References}

1. Becerra-Tomás, N.; Paz-Graniel, I.; Kendall, C.W.C.; Kahleova, H.; Rahelić, D.; Sievenpiper, J.L.; Salas-Salvadó, J. Nut consumption and incidence of cardiovascular diseases and cardiovascular disease mortality: A meta-analysis of prospective cohort studies. Nutr. Rev. 2019, 77, 691-709. [CrossRef] [PubMed]

2. Ros, E.; Singh, A.; O'Keefe, J.H. Nuts: Natural pleiotropic nutraceuticals. Nutrients 2021, 13, 3269. [CrossRef] [PubMed]

3. Zhang, D.; Dai, C.; Zhou, L.; Li, Y.; Liu, K.; Deng, Y.-J.; Li, N.; Zheng, Y.; Hao, Q.; Yang, S.; et al. Meta-analysis of the association between nut consumption and the risks of cancer incidence and cancer-specific mortality. Aging 2020, 12, 10772-10794. [CrossRef]

4. $\quad$ Becerra-Tomás, N.; Paz-Graniel, I.; Hernández-Alonso, P.; Jenkins, D.J.A.; Kendall, C.W.C.; Sievenpiper, J.L.; Salas-Salvadó, J. Nut consumption and type 2 diabetes risk: A systematic review and meta-analysis of observational studies. Am. J. Clin. Nutr. 2021, 113, 960-971. [CrossRef] [PubMed]

5. Luo, C.; Zhang, Y.; Ding, Y.; Shan, Z.; Chen, S.; Yu, M.; Hu, F.B.; Liu, L. Nut consumption and risk of type 2 diabetes, cardiovascular disease, and all-cause mortality: A systematic review and meta-analysis. Am. J. Clin. Nutr. 2014, 100, 256-269. [CrossRef] [PubMed]

6. Wu, L.; Wang, Z.; Zhu, J.; Murad, A.L.; Prokop, L.J.; Murad, M.H. Nut consumption and risk of cancer and type 2 diabetes: A systematic review and meta-analysis. Nutr. Rev. 2015, 73, 409-425. [CrossRef] [PubMed]

7. Zhou, D.; Yu, H.; He, F.; Reilly, K.H.; Zhang, J.; Li, S.; Zhang, T.; Wang, B.; Ding, Y.; Xi, B. Nut consumption in relation to cardiovascular disease risk and type 2 diabetes: A systematic review and meta-analysis of prospective studies. Am. J. Clin. Nutr. 2014, 100, 270-277. [CrossRef]

8. Brown, R.; Tey, S.; Gray, A.; Chisholm, A.; Smith, C.; Fleming, E.; Parnell, W. Association of nut consumption with cardiometabolic risk factors in the 2008/2009 New Zealand Adult Nutrition Survey. Nutrients 2015, 7, 5351. [CrossRef]

9. O'Neil, C.E.; Keast, D.R.; Nicklas, T.A.; Fulgoni, V.L. Nut consumption is associated with decreased health risk factors for cardiovascular disease and metabolic syndrome in U.S. adults: NHANES 1999-2004. J. Am. Coll. Nutr. 2011, 30, 502-510. [CrossRef]

10. Bes-Rastrollo, M.; Sabate, J.; Gomez-Gracia, E.; Alonso, A.; Martinez, J.A.; Martinez-Gonzalez, M.A. Nut consumption and weight gain in a Mediterranean cohort: The SUN Study. Obesity 2007, 15, 107-116. [CrossRef]

11. Nishi, S.K.; Viguiliouk, E.; Blanco Mejia, S.; Kendall, C.W.C.; Bazinet, R.P.; Hanley, A.J.; Comelli, E.M.; Salas Salvadó, J.; Jenkins, D.J.A.; Sievenpiper, J.L. Are fatty nuts a weighty concern? A systematic review and meta-analysis and dose-response meta-regression of prospective cohorts and randomized controlled trials. Obes. Rev. 2021, 22, e13330. [CrossRef] [PubMed]

12. Altamimi, M.; Zidan, S.; Badrasawi, M. Effect of tree nuts consumption on serum lipid profile in hyperlipidemic individuals: A systematic review. Nutr. Metab. Insights 2020, 13, 1178638820926521. [CrossRef] [PubMed]

13. Del Gobbo, L.C.; Falk, M.C.; Feldman, R.; Lewis, K.; Mozaffarian, D. Effects of tree nuts on blood lipids, apolipoproteins, and blood pressure: Systematic review, meta-analysis, and dose-response of 61 controlled intervention trials. Am. J. Clin. Nutr. 2015, 102, 1347-1356. [CrossRef]

14. Sabaté, J.; Oda, K.; Ros, E. Nut consumption and blood lipid levels: A pooled analysis of 25 intervention trials. Arch. Intern. Med. 2010, 170, 821-827. [CrossRef]

15. Yu, Z.; Malik, V.S.; Keum, N.; Hu, F.B.; Giovannucci, E.L.; Stampfer, M.J.; Willett, W.C.; Fuchs, C.S.; Bao, Y. Associations between nut consumption and inflammatory biomarkers. Am. J. Clin. Nutr. 2016, 104, 722-728. [CrossRef]

16. Xiao, Y.; Huang, W.; Peng, C.; Zhang, J.; Wong, C.; Kim, J.H.; Yeoh, E.-k.; Su, X. Effect of nut consumption on vascular endothelial function: A systematic review and meta-analysis of randomized controlled trials. Clin. Nutr. 2018, 37, 831-839. [CrossRef]

17. Xiao, Y.; Xia, J.; Ke, Y.; Cheng, J.; Yuan, J.; Wu, S.; Lv, Z.; Huang, S.; Kim, J.H.; Wong, S.Y.-s.; et al. Effects of nut consumption on selected inflammatory markers: A systematic review and meta-analysis of randomized controlled trials. Nutrition 2018, 54, 129-143. [CrossRef] [PubMed]

18. Silveira, B.K.S.; da Silva, A.; Hermsdorff, H.H.M.; Bressan, J. Effect of chronic consumption of nuts on oxidative stress: A systematic review of clinical trials. Crit. Rev. Food Sci. Nutr. 2022, 62, 726-737. [CrossRef]

19. Guarneiri, L.L.; Cooper, J.A. Intake of nuts or nut products does not lead to weight gain, independent of dietary substitution instructions: A systematic review and meta-analysis of randomized trials. Adv. Nutr. 2021, 12, 384-401. [CrossRef]

20. Alasalvar, C.; Shahidi, F. Tree nuts: Composition, phytochemicals, and health effects: An overview. In Tree Nuts: Composition, Phytochemicals, and Health Effect; Alasalvar, C., Shahidi, F., Eds.; Taylor \& Francis Group: Boca Raton, FL, USA, 2008; pp. 1-10.

21. Di Nunzio, M. Hazelnuts as source of bioactive compounds and health value underestimated food. Curr. Res. Nutr. Food Sci. 2019, 7, 17-28. [CrossRef]

22. Pelvan, E.; Alasalvar, C.; Uzman, S. Effects of roasting on the antioxidant status and phenolic profiles of commercial Turkish hazelnut varieties (Corylus avellana L.). J. Agric. Food Chem. 2012, 60, 1218-1223. [CrossRef] [PubMed]

23. Pelvan, E.; Olgun, E.Ö.; Karadağ, A.; Alasalvar, C. Phenolic profiles and antioxidant activity of Turkish Tombul hazelnut samples (natural, roasted, and roasted hazelnut skin). Food Chem. 2018, 244, 102-108. [CrossRef] [PubMed]

24. Dreher, M.L. A comprehensive review of almond clinical trials on weight measures, metabolic health biomarkers and outcomes, and the gut microbiota. Nutrients 2021, 13, 1968. [CrossRef] [PubMed]

25. Jamshidi, S.; Moradi, Y.; Nameni, G.; Mohsenpour, M.A.; Vafa, M. Effects of cashew nut consumption on body composition and glycemic indices: A meta-analysis and systematic review of randomized controlled trials. Diabetes Metab. Syndr. 2021, 15, 605-613. [CrossRef] [PubMed] 
26. Asbaghi, O.; Hadi, A.; Campbell, M.S.; Venkatakrishnan, K.; Ghaedi, E. Effects of pistachios on anthropometric indices, inflammatory markers, endothelial function and blood pressure in adults: A systematic review and meta-analysis of randomised controlled trials. Br. J. Nutr. 2021, 126, 718-729. [CrossRef] [PubMed]

27. Fogacci, F.; Cicero, A.F.G.; Derosa, G.; Rizzo, M.; Veronesi, M.; Borghi, C. Effect of pistachio on brachial artery diameter and flow-mediated dilatation: A systematic review and meta-analysis of randomized, controlled-feeding clinical studies. Crit. Rev. Food Sci. Nutr. 2019, 59, 328-335. [CrossRef] [PubMed]

28. Malmir, H.; Larijani, B.; Esmaillzadeh, A. The effect of walnut consumption on cardiometabolic profiles of individuals with abnormal glucose homoeostasis: A systematic review and meta-analysis of clinical trials. Br. J. Nutr. 2021, 1-31. [CrossRef]

29. Mohammadi-Sartang, M.; Bellissimo, N.; Totosy de Zepetnek, J.O.; Bazyar, H.; Mahmoodi, M.; Mazloom, Z. Effects of walnuts consumption on vascular endothelial function in humans: A systematic review and meta-analysis of randomized controlled trials. Clin. Nutr. ESPEN 2018, 28, 52-58. [CrossRef] [PubMed]

30. Neale, E.P.; Guan, V.; Tapsell, L.C.; Probst, Y.C. Effect of walnut consumption on markers of blood glucose control: A systematic review and meta-analysis. Br. J. Nutr. 2020, 124, 641-653. [CrossRef] [PubMed]

31. Perna, S.; Giacosa, A.; Bonitta, G.; Bologna, C.; Isu, A.; Guido, D.; Rondanelli, M. Effects of hazelnut consumption on blood lipids and body weight: A systematic review and Bayesian meta-analysis. Nutrients 2016, 8, 747. [CrossRef]

32. Ouzzani, M.; Hammady, H.; Fedorowicz, Z.; Elmagarmid, A. Rayyan-a web and mobile app for systematic reviews. Syst. Rev. 2016, 5, 210. [CrossRef] [PubMed]

33. Page, M.J.; McKenzie, J.E.; Bossuyt, P.M.; Boutron, I.; Hoffmann, T.C.; Mulrow, C.D.; Shamseer, L.; Tetzlaff, J.M.; Akl, E.A.; Brennan, S.E.; et al. The PRISMA 2020 statement: An updated guideline for reporting systematic reviews. BMJ 2021, 372, n71. [CrossRef] [PubMed]

34. Sterne, J.A.C.; Savović, J.; Page, M.J.; Elbers, R.G.; Blencowe, N.S.; Boutron, I.; Cates, C.J.; Cheng, H.-Y.; Corbett, M.S.; Eldridge, S.M.; et al. RoB 2: A revised tool for assessing risk of bias in randomised trials. BMJ 2019, 366, 14898. [CrossRef] [PubMed]

35. Sterne, J.A.C.; Hernán, M.A.; Reeves, B.C.; Savović, J.; Berkman, N.D.; Viswanathan, M.; Henry, D.; Altman, D.G.; Ansari, M.T.; Boutron, I.; et al. ROBINS-I: A tool for assessing risk of bias in non-randomised studies of interventions. BMJ 2016, 355 , i4919. [CrossRef]

36. Adamo, M.; Labate, A.M.; Ferrulli, A.; Macrì, C.; Terruzzi, I.; Luzi, L. Effects of hazelnuts and cocoa on vascular reactivity in healthy subjects: A randomised study. Int. J. Food Sci. Nutr. 2018, 69, 223-234. [CrossRef]

37. Damavandi, R.; Eghtesadi, S.; Shidfar, F.; Heydari, I.; Foroushani, A. Effects of hazelnut consumption on fasting blood sugar, total antioxidant capacity, hs-CRP and paraoxonase-1 activity in patients with type 2 diabetes. Razi J. Med. Sci. 2012, 19, 40-50.

38. Damavandi, R.; Eghtesadi, S.; Shidfar, F.; Heydari, I.; Foroushani, A. Effects of hazelnuts consumption on fasting blood sugar and lipoproteins in patients with type 2 diabetes. J. Res. Med. Sci. Off. J. Isfahan Univ. Med. Sci. 2013, 18, 314-321.

39. Deon, V.; Del Bo, C.; Guaraldi, F.; Abello, F.; Belviso, S.; Porrini, M.; Riso, P.; Guardamagna, O. Effect of hazelnut on serum lipid profile and fatty acid composition of erythrocyte phospholipids in children and adolescents with primary hyperlipidemia: A randomized controlled trial. Clin. Nutr. 2018, 37, 1193-1201. [CrossRef]

40. Devi, A.; Chisholm, A.; Gray, A.; Tey, S.L.; Williamson-Poutama, D.; Cameron, S.L.; Brown, R.C. Nut-enriched bread is an effective and acceptable vehicle to improve regular nut consumption. Eur. J. Nutr. 2016, 55, 2281-2293. [CrossRef]

41. Di Renzo, L.; Merra, G.; Botta, R.; Gualtieri, P.; Manzo, A.; Perrone, M.A.; Mazza, M.; Cascapera, S.; De Lorenzo, A. Post-prandial effects of hazelnut-enriched high fat meal on LDL oxidative status, oxidative and inflammatory gene expression of healthy subjects: A randomized trial. Eur Rev Med Pharm. Sci. 2017, 21, 1610-1626.

42. Guaraldi, F.; Deon, V.; Del Bo, C.; Vendrame, S.; Porrini, M.; Riso, P.; Guardamagna, O. Effect of short-term hazelnut consumption on DNA damage and oxidized LDL in children and adolescents with primary hyperlipidemia: A randomized controlled trial. $J$. Nutr. Biochem. 2018, 57, 206-211. [CrossRef] [PubMed]

43. Tey, S.L.; Brown, R.C.; Chisholm, A.W.; Delahunty, C.M.; Gray, R.; Williams, S.M. Effects of different forms of hazelnuts on blood lipids and $\alpha$-tocopherol concentrations in mildly hypercholesterolemic individuals. Eur. J. Clin. Nutr. 2011, 65, 117-124. [CrossRef] [PubMed]

44. Tey, S.L.; Brown, R.; Chisholm, A.; Gray, A.; Williams, S.; Delahunty, C. Current guidelines for nut consumption are achievable and sustainable: A hazelnut intervention. Br. J. Nutr. 2011, 105, 1503-1511. [CrossRef] [PubMed]

45. Tey, S.L.; Brown, R.; Gray, A.; Chisholm, A.; Delahunty, C. Nuts improve diet quality compared to other energy-dense snacks while maintaining body weight. J. Nutr. Metab. 2011, 2011, 357350. [CrossRef] [PubMed]

46. Tey, S.L.; Brown, R.C.; Gray, A.R.; Chisholm, A.W.; Delahunty, C.M. Long-term consumption of high energy-dense snack foods on sensory-specific satiety and intake. Am. J. Clin. Nutr. 2012, 95, 1038-1047. [CrossRef]

47. Tey, S.L.; Gray, A.R.; Chisholm, A.W.; Delahunty, C.M.; Brown, R.C. The dose of hazelnuts influences acceptance and diet quality but not inflammatory markers and body composition in overweight and obese individuals. J. Nutr. 2013, 143, 1254-1262. [CrossRef]

48. Tey, S.L.; Delahunty, C.; Gray, A.; Chisholm, A.; Brown, R.C. Effects of regular consumption of different forms of almonds and hazelnuts on acceptance and blood lipids. Eur. J. Nutr. 2015, 54, 483-487. [CrossRef]

49. Tey, S.L.; Robinson, T.; Gray, A.R.; Chisholm, A.W.; Brown, R.C. Do dry roasting, lightly salting nuts affect their cardioprotective properties and acceptability? Eur. J. Nutr. 2017, 56, 1025-1036. [CrossRef] 
50. Yilmaz, H.Ö.; Özyildirim, B. Evaluation of the effects of raisins and hazelnuts added to the diet on lipid profiles and anthropometric measurements in women with hyperlipidemia. Bezmiâlem Sci. 2019, 7, 294-306. [CrossRef]

51. Alphan, E.; Pala, M.; Ackurt, F.; Yilmaz, T. Nutritional composition of hazelnuts and its effects on glucose and lipid metabolism. Acta Hort 1997, 445, 305-310. [CrossRef]

52. Di Renzo, L.; Alberto, C.; Daniela, M.; Botta, R.; Contessa, C.; Sartor, C.; Iacopino, A.M.; De Lorenzo, A. Nutrient Analysis Critical Control Point (NACCP): Hazelnut as a prototype of nutrigenomic study. Food Nutr. Sci. 2014, 5, 79-88. [CrossRef]

53. Di Renzo, L.; Cioccoloni, G.; Bernardini, S.; Abenavoli, L.; Aiello, V.; Marchetti, M.; Cammarano, A.; Alipourfard, I.; Ceravolo, I.; Gratteri, S. A hazelnut-enriched diet modulates oxidative stress and inflammation gene expression without weight gain. Oxidative Med. Cell. Longev. 2019, 2019, 4683723. [CrossRef] [PubMed]

54. Durak, I.; Köksal, I.; Kaçmaz, M.; Büyükkoçak, S.; Çimen, B.M.Y.; Öztürk, H.S. Hazelnut supplementation enhances plasma antioxidant potential and lowers plasma cholesterol levels. Clin. Chim. Acta 1999, 284, 113-115. [CrossRef]

55. Mercanligil, S.M.; Arslan, P.; Alasalvar, C.; Okut, E.; Akgul, E.; Pinar, A.; Geyik, P.O.; Tokgozoglu, L.; Shahidi, F. Effects of hazelnut-enriched diet on plasma cholesterol and lipoprotein profiles in hypercholesterolemic adult men. Eur. J. Clin. Nutr. 2007, 61, 212-220. [CrossRef]

56. Michels, A.J.; Leonard, S.W.; Uesugi, S.L.; Bobe, G.; Frei, B.; Traber, M.G. Daily consumption of Oregon hazelnuts affects $\alpha$-tocopherol status in healthy older adults: A pre-post intervention study. J. Nutr. 2018, 148, 1924-1930. [CrossRef] [PubMed]

57. Orem, A.; Yucesan, F.B.; Orem, C.; Akcan, B.; Kural, B.V.; Alasalvar, C.; Shahidi, F. Hazelnut-enriched diet improves cardiovascular risk biomarkers beyond a lipid-lowering effect in hypercholesterolemic subjects. J. Clin. Lipidol. 2013, 7, 123-131. [CrossRef]

58. Santi, C.; Giorni, A.; Terenzi, C.T.; Altavista, P.; Bacchetta, L. Daily hazelnut intake exerts multiple reversible effects on plasma profile of healthy subjects. Food Nutr. Sci. 2017, 8, 633-646. [CrossRef]

59. Tey, S.L.; Robinson, T.; Davis, H.; Gray, A.R.; Chisholm, A.W.; Brown, R.C. The effect of regular hazelnut consumption on cardiovascular risk factors and acceptance in Māori and European. J. Nutr. 2015, 2, 39-53. [CrossRef]

60. Yücesan, F.B.; Örem, A.; Kural, B.V.; Örem, C.; Turan, İ. Hazelnut consumption decreases the susceptibility of LDL to oxidation, plasma oxidized LDL level and increases the ratio of large/small LDL in normolipidemic healthy subjects. Anadolu Kardiyol Derg 2010, 10, 28-35. [CrossRef]

61. Akhlaghi, M.; Ghobadi, S.; Zare, M.; Foshati, S. Effect of nuts on energy intake, hunger, and fullness, a systematic review and meta-analysis of randomized clinical trials. Crit. Rev. Food Sci. Nutr. 2020, 60, 84-93. [CrossRef]

62. Franco Estrada, Y.M.; Caldas, A.P.S.; da Silva, A.; Bressan, J. Effects of acute and chronic nuts consumption on energy metabolism: A systematic review of randomised clinical trials. Int. J. Food Sci. Nutr. 2021, 1-11. [CrossRef] [PubMed]

63. Mattes, R.D.; Dreher, M.L. Nuts and healthy body weight maintenance mechanisms. Asia Pac. J. Clin. Nutr. 2010, 19, 137-141. [PubMed]

64. Fernández-Rodríguez, R.; Martínez-Vizcaíno, V.; Garrido-Miguel, M.; Martínez-Ortega, I.A.; Álvarez-Bueno, C.; Eumann Mesas, A. Nut consumption, body weight, and adiposity in patients with type 2 diabetes: A systematic review and meta-analysis of randomized controlled trials. Nutr. Rev. 2021. [CrossRef] [PubMed]

65. Barbour, J.A.; Howe, P.R.C.; Buckley, J.D.; Bryan, J.; Coates, A.M. Nut consumption for vascular health and cognitive function. Nutr. Res. Rev. 2014, 27, 131-158. [CrossRef]

66. Mohammadifard, N.; Salehi-Abargouei, A.; Salas-Salvadó, J.; Guasch-Ferré, M.; Humphries, K.; Sarrafzadegan, N. The effect of tree nut, peanut, and soy nut consumption on blood pressure: A systematic review and meta-analysis of randomized controlled clinical trials. Am. J. Clin. Nutr. 2015, 101, 966-982. [CrossRef]

67. Jenkins, D.J.; Kendall, C.W.; Josse, A.R.; Salvatore, S.; Brighenti, F.; Augustin, L.S.; Ellis, P.R.; Vidgen, E.; Rao, A.V. Almonds decrease postprandial glycemia, insulinemia, and oxidative damage in healthy individuals. J. Nutr. 2006, 136, $2987-2992$. [CrossRef]

68. Josse, A.R.; Kendall, C.W.; Augustin, L.S.; Ellis, P.R.; Jenkins, D.J. Almonds and postprandial glycemia-a dose-response study. Metabolism 2007, 56, 400-404. [CrossRef]

69. Kendall, C.W.C.; West, S.G.; Augustin, L.S.; Esfahani, A.; Vidgen, E.; Bashyam, B.; Sauder, K.A.; Campbell, J.; Chiavaroli, L.; Jenkins, A.L.; et al. Acute effects of pistachio consumption on glucose and insulin, satiety hormones and endothelial function in the metabolic syndrome. Eur. J. Clin. Nutr. 2014, 68, 370-375. [CrossRef]

70. Tindall, A.M.; Johnston, E.A.; Kris-Etherton, P.M.; Petersen, K.S. The effect of nuts on markers of glycemic control: A systematic review and meta-analysis of randomized controlled trials. Am. J. Clin. Nutr. 2019, 109, 297-314. [CrossRef]

71. Alasalvar, C.; Bolling, B.W. Review of nut phytochemicals, fat-soluble bioactives, antioxidant components and health effects. Br. J. Nutr. 2015, 113, S68-S78. [CrossRef]

72. Alasalvar, C.; Salvadó, J.-S.; Ros, E. Bioactives and health benefits of nuts and dried fruits. Food Chem. 2020, 314, 126192. [CrossRef] [PubMed]

73. Bolling, B.W.; Chen, C.Y.; McKay, D.L.; Blumberg, J.B. Tree nut phytochemicals: Composition, antioxidant capacity, bioactivity, impact factors. A systematic review of almonds, Brazils, cashews, hazelnuts, macadamias, pecans, pine nuts, pistachios and walnuts. Nutr. Res. Rev. 2011, 24, 244-275. [CrossRef] [PubMed]

74. López-Uriarte, P.; Bulló, M.; Casas-Agustench, P.; Babio, N.; Salas-Salvadó, J. Nuts and oxidation: A systematic review. Nutr. Rev. 2009, 67, 497-508. [CrossRef] [PubMed] 
75. Mazidi, M.; Rezaie, P.; Ferns, G.A.; Gao, H.-k. Impact of different types of tree nut, peanut, and soy nut consumption on serum C-reactive protein (CRP): A systematic review and meta-analysis of randomized controlled clinical trials. Medicine 2016, 95 , e5165. [CrossRef]

76. Neale, E.P.; Tapsell, L.C.; Guan, V.; Batterham, M.J. The effect of nut consumption on markers of inflammation and endothelial function: A systematic review and meta-analysis of randomised controlled trials. BMJ Open 2017, 7, e016863. [CrossRef] [PubMed]

77. Smeets, E.T.H.C.; Mensink, R.P.; Joris, P.J. Effects of tree nut and groundnut consumption compared with those of 1-arginine supplementation on fasting and postprandial flow-mediated vasodilation: Meta-analysis of human randomized controlled trials. Clin. Nutr. 2021, 40, 1699-1710. [CrossRef]

78. Pearson, K.R.; Tey, S.L.; Gray, A.R.; Chisholm, A.; Brown, R.C. Energy compensation and nutrient displacement following regular consumption of hazelnuts and other energy-dense snack foods in non-obese individuals. Eur. J. Nutr. 2017, 56, $1255-1267$. [CrossRef]

79. Lloyd-Williams, F.; Mwatsama, M.; Ireland, R.; Capewell, S. Small changes in snacking behaviour: The potential impact on CVD mortality. Public Health Nutr. 2009, 12, 871-876. [CrossRef]

80. Mozaffarian, D.; Capewell, S. United Nations' dietary policies to prevent cardiovascular disease. BMJ 2011, 343, d5747. [CrossRef]

81. Jardim, T.V.; Mozaffarian, D.; Abrahams-Gessel, S.; Sy, S.; Lee, Y.; Liu, J.; Huang, Y.; Rehm, C.; Wilde, P.; Micha, R.; et al. Cardiometabolic disease costs associated with suboptimal diet in the United States: A cost analysis based on a microsimulation model. PLoS Med. 2019, 16, e1002981. [CrossRef]

82. Mozaffarian, D.; Appel, L.J.; Horn, L.V. Components of a cardioprotective diet. Circulation 2011, 123, 2870-2891. [CrossRef] [PubMed] 